\title{
2006 Status of the Momentum eXchange Electrodynamic Re-boost (MXER) Tether Development
}

\author{
Joseph A. Bonometti ${ }^{*}$ and Kirk F. Sorensen ${ }^{\dagger}$ \\ NASA - Marshall Space Flight Center, Huntsville, AL 35812 \\ John W. Dankanich ${ }^{\ddagger}$ and Kyle L. Frame ${ }^{\S}$ \\ Gray Research, Inc., Huntsville, AL, 35806
}

\begin{abstract}
The MXER Tether technology development is a high-payoff/high-risk investment area within the NASA In-Space Propulsion Technology (ISPT) Program. The ISPT program is managed by the NASA Headquarters Science Mission Directorate and implemented by the Marshall Space Flight Center in Huntsville, Alabama. The MXER concept was identified and competitively ranked within NASA's comprehensive Integrated In-Space Transportation Plan (IISTP) ${ }^{1}$; an agency-wide technology assessment activity. The objective of the MXER tether project within ISPT is to advance the technological maturation level for the MXER system, and its subsystems, as well as other space and terrestrial tether applications. Recent hardware efforts have focused on the manufacturability of spacesurvivable high-strength tether material and coatings, high-current electrodynamic tether, lightweight catch mechanism, high-accuracy propagator/predictor code, and efficient electron collection/current generation. Significant technical progress has been achieved with modest ISPT funding to the extent that MXER has evolved to a well-characterized system with greater capability as the design has been matured. Synergistic efforts in high-current electrodynamic tethers and efficient electron collection/current generation have been made possible through SBIR and STTR support. The entire development endeavor was orchestrated as a collaborative team effort across multiple individual contracts and has established a solid technology resource base, which permits a wide variety of future space cable/tether applications to be realized.
\end{abstract}

\section{Introduction}

M omentum-eXchange Electrodynamic Reboost (MXER) Tethers were derived from a combination of tether concepts including both Momentum eXchange (MX) and ElectroDynamic (ED) designs. A MX tether is a long thin cable or stringer used to couple two objects in space together so that one transfers momentum and orbital energy to the other. ${ }^{2}$ Many ideas for MX tether uses have been formulated through the years, but few have materialized. ${ }^{3}$ If only used once, an MX tether normally is not an effective use of "propulsion mass", having an equivalent specific impulse $\left(\mathrm{I}_{\mathrm{sp}}\right)$ on the order of 50 seconds. Reusable $\mathrm{MX}$ tethers require some type of reboost propulsion due to the fact that orbital altitude (and thus the MX tether's energy and momentum) is "consumed". An ED tether is made of conductive material in order to carry electrical current. Electrical current flowing in the presence of the Earth's magnetic field causes a Lorentz force between the tether and the Earth that results in momentum-exchange-essentially the ED tether "pushes" against the Earth via the magnetic field. This is the same physical principle that causes any electric motor to turn. As such, ED tether propulsion is (ideally) a propellantless space propulsion system that utilizes only electrical power. However, the classical operational range has been limited to regions above the Earth where free electrons are available from the ionosphere (a region approximately $100-\mathrm{km}$ to $1000-\mathrm{km}$ above Earth) to be collected at one end of the 'wire' and then emitted at the other. Thus, the current in the conductor is only in one direction and a net body force is generated along the wire.

\footnotetext{
*Emerging Propulsion Technologies Manager, In-Space Propulsion Technology Project, VP51, Joseph.A.Bonometti@nasa.gov, Member, AIAA.

${ }^{\dagger}$ Lead Systems Engineer, In-Space Propulsion Technology Office, EI61, Kirk.F.Sorensen@̂nasa.gov, Member, AIAA.

$\ddagger$ Aerospace Engineer, In-Space Propulsion, John.Dankanich@nasa.gov, Member, AIAA.

${ }^{\S}$ Program Integration, In-Space Propulsion, Kyle.L.Frame@nasa.gov, Member, AIAA.
} 
Consequently, a reusable MX tether coupled with an ED tether could provide significance orbital energy to payloads in low-Earth orbit (LEO) without consuming propellant. The inspiration for a MXER tether came through a study contract for a "Cislunar Tether Transportation System" from the NASA Institute of Advanced Concepts (NIAC) in 1996. ${ }^{4}$ The contract was won by Dr. Robert Forward and Dr. Robert Hoyt of Tethers Unlimited. The original concept included two tethers (or skyhooks) in orbit about the Earth and a third tether orbiting the moon (the Lunar Rotovator). The payload would be launched from Earth onto a suborbital trajectory and be caught by the first hypersonic skyhook. The payload would then be thrown by the skyhook into a highly elliptical orbit that would intersect with the second skyhook and be thrown towards the Lunar Rotovator. The payload would rendezvous with the tip of the Lunar Rotovator and be set down onto the lunar surface. The tip picks up a return payload mass, and the process is simply repeated in the reverse direction. As the concept was refined by Forward and Hoyt, they eliminated a hypersonic catch by catching payloads from low-Earth orbit instead of from a suborbital trajectory. The apparently difficult orbital catch of the payload led them to investigate the opportunities of attempting to catch the payload again should the first catch attempt fail. This led them to baseline an elliptical equatorial orbit for the tether and circular equatorial orbit for the payload so that multiple catch opportunities would be feasible. With this "one-way" architecture, the tether would need to reboost itself, and Hoyt conceptualized the fusion of electrodynamic and momentum-exchange tethers into a design he called HEFT (High-Strength Electrodynamic Force Tether), which later was tagged the now familiar MXER Tether facility by Kirk Sorensen. ${ }^{5}$ The MXER Tether facility, shown in Figure 1, could be used for one-way traffic, such as geosynchronous communications satellites and interplanetary spacecraft.

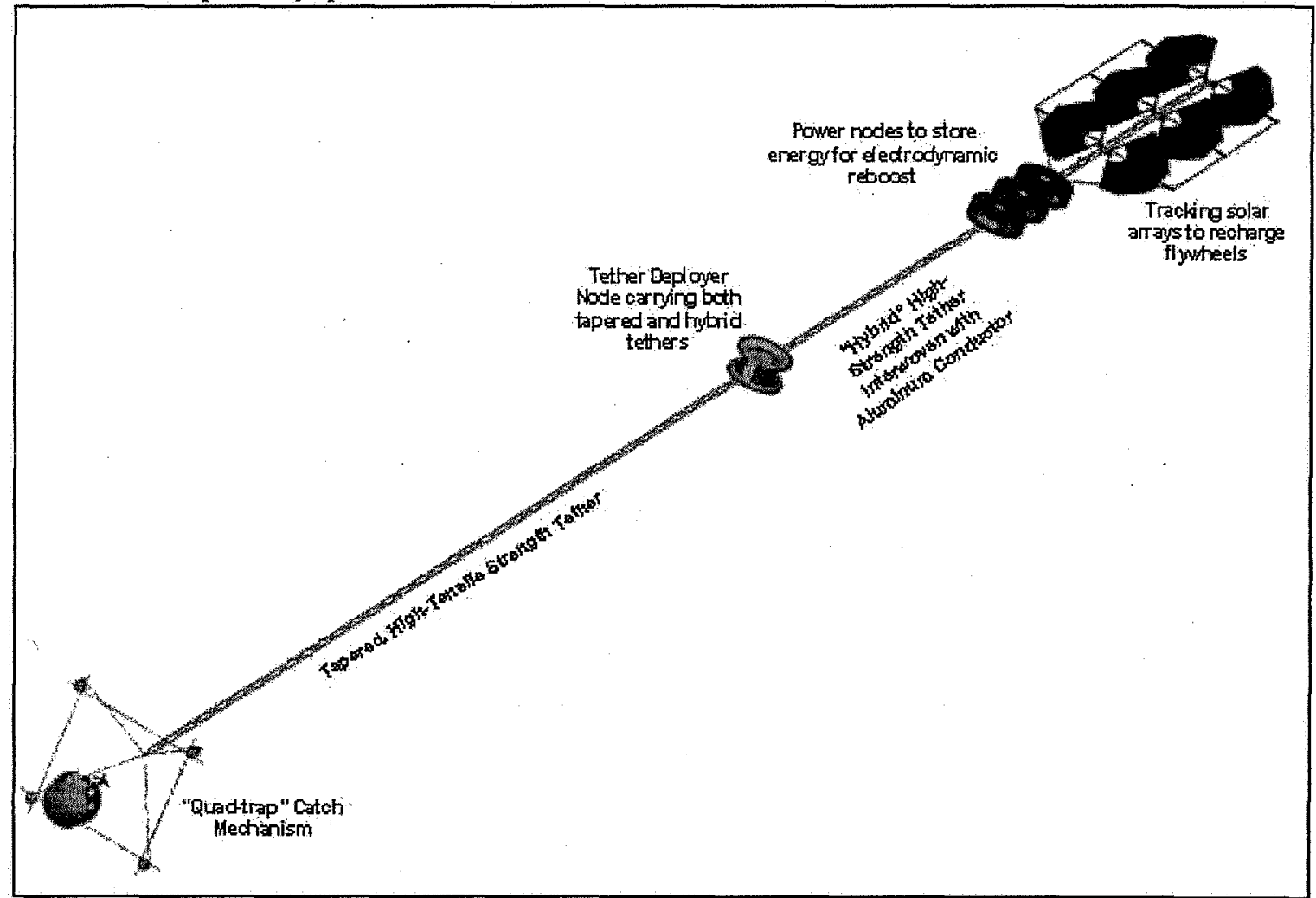

Figure 1: Conceptual MXER Tether Facility

In 2001, the NASA In-Space Propulsion Technology (ISPT) Program was created by the Science Mission Directorate (SMD), to develop the agency's next in-space propulsion technologies. SMD stipulated the numerous candidate propulsion technologies under the program be prioritized and categorized. This led to a process called the Integrated In-Space Transportation Plan (IISTP). IISTP considered a number of future SMD missions and each transportation technology's applicability to them. For the first time in such a competitive investigation, MXER tether transportation was considered. Throughout the mission set analyses, the MXER concept scored very high and had broad applicability, mostly due to the fact that the missions predominantly were medium sized interplanetary probes. Despite the objective methodology and rigorous evaluation rules imposed at the beginning of the IISTP 
process (including wrapping up the technical maturity into the judgment criterion), the raw analysis results were still subject to programmatic implementation decisions. MXER and two other propulsion concepts were removed from what would have been otherwise high-priority rankings due to their relatively low TRL. Based on the experience of the technologists assessing the data, a new category called, "High Payoff/High Risk" was created to capture the technologies that offered such high payoff but carried commensurately high risk due to their relative immaturity. This new category was to be managed by a newly created ISPT organization, Emerging Propulsion Technologies (EPT).

The first meeting of the MXER Tether Technology Assessment Group (TAG) was convened at the STAIF conference in Albuquerque, New Mexico, in February 2002. The assembled participants were briefed on the concepts and principles of the MXER tether system and, collectively, made recommendations as to which technologies merited the most attention under the first round of National Research Announcements (NRAs) that would be issued by the ISPT program. The TAG judged that the rendezvous and capture of the payload at the tether tip was a key technology that needed further research and development, and recommended that work be initiated in the fields of computational orbital analysis and propagation, and tether/payload catch mechanism development. They made further recommendations that a high-strength, survivable tether be developed and tested. A number of electrodynamic tether technologies were also analyzed, and while deemed important, their higher state of technical maturity due to NASA's TSS and ProSEDS efforts led to their development being deemed secondary to the MX tether technology development. Two key research areas not highlighted were the MXER power system as it relates to the overall system design trades and the hybrid or integrated conducting-strength tether. In both cases, these technologies were considered follow-on, or adaptations of the other components, and not driving system parameters.

Based on the recommendations of the TAG, a solicitation for MXER tether technology development was made under the ROSS Cycle II NRA for In-Space Propulsion. A number of proposals were received and evaluated by an independent (i.e., non-NASA) panel of experts, who recommended that the following awards be made subject to negotiation of terms:

- Tethers Unlimited Inc. (TUI) for computational propagator development and high-strength survivable tether.

- Lockheed Martin Space Systems (LMSS) for high-strength survivable tether and catch mechanism development.

- Tennessee Technological University (TTU) for catch mechanism development.

- Smithsonian Astrophysical Observatory (SAO) for computational propagator development.

The contracts with TUI, LMSS, and TTU were awarded in the timeframe from October 2003 to May 2004. Negotiations with SAO for computational propagator development were unsuccessful and the award was then given to the next ranked proposal. Star Technology and Research, Inc. (STAR) successfully negotiated its bid and began work in August 2004. These four organizations have been developing the majority of MXER tether technologies over the past four years. Coupled with Small Business Innovative Research (SBIR) and Small Business Technology Transfer (STTR) Program awards in the areas applicable to MXER tethers, the advancement in tethers, and specifically MXER tethers, has been remarkably fruitful. Not only are the technologies being developed useful for an assortment of transportation tethers, but other applications include solar panel tracking, thruster gimballing, military surveillance, large space asset station keeping, improved rendezvous techniques, flywheel power systems, instrument/communications pointing, and other innovative uses.

\section{Recent Tether Development at NASA}

As a result of the ROSS Cycle II NRA, recent efforts at NASA have gained a wealth of knowledge in the following areas within MXER tethers: computational propagator code, high-strength survivable tether, and the catch (and release) mechanism. By recommendation of the TAG, the computational propagator development was originally deemed as the high priority and, consequently, was planned to have the most funding. However, due to another TAG held in July 2003 and an independent assessment by the new EPT manager, a decision was made to fund the high-strength tether development as high priority with computational propagator development second priority and the catch mechanism low priority within this solicitation. Bridging the technology gaps associated with power systems (flywheels) and the hybrid tether was to be met later with limited funding from other sources. The EPT area has managed six NRA awards that are focused on the development of MXER. Four awards were planned, but funds for two supplementary proposals were available, allowing the top two proposals in each category to be selected. The contracts were all structured to be 'complementary' instead of 'competitive', so that the maximum research could be accomplished in the shortest time. This non-traditional approach, where all data is shared and 
none was company proprietary, allowed large trade spaces to be rapidly considered, the best ideas identified and the best suited individuals /teams to concentrate on priorities.

Additional MXER work has been performed within the Small Business Innovative Research (SBIR) and Small Business Technology Transfer (STTR) programs in the areas of strength tether manufacturing, plasma contactors, conductive tether/coatings development, and derivative technologies applicable to MXER including deployer mechanisms. The In-Space Propulsion Technology Project was designated the technical lead on four Phase II SBIRs and two Phase II STTRs related to MXER. These contracts were awarded in the late 2003/early 2004 timeframe, and all will be completed by late 2006 . These programs were developed to provide businesses with commercial opportunities; consequently, the information developed under these programs is proprietary. Therefore detailed information cannot be included in this paper, but may be requested directly from the individual companies.

\section{A. Strength Tether Development}

The current MXER design is a nominal $100 \mathrm{~km}$ spinning-tether composed of material with high specific tensile strength able to withstand a $2 \mathrm{~g}$ tip acceleration with payload attached. The tether, being in a harsh space environment, must be coated or treated in a manner to protect against atomic oxygen (AO) erosion and ultraviolet (UV) radiation which embrittles the high-strength polymers. The consideration of the coating's optical properties (i.e., absorptivity and emissivity), its wear and handling characteristics, conductivity (both thermal and electrical) and its mass impact are also extremely important. Furthermore, the envisioned MXER environment is heavily populated with micrometeoroid and orbital debris (MM/OD). Consequently, the tether is in a multi-strand, crosslinked configuration that provides redundant load paths in the event a strand is severed by MM/OD. The tether facility would also contain conductive tether portions $(\sim 10 \mathrm{~km}$ to $50 \mathrm{~km})$ that would provide electrodynamic reboost capability. The focus of the strength tether development under the Cycle II NRA converged on the multi-strand tether manufacturing process (yarn plying/twisting, bobbin winding, braiding, braider splicing, spooling, and quality control) along with the coating and/or treatment process for protection of $\mathrm{AO}$ and $\mathrm{UV} .{ }^{6}{ }^{7}$ A secondary goal of this effort was to begin the investigation into the development of the hybrid portion of the tether, the segment containing both conductive and strength members. This is a difficult challenge going beyond simple load path crosslinks, but embraces conductive interlinking and circuit protection following exposure of a conductor to the plasma environment.

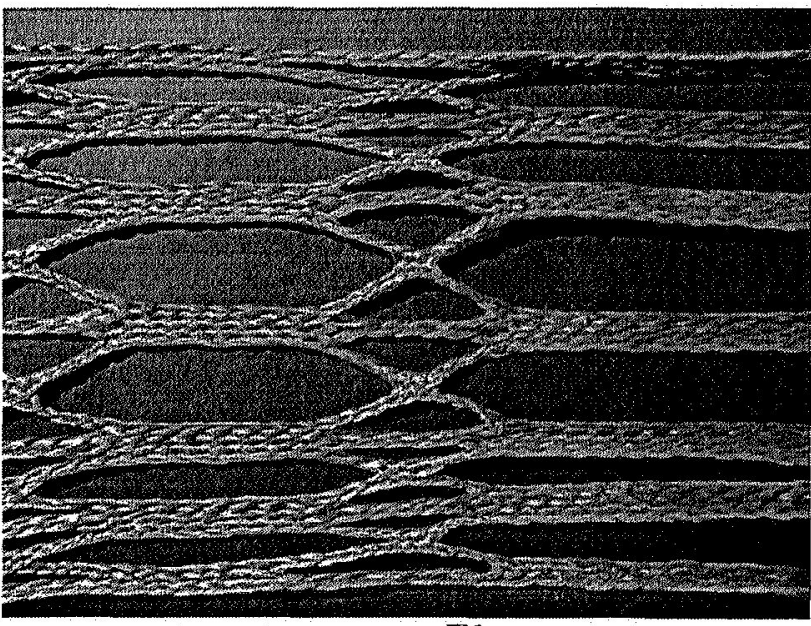

Figure 2: Hoytether ${ }^{\mathrm{TM}}$ Close-up

The objective of strength tether development, a combined effort with data shared between TUI and LMSS, was to develop a high specific strength multi-strand tether with atomic oxygen (AO) and ultraviolet radiation (UV) resistive coatings. ${ }^{8}$ TUI's Hoytether ${ }^{\mathrm{TM}}$, a multi-strand configuration shown in Figure 2 , reduces the risk of a tether sever from micrometeoroid debris. The tether is manufactured or braided in continuous fashion much as an intricate lace fabric is produced. The braiding technology was advanced, with emphasis on the relevant factors that affect the structure's strength, deployment and survivability. TUI made considerable progress in the process for manufacturing a Hoytether ${ }^{\mathrm{TM}}$ including automating the process of yarn twisting, transfer of yarn to braider bobbins, braiding the tether from the bobbins, and spooling the braided tether while establishing quality control checks throughout the manufacturing process. They aimed at complete automation, so that kilometer lengths can be easily manufactured in the future. Break strength tests demonstrated the structure snapped beyond the ideal material loading, thus ensuring that the interwoven connections were stronger than the base material and that the secondary cross-strapped strands began to be in tension. While TUI's Hoytether ${ }^{\mathrm{TM}}$ is considered the best tether configuration for MXER and many tether applications, LMSS did pursue an alternative laminated sheet configuration as a backup. ${ }^{9}$ Although the latter has the advantage of being simpler to package and deploy, its mass penalty was significant and the shearing load transfer between the primary lines was not conclusively demonstrated. Considering that TUI had no issues with winding/unwinding the woven tether structure, the laminated approach appears to have only secondary advantages for space applications, although it did provide excellent $\mathrm{AO} / \mathrm{UV}$ protection. 
Remarkable progress was made in coating the tethers for resistance against AO/UV. Both TUI and LMSS collaborated with Triton Systems Inc. (TSI) to use the TOR coating. Investigations were made into the process of wrapping TSI's TOR ${ }^{\mathrm{TM}}$ thread over PBO, or Zylon $\otimes$ yarn to provide an AO/UV resistant high-tenacity yarn with low mass impact and evaluate thin multi-dip TOR coatings on Zylon® yarns for atomic oxygen protection to

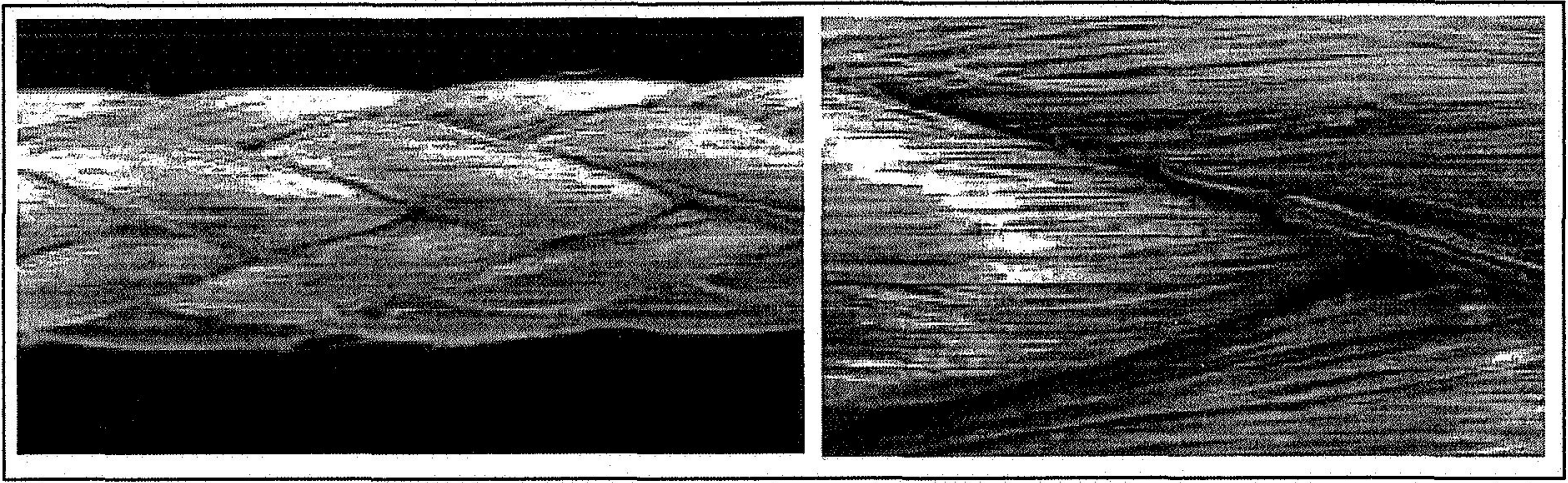

Figure 3: Lockheed Aluminum/alumina Coated Tether

improve flexibility \& adhesion. TUI and the Air Force Research Laboratory (AFRL) partnered to investigate the feasibility of protecting high-strength polymers such as Zylon $(B$ and Spectra@ from atomic oxygen degradation using thin coatings of $\mathrm{AO} / \mathrm{UV}$ resistant materials. This effort focused on incorporating polyhedral oligomeric silsesquioxane (POSS) into polymers compatible with the strength materials, and developing methods for applying thin coatings onto the tether yarns. TUI also joined forces with Integrity Testing Laboratories, who developed the Photosil ${ }^{\mathrm{TM}}$ coating, which incorporates silicon-containing functional groups into the top micron of an organic material. Zylon $®$ and Spectra $\circledast$ tether samples with Photosi ${ }^{\mathrm{TM}}$ were produced \& tested. Additionally, both TUI and LMSS metallized Zylon $\circledast$ fibers. TUI used nickel as the coating element while LMSS investigated multiple coatings with aluminum/alumina $\left(\mathrm{Al}^{2} \mathrm{O}^{3}\right)$, which displayed promise in $\mathrm{AO}$ protection when tested in the LMSS asher chamber. Lengths of Zylon (8) braids were protected by Al overcoated with aluminum oxide (Figure 3), which was deposited by a Plasma Vapor Deposition (PVD) process. The nickel coating was deposited in a multi-step process developed by Tethers Unlimited. LMSS also developed a "flat" tether that was a Zylon® braid encapsulated within bicomponent Mylar/polyethylene facesheets. These facesheets were coated both with $\mathrm{Al}$ and $\mathrm{Al} / \mathrm{Al}^{2} \mathrm{O}^{3}$ by PVD.

A preliminary investigation was made into the hybrid Hoytether ${ }^{\mathrm{TM}}$ portion of the MXER tether. TUI developed three new braiding methods (one for insulated wires and two for bare wire conductors) for fabrication of an integrated tether. TUI also began an initial investigation into NextGen self-healing wire insulation technologies

LMSS implemented a ranking criterion with their aluminum/alumina coating scoring well due to its lightweight, stowage/deployment flexibility, and interconnection enabling load transfer in the event of MM/OD impact, increasing survivability. However, issues remain to be addressed before full-scale demonstration, these include:

- Attaining full coating coverage of the entire braid structure (PVD coating process is inherently limited by line-of-sight restrictions imposed by the sputtering process)

- Analysis of coating abrasion when wrapped for stowage and/or deployment

LMSS also performed a literature search to investigate all the possible coatings and/or alternate tether material sources for space-survivable tethers. The report showed nothing unexpected, and the strength tether research in coatings and/or alternate tether material is on track for a smooth hand-off to a new NASA materials development program led by Auburn University. The coating direction to resist AO/UV still appears to be a thin aluminum base directly on the polymer (most likely M5) with an alumina coating for thermal control and electrical insulation.

TUI also advanced the manufacturing of multi-strand tethers under an STTR task that involved the planning of a flight experiment called the Multi-Application Survivable Tether (MAST) Experiment. ${ }^{10}{ }^{11}$ MAST's goal is to obtain on-orbit data on tether survivability while in the micrometeorite/orbital (M/OD) debris environment. The MAST experiment will deploy three $1-\mathrm{kg}$ CubeSats along a $1-\mathrm{km}$ Hoytether ${ }^{\mathrm{TM}}$. The middle CubeSat will then slowly translate along the tether, inspecting the cable as it moves and returning data on the rate of damage to the tether by M/OD impacts. The MAST experiment is set for launch in August, 2006, into $700 \mathrm{~km}$ orbit.

\section{B. Strength Tether Testing}

Samples from both TUI and LMSS were delivered to the Marshall Space Flight Center (MSFC) Atomic Oxygen Beam Facility (AOBF) and prepared for testing in an AO/UV environment. ${ }^{12}{ }^{13}$ Samples ranged from fifteen to 
twenty centimeters in length, depending on material availability. Samples were knotted twice before cutting to prevent loss of any of the fibers. To ensure that none of the materials were hygroscopic, each sample was placed into a vacuum chamber at 50 millitorr then immediately removed and weighed with readings being taken every minute for five minutes. Regression analysis to time zero was then used to eliminate any water weight gain. The samples were photographed before and after exposure to the simulated space environment. After simulated space exposure, maximum of approximately $3 \times 10^{21}$ atoms $/ \mathrm{cm}^{2}$, the samples were weighed for mass loss and mechanically tested to determine the tensile strength. Two rounds of testing were performed with TUI tether samples in the first round and LMSS samples in the second.

TUI's tether samples included both Spectra® and Zylon ${ }^{\circledR}$ coated with Photosil ${ }^{\mathrm{TM}}$, Zylon® strand metallized with nickel, Zylon(B) coated with TSI's TOR-LM ${ }^{\mathrm{TM}}$, Zylon® fiber coated POSS ${ }^{\mathrm{TM}}$, and uncoated M5 fiber. The Photosil ${ }^{\mathrm{TM}}$ and TOR-LM ${ }^{\mathrm{TM}}$ coatings did not provide the amount of protection that they had in previous experiments. According to the reactivity calculations, nickel coated Zylon® was able to resist AO's erosive effects, but due to it being a loose set of strands, tensile test results were scattered. Both the Spectra and Zylon $\circledast$ tether materials coated with Photosil ${ }^{\mathrm{TM}}$ lost tensile strength even without exposure to $\mathrm{AO}$ and $\mathrm{UV}$, which may be due to the process in which samples are prepared before receiving the Photosil ${ }^{\mathrm{TM}}$ coating. The nickel coating appeared to improve the Zylon(B) strand samples' resistance to $\mathrm{AO}$ erosion. Two nickel coated samples actually experienced mass gain by the end of their test regime suggesting that a nickel oxide had formed and was protecting the Zylon® from any further damage. The tensile test results were inconclusive, due to the handling difficulty with the strands. With visual observation, the TOR-LM ${ }^{\mathrm{TM}}$ coated Zylon $($ appeared to provide some protection from $\mathrm{AO}$ as compared to

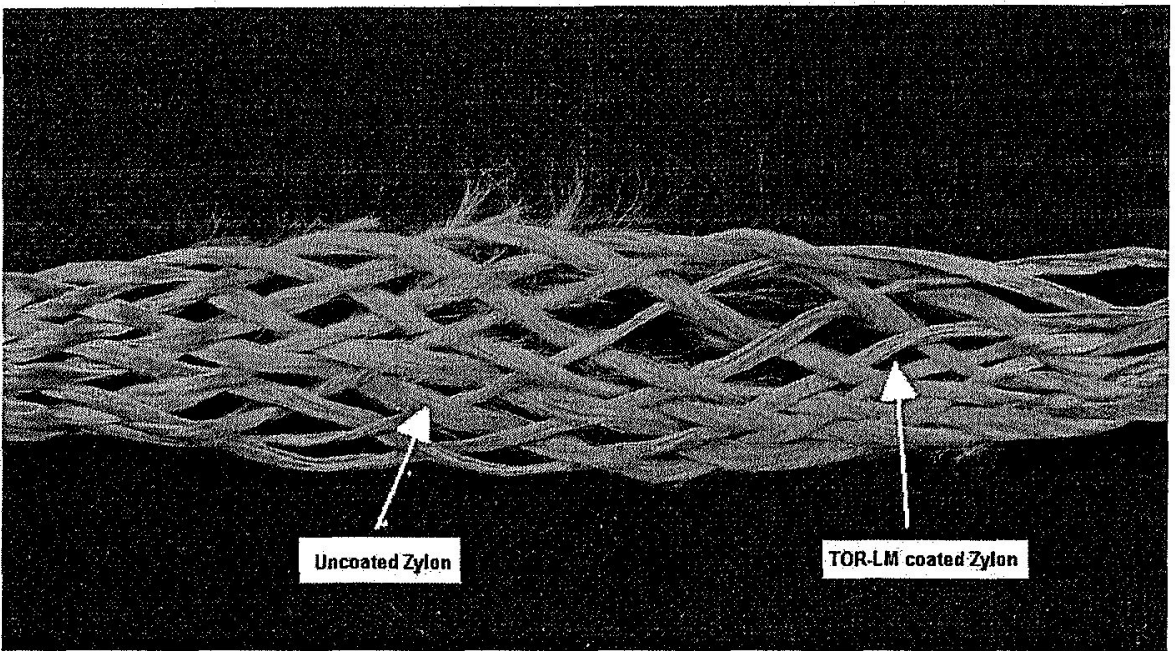

Figure 4: TOR-LM ${ }^{\mathrm{TM}}$ coated Zylon® Hoytether ${ }^{\mathrm{TM}}$, after exposure to AO the uncoated Zylon $\circledast$ (Figure 4). From a visual perspective, M5 with POSS were able to survive the testing regime without any apparent fraying or other damage. However, uncoated M5 cannot survive the harsh AO/UV environment, and it appears as though the POSS samples were not fully coated or the silicon is unable to fully protect the fibers in certain areas.

LMSS's tether samples included uncoated Zylon $\circledast$, Zylon® braid with Al PVD coating, Zylon® braid with $\mathrm{Al} / \mathrm{Al}_{2} \mathrm{O}_{3}$ PVD coating, Zylon® braid encapsulated within biocomponent Mylar/polyethylene facesheet with $\mathrm{Al}$ PVD coating, Zylon $\left(\right.$ braid encapsulated within biocomponent Mylar/polyethylene facesheet with $\mathrm{Al}_{1} \mathrm{Al}_{2} \mathrm{O}_{3} \mathrm{PVD}$ coating, and Zylon $\circledast$ braid with Triton Systems TOR-LM ${ }^{\mathrm{TM}}$ coating. These samples were exposed to approximately $5 \times 10^{20}$ atoms $/ \mathrm{cm}^{2}$ of atomic oxygen of $5 \mathrm{eV}$ energy. Samples were again characterized by photography and mass change. However, no tensile testing was performed on the LMSS samples due to unavailability of reasonably sized, coated samples. The visual observations include: the uncoated PBO braid with sizing showed very poor resistance to AO degradation, the PBO braid with aluminum PVD coating showed poor resistance to AO erosion (apparently the coating was not thick enough to adequately protect the PBO), and the PBO braid with aluminum and alumina PVD coating, had similar results to the previous sample. The encapsulated (facesheets) PBO braid with aluminum PVD coating was basically unchanged by AO exposure, and the encapsulated PBO braid with aluminum/alumina coating, was basically. unchanged. The PBO braid with TOR-LM ${ }^{\mathrm{TM}}$ coating was slightly bleached by $\mathrm{AO}$ whereas the fibers did not pull free as they did in other samples, and the PBO braid with TOR-NC ${ }^{\mathrm{TM}}$ coating, was very similar to the previous sample, though not as much bleaching. The mass change for these samples was not severe.

All coatings did improve the AO resistance of the PBO braid. However, only one coating showed the potential of full AO-resistance; nickel coated Zylon $\circledast$. However, it is predicted that the nickel coating would be too massive for any MXER type applications. It can be concluded that the aluminum/alumina coating and possibly the 
$\mathrm{TOR}^{\mathrm{TM}}$ coating showed signs of promise. However, the test results were somewhat sporadic due to the handling and unavailability of additional fresh samples by the end of the program.

Additional tensile testing on uncoated tether samples was performed at the Tethers Unlimited location in Bethell, Washington. Samples were braided into a Hoytether ${ }^{\mathrm{TM}}$ configuration and pull tested. The graph in Figure 5 illustrates the tension capability of a Zylon® Hoytether $^{\mathrm{TM}}$. The graph indicates that a Hoytether ${ }^{\mathrm{TM}}$ is capable of supporting a load in excess of the sum of the primary strand capabilities, indicating that when the tether is stressed beyond its "design" load, the secondary strands accept some load and do not degrade the strength of the primary lines when constructed in a Hoytether ${ }^{\mathrm{TM}}$ configuration.

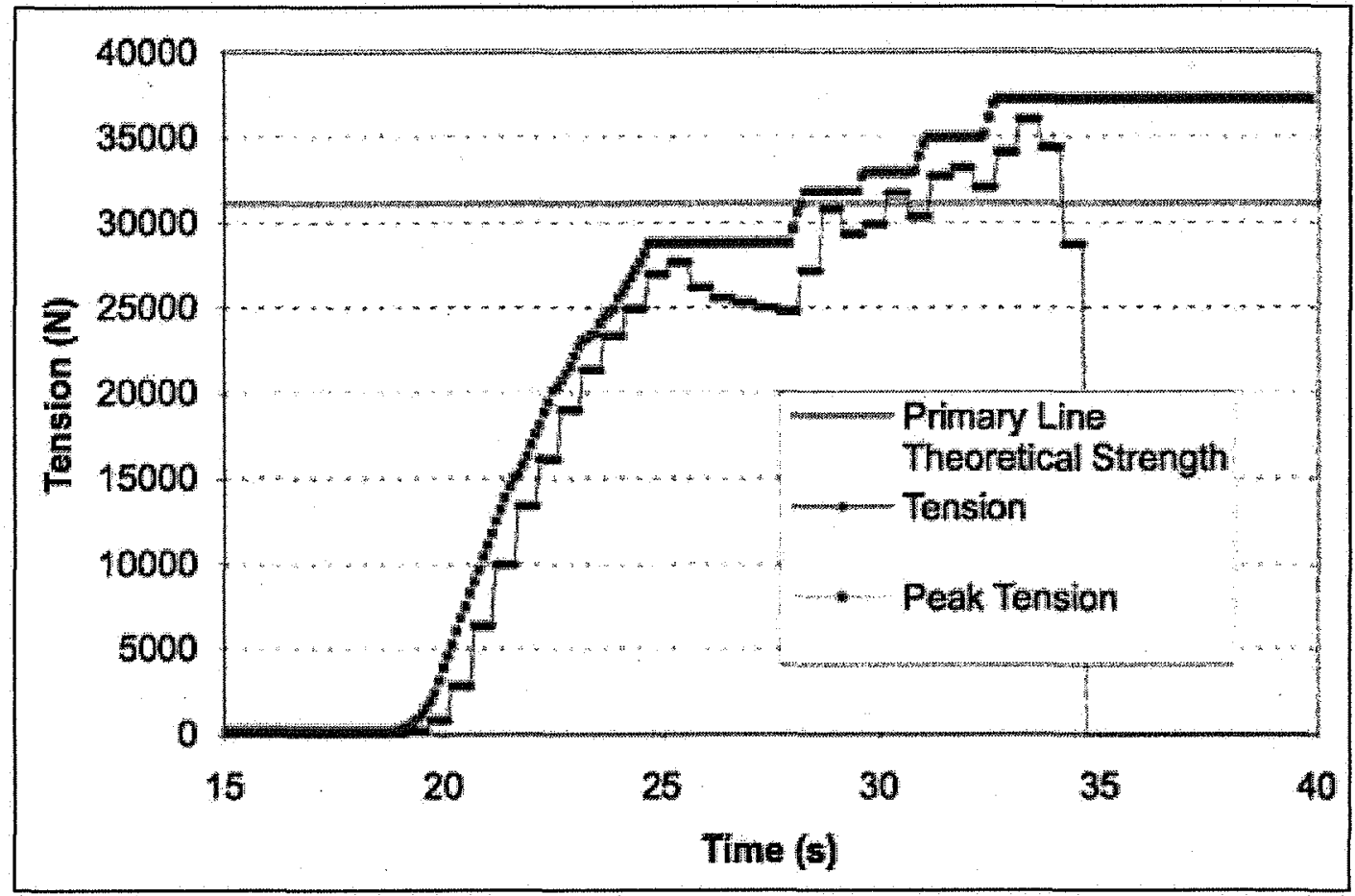

Figure 5: Tension Measurements on a Zylon ${ }^{\circledR}$ Hoytether ${ }^{\mathrm{TM}}$

\section{Propagator Code Development}

In order to execute a rendezvous between the tip of a rotating tether and a payload, the location of rendezvous must be known with extreme accuracy (on the order of meters) in inertial space prior to the actual rendezvous. This level of propagation accuracy would be challenging for any spacecraft; but is even more challenging for a hundredkilometer spinning dynamic structure like the MXER tether. It was recognized very early that typical orbital propagators would not be up to the challenge of such accuracy in a large dynamic structure. Therefore, a significant amount of research funding was directed towards attacking this problem, with no certainty that an attractive solution even existed.

Two teams, one from STAR and one from Tennessee Tech University, worked on the tether propagation problem. Each took a different, yet complementary, approach. STAR focused on developing a tether propagation algorithm that could achieve the desired accuracy yet run extremely fast on a computational platform. ${ }^{14}$ The principal theorist, Dr. Eugene Levin, was able to accomplish this by applying traditional, analytic mathematical techniques to the tether model that allowed orders-of-magnitude improvements in computational speed. Early on, he was able to achieve the desired accuracy in a model that included the full spectrum of idealized tether dynamic effects and external gravitation. Later, he addressed additional forces such as drag and solar radiation pressure, as well as realistic tether materials effects, such as material non-uniformity, creep, and mass loss due to erosion. He suggested that the simple addition of a laser ranging system between the deployer reel and the tether tip could allow the tether to adjust its length slightly and achieve the required accuracy, even under the combination of all these effects. 
The Tennessee Tech team, led principally by Dr. Stephen Canfield, pursued modeling techniques that were more computationally intensive, yet led to extremely accurate solutions. ${ }^{15}$ Such results could then be used to "benchmark" the accuracy of Dr. Levin's fast calculator. Canfield's team uncovered a number of dynamic effects that were not previously understood, such as certain long-period modes that could potentially interfere with rendezvous, that might not have been found with a less-rigorous model. In both cases, the remarkable results of these activities gave the program a dramatic increase in confidence that successful rendezvous could actually be achieved at realistic speeds and with straightforward operational techniques.

\section{Catch Mechanism Development}

The most advancement in any MXER subsystem has occurred in the catch mechanism development between LMSS and TTU. Before these contracts began, only a few conceptual approaches had been proposed (without analysis) for tether/payload rendezvous. One of the first tasks conducted in the capture mechanism development

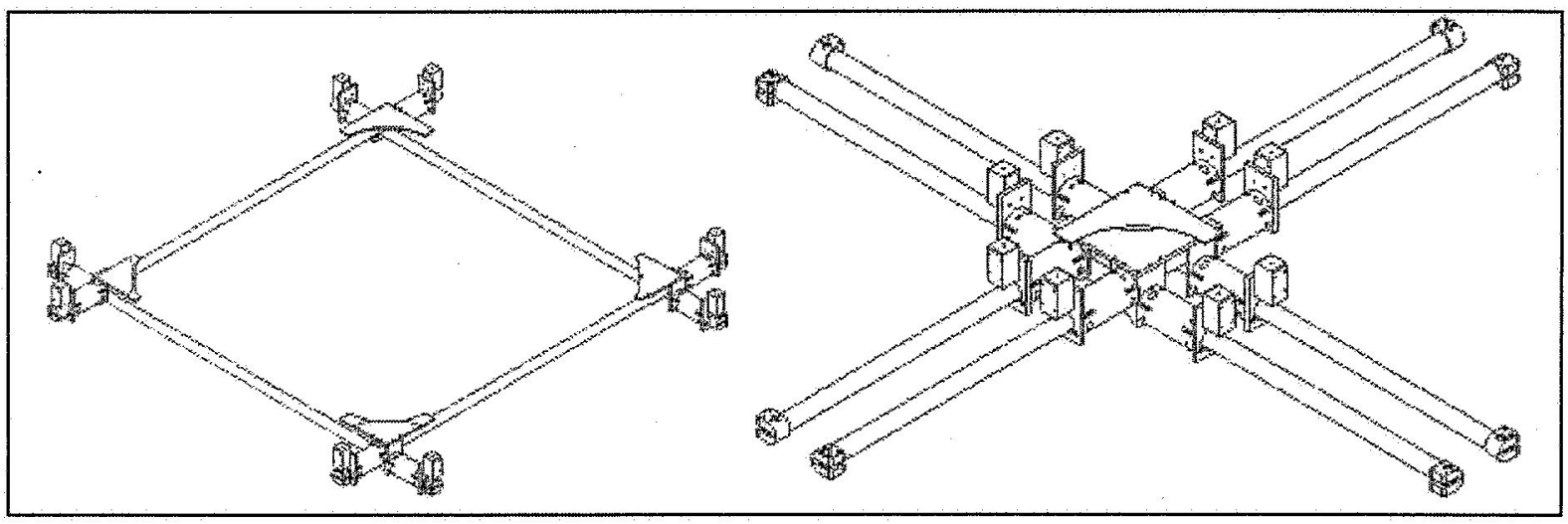

Figure 6: Schematic of QuadTrap in open (left) and closed (right) configurations

process was to create an estimate of the error window that would need to be accommodated by the capture mechanism. The error window was evaluated based on a monte-carlo simulation considering variation in a large number of tether and orbital parameters and initial conditions, and led to a prediction of the error window in the shape of an ellipse with defined major and minor axes. Between TTU and LMSS, nearly one-hundred concepts were generated and categorized according to fundamental operation principles in order to track and group proposed concepts based on their physical and kinematic methodology. Using the AHP (analytic hierarchy process) as a concept trade-study tool, the potential concept categories were reduced to a small number of concepts with higher potential for further examination. At the conclusion of the design process, AHP evaluation, and heuristic review, TTU chose and manufactured a single concept, calling it the "QuadTrap", while LMSS fabricated two concepts: the "umbrella" and the "PatTrap". It must also be noted that all catch mechanisms examined required different levels of modification to the payload. In the case of the QuadTrap and PatTrap, a slender, extendable, payload boom was required for the mechanism to close around while the umbrella concept had a boom with a hook to mate the boom with the catch mechanism.

The QuadTrap, shown schematically in Figure 6, consists of four slender beams, called spreader bars, and four corner nodes connected with eight prismatic joints, all arranged symmetrically. The capture mechanism is attached to the tether with four cables running from the tether tip to each corner node. As deployed, the catch mechanism is open (Figure 6 -left) until the payload boom penetrates the horizontal aperture of the quad-trap. At that time, the trap passively closes on the boom and seats against the boom's lever with the nodes guiding the payload to the center of the trap where all four corner nodes meet (Figure 6 - right). In this position, the QuadTrap has captured the payload. To release the payload, the payload's boom

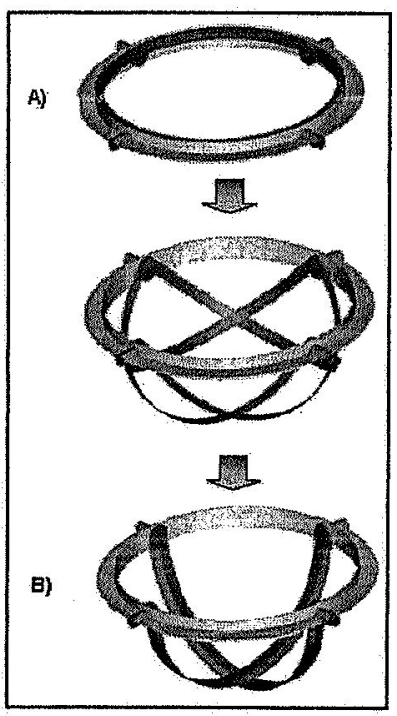

Figure 7: PatTrap 
simply reverses the mechanical lever which allows the boom to slide out of the catch mechanism. Three significant features of the QuadTrap are as follows: it can match the estimated error ellipse in an efficient fashion, it allows for passive actuation during closure, and its design passes all significant loads in tension through cable members.

The umbrella and the PatTrap were designed and manufactured at the LMSS facility in Denver, CO. The PatTrap is very similar to the QuadTrap in its operations. The PatTrap, shown in Figure 7, has a circular ring structure with four semi-circular bands that work in the same fashion as a traditional bear trap. Each set contains two bands that are connected at the bands endpoints to a single motor mount. The bands remain open (Figure 7A) until the payload boom penetrates the horizontal plane of the PatTrap. At this point, the two motors close each set around the payload boom which forces the payload to the center of the aperture and against the boom's lever. The capture is complete when each set's bands meet in the middle (Figure 7B) capturing the boom and payload in the center of the mechanism. To release the payload, the boom's mechanical lever will reverse and allow the boom to slide out of the catch mechanism. The umbrella appeared to be a derivative of the original baseline catch mechanism first mentioned in Sorensen's paper on MXER. ${ }^{16}$ The prototype was tested and the concept has three unique advantages: (1) no moving parts on the MXER side of the system, where repair would be extremely difficult, (2) no active sensing of payload/boom 'presence', and (3) the ability to accommodate the full 100 meter overtravel without colliding with the payload or having an impact load when the "slack" is taken up.

All three catch-mechanism engineering-models were evaluated in the TN Tech High Bay Facility in Cookeville, TN. The setup included a payload launch system using hydraulics to vertically launch a platform that would throw a "dummie" payload towards the catch mechanism. The payload used for the QuadTrap and PatTrap was configured as a cylindrical body with a stationary boom-like structure on the end. The PatTrap capture system was designed and tested at LMSS and shipped to TN Tech for further evaluation. Consequently, this design did not have the option for a test, evaluation, and alteration loop to optimize the design. As a result, the PatTrap system did not perform well due to high costs prohibiting the purchase of appropriately-sized motors to drive the circular rings. Consequently, the PatTrap's motor system did not travel fast enough to capture the "dummy" payload.

TN Tech employed an ADAMS simulation model to replicate the testing environment. Due to the developed ADAMS models and extensive testing at TN Tech, the QuadTrap performed on the order of several hundred simulated catches (Figure 8)! while capturing approximately $>90 \%$ of the time. TN Tech also incorporated accelerometers and automated the capture sequence with ranging devices. After the incorporation of the automated catch actuation, the QuadTrap laboratory hardware successfully captured the payload in $100 \%$ of the attempts! The experimental data collected with the accelerometers corresponded well with predicted data from a second

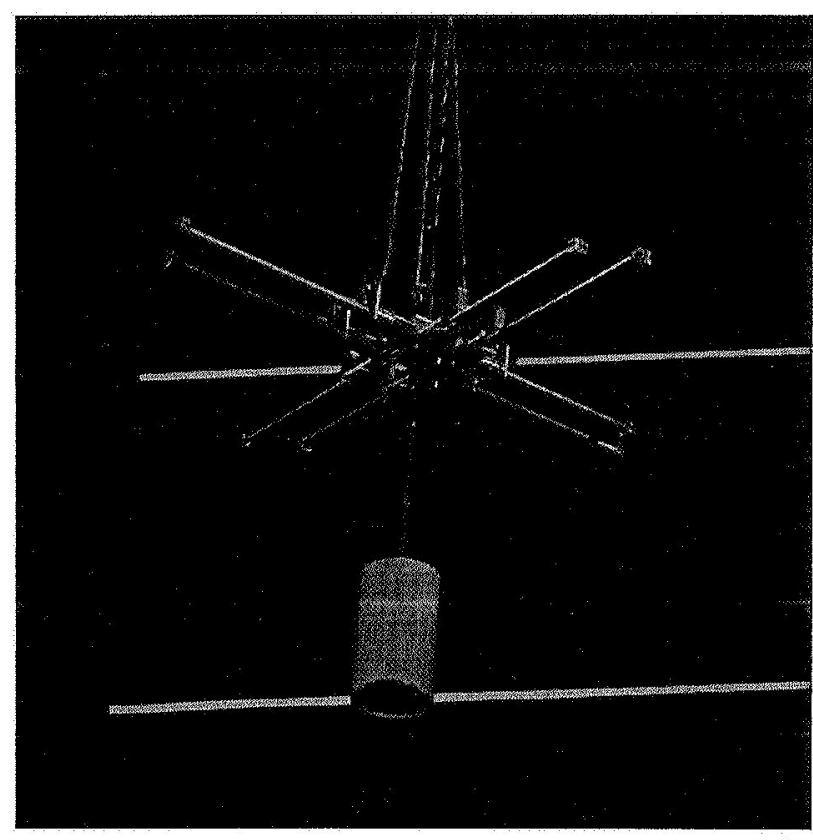

Figure 8: Successful Catch with QuadTrap ADAMS model developed for this capture scenario.

\section{E. Plasma Contactor Development}

The plasma contactor development applicable to MXER tethers has been mostly developed under two Phase II SBIR awards by SRS Technologies. One Phase II task focused on the feasibility and development of a Grid-sphere Anode for electrodynamic tethers, including MXER tethers. ${ }^{17}$ The grid-sphere concept includes a spherical, metallic grid that incorporates an outer layer of photolyzable material. Once deployed in space by gas pressure, the outer "balloon" material will photolyze, or melt away, leaving only the metallic grid structure remaining. The grid would then, with voltage applied, act as a spherical anode collecting electrons. SRS designed and built a 5-m prototypical grid-sphere. The plans included performing an inflation test and re-packaging this $5-\mathrm{m}$ prototype into a canister for future inflation test inside vacuum. However, during the manufacturing of the grid-sphere, the lights in the assembly area photolyzed the outer material enough that the material became embrittled with tiny holes. The 
inflation test worked well, but the grid-sphere was rolled into a container instead of the pre-planned folding process and stowed with further testing cancelled.

The other Phase II SBIR focused on the development of an innovative approach for contacting the plasma. ${ }^{19} 20$ The Solid Expellant Plasma Source/Contactor (SOLEX) is a new plasma generation technology based on physical processes discovered fortuitously during the Tether Satellite System Reflight mission (TSS-1R). The SOLEX will provide an efficient, robust and low-mass plasma generator for several applications including, spacecraft charge control, electrodynamic tethers and electric propulsion thrusters. Parallel computational work was conducted at NASA MSFC to lay a theoretical baseline for the classical bare wire collector and a tape configuration for future trade space analysis. ${ }^{21}$ During this time, an independent physics review was held to consider plasma collection options and implication on MXER. Their findings substantiated the SOLEX-like approach and practicality of MXER operations from a fundamental physics viewpoint.

\section{F. Conductive Tether/Coatings Development}

Two recent tasks have been devoted to the development of conductive tethers and coatings. Orbital Technologies completed its work under a Phase II STTR, which was centered on the development of technologies and analysis of another tethered flight experiment called the Tether Electrodynamic Spinup and Survivability Experiment (TESSX). ${ }^{22}{ }^{23}$ TESSX consists of two end-masses connected by a tether that was originally planned to create the spin by electrodynamic forces acting on the tether. However, trade studies indicated that modifying the spin rate through variable tether lengths is more appropriate. The system is designed for space spin-up in order to generate centripetal forces and create an artificial gravity environment. Prescribed gravity levels can be obtained to simulate Lunar ( $1 / 6 \mathrm{~g})$, Martian ( $3 / 8 \mathrm{~g})$, and other gravity environments for technology and hardware development (such as for in-situ utilization systems). This experiment would also allow for the study of biological effects for long-term exposure to zero and reduced gravity loads on astronauts.

An additional Phase II SBIR task was awarded to Triton Systems for the advancement of coatings onto polymer fibers. $^{24}{ }^{25}$ Triton Systems has been developing the process for the application and evaluation of lightweight metallic layers to the surface of high performance synthetic fibers to be used as conductive tethers. This task complemented the NRA tasks for the strength tether development whereas those coatings were developed to protect the tether against the harsh $\mathrm{AO} / \mathrm{VV}$ environment in space. This task focused on applying enough metallic material onto a non-metallic strength member in order for the tether to carry significant electrical current (see Figure 9). Products from this task would be applied to electrodynamic tethers as well as commercial electrical products.
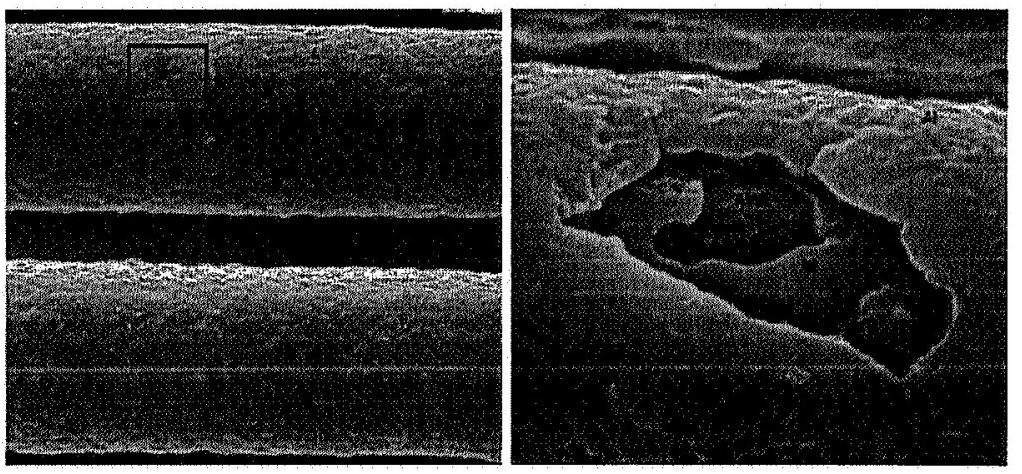

Figure 9: Scanning Electron Microscope pictures of Aluminum Plated onto Electroless Nickel Zylon® Fiber

\section{G. Deployer/Mechanisms Development}

Tethers Unlimited (TUI) developed various MXER technologies under a Phase II SBIR. The goal of this task was to research and analyze the overall MXER design while focusing on developing a deployer mechanism for a MXER-like tether. ${ }^{26}$ Another area of interest for this task included the analysis of a tether systems interaction with the catalogue of existing space debris/objects. ${ }^{27}$ TUI performed detailed conjunction analyses for a MXER-like tether $(\sim 100 \mathrm{~km}$ rotating MXER) and a MAST-like tether ( 1 km hanging tether) over periods of $12-24$ months. A major driver of operations cost for a MXER tether is dependent on this analysis and frequency determination, with preliminary results indicating little impact to such a tether system's operations.

Additional mechanism development has taken place under the Tennessee Tech Cycle II task which includes mechanism development for MXER and specifically the catch mechanism. This particular mechanism development included the initial investigation into a spatial joint, gimballing mechanism, called the "Canfield Joint" after its developer, Dr. Steve Canfield, for the complementary component to the catch mechanism, the payload boom. ${ }^{28}$ This mechanism, conceptually, could incorporate this gimbal to point the boom into the catch aperture for capture. 


\section{Future Development}

There are several technology gaps that must be closed prior to the development of a complete operational MXER system. Flight hardware gaps include: power storage and generation, successfully coating a multi-strand tether to meet UV and AO requirements, end-of-life (EOL) tether strength demonstration, production of an insulated high current capacity conductive multi-strand tether, electric emitters with sufficient lifetimes, electron collection, and validation of ED thrust predictions.

The power requirements of a MXER system are unique in that they require a large amount of energy collection over a long duration of a highly elliptical orbit and then a sustained high-current discharge near perigee. ${ }^{29}$ Flywheels have been baselined for the MXER design, however, the necessary flywheel systems have not been flight qualified. A parallel NASA research effort was sponsored at the Glenn Research Center (GRC) which provided the initial software models and designs of a MXER power system. Their work was based upon previous GRC flywheel hardware built for the International Space Station (ISS). ${ }^{30}$ At the required spin rates, the material strengths do not require further development and are sufficient for ground demonstration. The next step of the MXER power generation system would be ground test program for the development of a high energy density flywheel system capable of MXER charging and discharging power characteristics. Because the flywheel technology can reduce the MXER power system mass by more than $90 \%$, it may be enabling to MXER and a necessary subsystem technology. ${ }^{31}$

As indicated previously, there have been small investments in testing high strength materials after exposure to UV radiation and atomic oxygen. One of the most significant shortfalls of state-of-the-art (SOA) MXER technologies is the UV and AO material resistance. With a mass prohibitive nickel coated Zylon® as the only proven $\mathrm{AO}$ resistive coating, manufacturability improvement must be made in high strength tether materials. The next step of material validation clearly is the potential of coated M5®. M5® fiber is a synthetic high performance material produced by Magellan Systems, unit of DuPont Advanced Fiber Systems. M5® has unique characteristics that make it ideal for future tether systems including: higher strength-to-weight than Kevlar or Spectra, less brittle than carbon fiber, and resistant to ultra-violet radiation. ${ }^{32}$ The polymer structure of M5® may also allow for improved bonding characteristics of the coating required to prevent atomic oxygen degradation. Validation must include successfully testing the tensile strength after a sufficient exposure to UV and AO.

One of the most difficult challenges appears to be the successful production of a multi-strand hybrid tether. The strength tether portion of the MXER system has met the challenges in braiding secondary fibers that can successfully transfer the tensile loads after micrometeoroid impacts sever primary lines. However, for the conducting fibers, a similar interconnection must be made to shunt current in a fashion that allows for post-braiding insulation coating. It is likely that the solution will be a fiber that must be coated prior to braiding and applying a new methodology to ensure positive electrical contact at the interwoven positions (a significant engineering challenge). This conductive system must be able to successfully transfer the current loads after micrometeoroid impact degradation. Also, the severed strands will no longer be isolated from the external plasma, having the potential to lead to shorting the ED circuit. However, potential solutions have been discussed to alleviate the concern of shorting the circuit. While a flight demonstration is preferred, a ground demonstration program may be sufficient to demonstrate such a system. Vacuum facilities can maintain an ionospheric relevant environment. The hybrid tether would need to demonstrate both the transfer of current loads and resistance to shorting within the plasma. Also, the tether creeping that is likely to occur within the hybrid portion of the tether must account for the electrical characteristics of the conductive wire that will vary as the material stretches.

New electron emitter technologies, such as field emission cathodes, are advancing at a rapid pace due to the need of numerous commercial applications to replace hollow cathodes. While MXER currents are well beyond common applications, the necessary development of erosion resistant, long life hardware may be overcome without further MXER specific investment. A ground demonstration will be required to verify high current generation for the required lifetimes, but this is not predicted to incur a high Research and Development Degree of Difficulty. ${ }^{33}$

While much of the MXER technologies can be advanced to a TRL of $6^{34}$, there are MXER technology unknowns that must be validated through flight experiments with in-situ diagnostics. A fundamental concern of electrodynamic tether technology is the amount of electrical current that can be collected from the ionosphere by a given anode. SOA plasma contactors would require anode masses that are prohibitive. The SRS Technologies gridsphere anode may provide superior current collection with lightweight inflatable anodes. A technology demonstrator experiment could consist of a conductive tether with a grid-sphere at one end and a cathode on the other. The tether system's velocity though the magnetic field would generate the voltage needed to positively bias the anode and begin current collection. By varying the bias voltage and measuring the current in the tether, the performance of the anode could be characterized. Nominally, the experiment would be conducted in a highly 
elliptical orbit to obtain performance measurements at various altitudes and ion/electron densities. The results could be used to validate current collection models and provide confidence in future performance predictions.

More aggressive ED and MXER tether systems can also benefit through current collection beyond the ionosphere. The SRS SOLEX concept has the potential to enhance current collection beyond the ionosphere by serving as a plasma source for the anode. ${ }^{35}$ The successful implementation of a SOLEX device will have a significant impact to the MXER re-boost duration and therefore system usage rate, by increasing the useful re-boost phase of the orbit to extend well beyond the ionosphere. Like the grid-sphere, the SOLEX current collection system will require flight demonstration to fully characterize the performance of the current collection over various altitudes and external plasma densities.

Lastly, electrodynamic force generation must also be validated through in-situ measurements during a flight experiment. While the mechanism of generating a force by passing a current through a magnetic field is clearly understood, the ED tether acts as one side of a circuit, with the available space plasma closing the circuit. Due to the voltages and plasma density, the circuit may form a plasma sheath along the tether or even short at a location between the anode and cathode when a sufficient potential has been reached, leading to inefficiencies. The formation of a double layer may also impact the performance of the ED tether. Double layers are free-standing potential structures that form the transition between two plasmas that are at different potentials.

The electron current and potential between secondary and primary strands of a multi-strand tether during degradation must also be characterized to fully validate the expected performance of a MXER system. While a flight demonstration of technologies will be necessary prior to the use of a MXER system, it is likely that a single, low-cost flight experiment can validate all of the required technologies for the MXER system.

\section{Conclusion}

The fundamental goal for the MXER tether system is the reduction of space transportation costs. ${ }^{36}$ Throughout this study, economic awareness was maintained to ensure technical or research interests did not obscure the financial "bottom-line". MXER must be a practical system in its design, development, deployment, operations and fulllifetime costs. No legitimate technical factor that would undermine the basic premise of this transportation concept was ever found, despite the huge number of potential problems that were promulgated from within and without the team. The single most amazing feature of the entire MXER preliminary design study was the consistent trend to better performance than initially anticipated. Even when there appeared an impassable technical issue, a solution would emerge that would not only keep the project alive, but also, it often provided some unexpected performance gain somewhere else. Much work still needs to be done before an operational system can be established. However, the next flight experiments and ground hardware developments are well defined, with the expertise and infrastructure now available to accomplish them. It now appears the experimental risk has been retired and enough technical data confirmed to proceed with conventional spacecraft engineering development.

The final impact of this MXER tether research effort will not be understood for many years. Not only in the advancement of space tethers, but also in the multitude of spin-off and related technologies that were invented. The metallic coatings research on high-strength polymers is a critical step for space survivable tethers, as well as advanced aircraft wiring, next generation electromagnets and special military applications. Commercial infrastructure is now available to produce complex, multi-strand cable configurations for space and defense applications. The propagator algorithm and MXER design code has demonstrated that high-power, user-friendly software can be developed in a relatively short time and without huge monetary investments.

Solving the problems of a very challenging system such as MXER has lead to breakthrough innovations. The primary innovation is the potential application of the "Canfield Joint" to almost every satellite power, communications and instrument tracking problem. Next is the boom rendezvous approach, which is an extraordinary answer to the exasperating automated space-docking dilemma. MXER power systems reduced the mass and complexity of the best batteries and power conditioning subsystems into a single flywheel storage device through dual-windings.

The individual SBIR/STTR programs that collectively supported the MXER development effort also had amazing derivatives. The MAST flight experiment will provide critical scientific measurements of the time-history of space debris particles in Low Earth Orbit (LEO). Not only is this presently of unknown science value, but also, it will significantly impact the existing space debris models, now suspected of being in error. The grid-sphere offers a simple, passive electron collection device in a small lightweight package, capable of tens of amps of current, while the SOLEX technology gives a vastly superior replacement to existing hollow cathodes flight hardware.

Finally, the least recognized accomplishment was the team effort put forth by all the participants. The work could not have gone as far and fast as it did using the conventional organizational attitude. The combination of big 
and small companies, NASA personnel, support contractors and university members all collectively sharing data and resources to advance a single technology is not commonly seen in the aerospace community today. This is unfortunate, as this development program clearly demonstrates the gigantic strides that can be made when so organized. A MXER system is a highly complex system of systems; one that if successfully developed would allow a host of space and terrestrial tether applications to be readily achieved. The program has gone beyond its goals and objectives, beyond what the funding could possibly obtain, and stretched into an unprecedented number of other activities.

\section{Acknowledgments}

The work described in this paper was funded in whole or in part by the In-Space Propulsion Technology Program, which is managed by NASA's Science Mission Directorate in Washington, D.C., and implemented by the In-Space Propulsion Technology Project at Marshall Space Flight Center in Huntsville, AL. The program objective is to develop in-space propulsion technologies that can enable or benefit near and mid-term NASA space science missions by significantly reducing cost, mass or travel times.

The authors wish to acknowledge important contributions by the MXER contractors, which include Bruce McCandless of Lockheed Martin Space Systems, Dan Gramer of Orbitec, Nobie Stone of SRS Technologies, Jerome Pearson \& Eugene Levin of Star Technology and Research, Steve Canfield of Tennessee Technological University, Robert Hoyt \& Nester Voronka of Tethers Unlimited, and Kent Blizard of Triton Systems. MSFC inhouse code development was supported by Tara Polsgrove, Dan Thomas and George Khazanov. 


\section{References}

${ }^{1}$ Farris, B., Eberle, B., Woodcock, G., and Negast, B., "Integrated In-Space Transportation Plan," NASA/CR-2002$212050,2002$.

${ }^{2}$ Bonometti, J. and Frame, K., "Space Tethers Programmatic Infusion Opportunities," $53^{\text {rd }}$ JANNAF Propulsion Meeting, Monterey, CA Dec. 5-8, 2005.

${ }^{3}$ Cosmo, M. L. and E. C. Lorenzini, "Tethers in Space Handbook," Smithsonian Astrophysical Observatory, MSFC Grant NAG8-1 160, 3rd Edition, December 1997.

${ }^{4}$ Hoyt, R.P. and Uphoff, C.W., "Cislunar Tether Transport System," Journal of Spacecraft and Rockets, Vol. 37, No. 2, March-April 2000, pp. 177-186.

${ }^{5}$ Hoyt, R., "Design and Simulation of a Tether Boost Facility for LEO $\Rightarrow$ GTO," AIAA $36^{\text {th }}$ Joint Propulsion Conference and Exhibit, Huntsville, AL, AIAA 2000-3866.

${ }^{6}$ Hoyt, R., Voronka, N., Slostad, J., Frank, S., Newton, T., Barnes, I., "Development of Space-Survivable HighTenacity Tethers," 53 ${ }^{\text {rd }}$ JANNAF Propulsion Meeting, Monterey, CA Dec. 5-8, 2005.

${ }^{7}$ Hoyt, R. and Voronka, N., "Technologies for MXER Tether Transportation Systems," Center for AeroSpace Information Technical Report Server - Final Report to NASA Contract NNMO4AA40C, Tethers Unlimited, Bothel, WA, 2006 (unpublished).

${ }^{8}$ McCandless, B. and Kustas, F., "Momentum Exchange Electrodynamic Reboost (MXER) Tether Technology Study: Final Comprehensive Report," Center for AeroSpace Information Technical Report Server - Final Report to NASA Contract NNM04AA99C, Lockheed Martin Space Systems, Denver, CO, 2006 (unpublished).

${ }^{9}$ McCandless, B., Kustas, F., Marshall, L., Lytle, B., and Hansen, P., "Design Concept for a Reusable/Propellantless MXER Tether Space Transportation System," $53^{\text {rd }}$ JANNAF Propulsion Meeting, Monterey, CA Dec. 5-8, 2005.

${ }^{10}$ Hoyt, R., Voronka, N., Slostad, J., and Newton, T., "The Multi-Application Survivable Tether (MAST) Experiment", Final Report to NASA Phase II STTR Contract NNM04AB21C, Tethers Unlimited, Inc., Bothell, WA, 2006 (unpublished).

${ }^{11}$ Hoyt, R., Slostad, J., Twiggs, R., "The Multi-Application Survivable Tether (MAST) Experiment," $39^{\text {th }}$ Joint Propulsion Conference, Huntsville, AL Jul 20-23, 2003.

${ }^{12}$ Gittemeier, K., Hawk, C., Finckenor, M., and Watts, E., "Space Environmental Effects on Coated Tether Materials," ALAA 41 ${ }^{\text {st }}$ Joint Propulsion Conference and Exhibit, Tucson, AZ, AIAA 2005-4433.

${ }^{13}$ Gittemeier, K. A., Hawk, C. W., Finckenor, M. M., and Watts, E., "Atomic Oxygen Effects on Coated Tether Materials," 43rd AIAA Aerospace Sciences Meeting, Jan 2005, Reno, NV. AIAA-2005-0660.

${ }^{14}$ Levin, E. M., Pearson, J., and Oldson, J. C., "Dynamics Simulation Model for Space Tethers," Center for AeroSpace Information Technical Report Server - Final Report to NASA Contract NNM04AB51C, Star Technology and Research, Inc., Mount Pleasant, SC, 2006 (unpublished).

${ }^{15}$ Canfield, S., "Developing Capture Mechanisms and High-Fidelity Dynamic Models for the MXER Tether System," Center for AeroSpace Information Technical Report Server - Final Report to NASA Contract

NNM04AB13C, Tennessee Technological University, Cookeville, TN, 2006 (unpublished).

${ }^{16}$ Sorensen, K., "Conceptual Design and Analysis of an MXER Tether Boost Station," AIAA Paper 2001-3915.

${ }^{17}$ Stone, N. H. and Moore, J. D., "Grid Sphere Electrodes used for Current Collection at the Positive Pole of Electrodynamic Tethers," 45th AIAA/ASME/ASCE/AHS/ASC Structures, Structural Dynamics and Materials Conference, AIAA 2004-1505.

${ }^{18}$ Stone, N. H., "Concept for Tether Current Collection Using Grid-Sphere Electrodes," Final Report to NASA Phase II SBIR Contract NNM04AA11C, SRS Technologies, Inc., Huntsville, AL, 2006 (unpublished).

${ }^{19}$ Stone, N. H., "A Solid Expellant Plasma Source/Contactor for Electrodynamic Tethers (SOLEX-2)," Final Report to NASA Phase II SBIR Contract NAS8-02105, SRS Technologies, Inc., Huntsville, AL, 2005 (unpublished).

${ }^{20}$ Stone, N. H., "Development of a Solid-Expellant Plasma Contactor (SOLEX): Harnessing the TSS-1R TetherBreak Physics," 53 JAN JANAF Propulsion Meeting, Monterey, CA Dec. 5-8, 2005.

${ }^{21}$ Khazanov, G. V., Krivorutsky, E.N., and Sorensen, K., "Analyses of Bare-Tether Systems as a Thruster for MXER Studies," 41st Joint Propulsion Conference and Exhibit, Tucson, AZ, AIAA 2005-4116.

${ }^{22}$ Gramer, D., Munson, S., Hoffman, J., Mazzoleni, A., Cosmo, M., Lorenzini, E., Davis, J., Johnson, J., "Final Report on TAGS II: Tether Electrodynamic Spinup and Survivability Experiment (TESSX)," OTC-GS-151-FR-061, NASA Contract NNM04AB20C, Orbital Technologies Corporation, (ORBITEC ${ }^{\mathrm{TM}}$ ), Madison, WI, 25 May 2006. 
${ }^{23}$ Cosmo, M., Lorenzini, E., Gramer, D., Hoffman, J., and Mazzoleni, A., "TESSX: A Mission for Space

Exploration with Tethers," $41^{\text {st }}$ Joint Propulsion Conference, Tucson, AZ Jul 10-13, 2005.

${ }^{24}$ Gavrin, A., Blizard, K., and Gonyer, R., "Materials for the Advancement of MXER Tether Design," Final Report to NASA Phase II SBIR Contract NNM05AA13C, Triton Systems, Inc., Chelmsford, MA, 2005 (unpublished).

${ }^{25}$ Gavrin, A., Blizard, K., and Gonyer, R., "Materials for the Advancement of MXER Tether Design," $53^{\text {rd }}$ JANNAF Propulsion Meeting, Monterey, CA Dec. 5-8, 2005.

${ }^{26}$ Hoyt, R., "Technologies for MXER tether transport systems," Final Report to NASA Phase II SBIR Contract NNM04AA10C, Tethers Unlimited, Inc., Bothell, WA, 2006 (unpublished).

${ }^{27}$ Hoyt, R. and Buller, J., "Analysis of the Interaction of Space Tethers with Catalogued Space Objects," $4 I^{s t}$ Joint Propulsion Conference and Exhibit, Tucson, AZ, AIAA 2005-4430.

${ }^{28}$ Canfield, S. L. and Reinholtz, C. F., "Development of an All-Revolute-Jointed Constant-Velocity Coupling," Journal of Applied Mechanisms \& Robotics, Vol. 2, No. 3, July 1995, pp. 13-19.

${ }^{29}$ Sorensen, K., "Momentum eXchange Electrodynamic Reboost (MXER) Tether Technology Assessment Group Final Report," NASA/MSFC In-Space Propulsion Technology Office, Huntsville, AL, 2003.

${ }^{30}$ Kascak, P. E., Kenny, B. H., Dever, T. P., Santiago, W., and Jansen, R. H., "International Space Station Bus Regulation with NASA Glenn Research Center Flywheel Energy Storage System Development Unit," NASA TM2001-211138, 2001.

${ }^{31}$ Kascak, P.E., Jansen, R. H., Dever, T., Sorensen, K., "Modeling and Optimization of a MXER Tether Power System," 53 $3^{\text {rd }}$ JANNAF Propulsion Meeting, Monterey, CA Dec. 5-8, 2005.

32 "Wikipedia: M5 fiber," Wikipedia: The Free Encyclopedia, [online database], URL: http://en.wikipedia.org/wiki/M5 fiber [cited 04 May 2006].

${ }^{33}$ Mankins, J. C., "Research \& Development Degree of Difficulty $\left(R \& D^{3}\right)$ : A White Paper," March 10, 1998, [online database], URL: http://www hq.nasa.gov/office/codeq/trl/r\&d3.pdf [cited 04 May 2006].

${ }^{34}$ Mankins, J. C., "Technology Readiness Levels: A White Paper," April 6, 1995, [online database], URL: http://www.hq.nasa.gov/office/codeq/trl/trl.pdf [cited 04 May 2006].

${ }^{35}$ Stone, N. H., "Conceptual Description: Electrodynamic Propulsion in a Vacuum-Field Planetary Environment," SRS Technologies, Inc., Huntsville, AL, 2005 (unpublished).

${ }^{36}$ Bonometti, J., Dankanich, J., and Frame, K., "Reusable Propulsion Architecture for Sustainable Low-Cost Access to Space," $53^{\text {rd }}$ JANNAF Propulsion Meeting, Monterey, CA Dec. 5-8, 2005. 


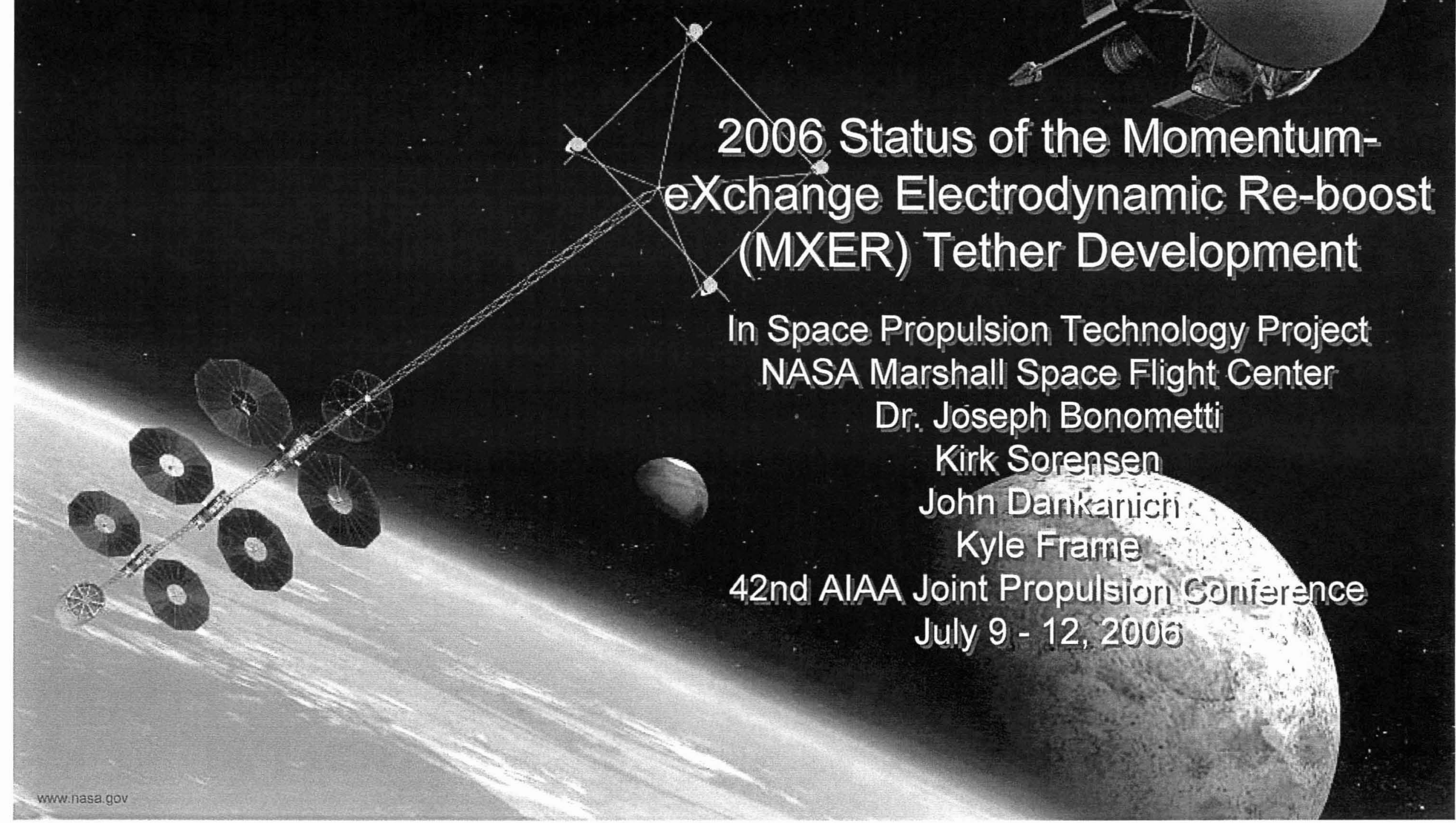




\section{Outline}

- Emerging Propulsion Technologies

- ISPT Entrance Process

- Program Status

- Momentum eXchange Electrodynamic Reboost (MXER) Tether Project

- Contract(s) Status

- Technical Accomplishments

- Future of the Technology

- On-going Research

- Suggestions for New Work

- Concluding Remarks 


\section{In-Space Propulsion Technology Project Prioritization Process}

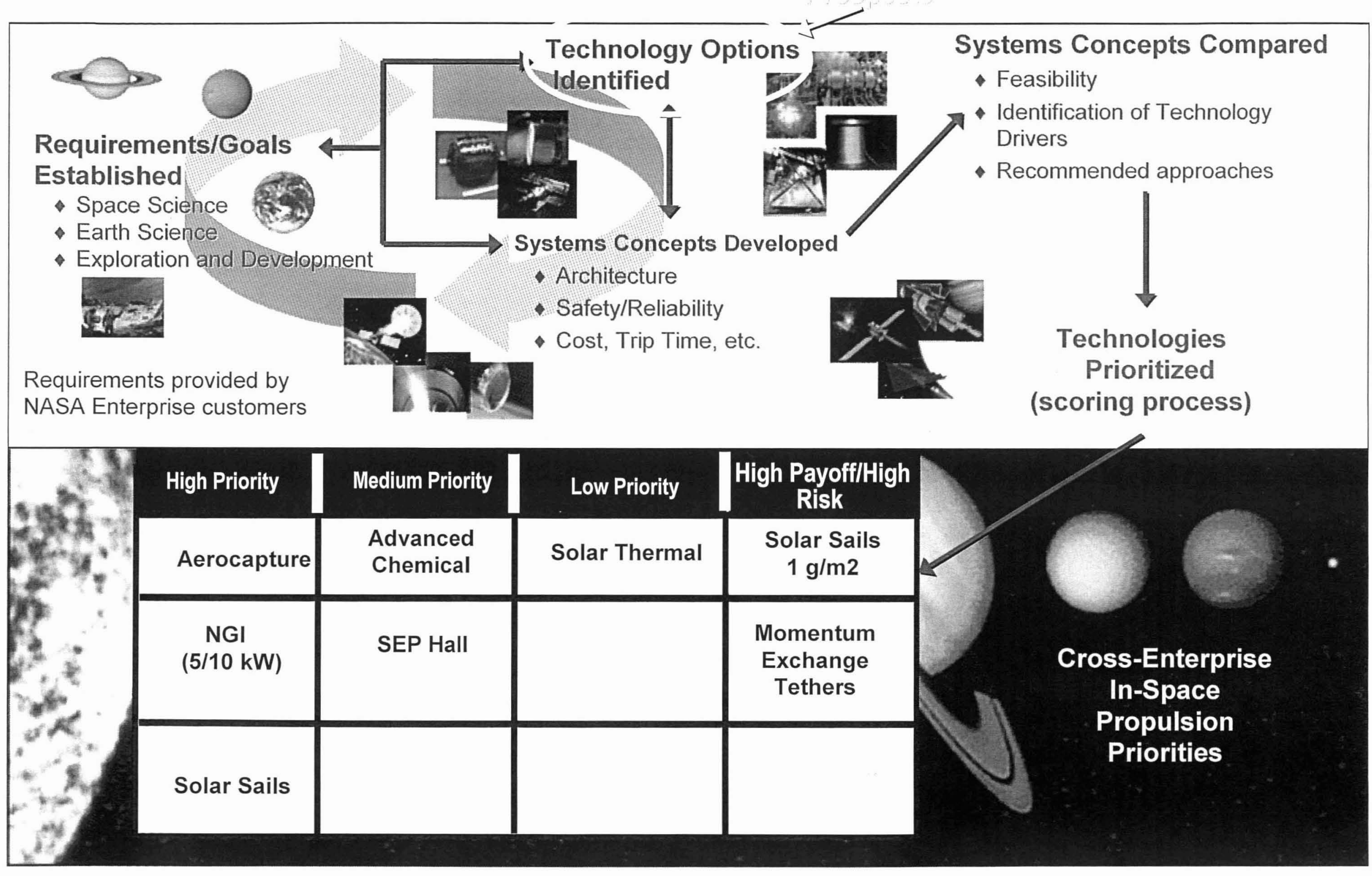


System Test, Launch \& Operations

System/Subsystem Development

Technology

Demonstration

Technology

Development

Research to Prove

Feasibility

Basic Technology Research

\begin{tabular}{|l|}
\hline TRL 9 \\
TRL 8 \\
- \\
TRL 7 \\
TRL 6 \\
TRL 5 \\
TRL 4 \\
TRL 3 \\
TRL 2 \\
TRL 1
\end{tabular}

NASA Implementation: (Deep Space One lon Engine Example)

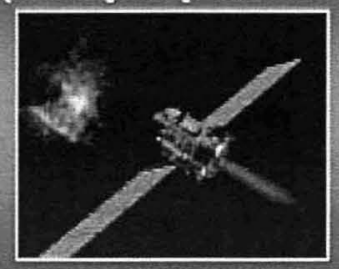

Aerocapture

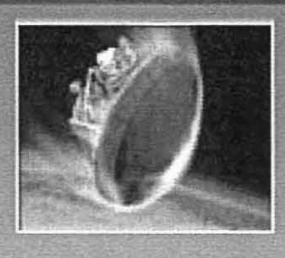

Solar Electric Propulsion
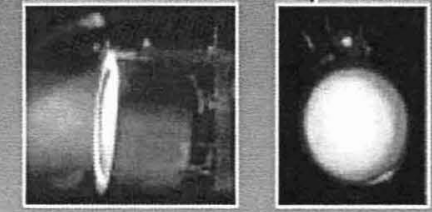

Solar Sails

In-Space Propulsion Technologies

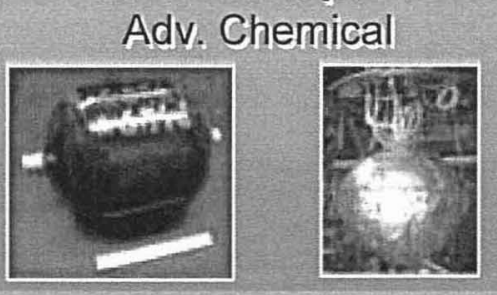

Tethers
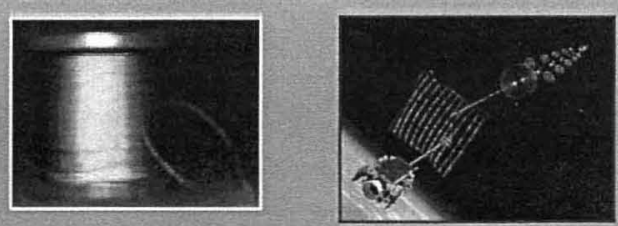

Low-TRL Technologies For the Future

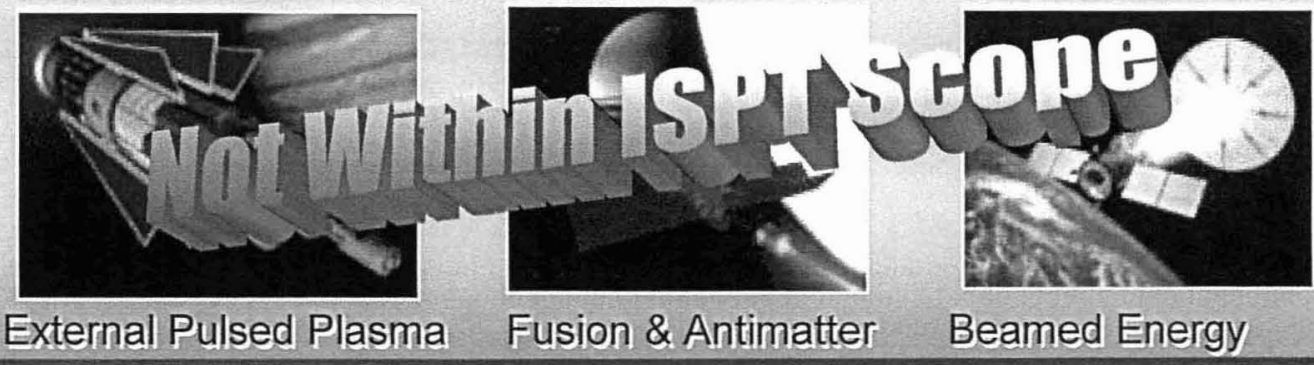




\section{Emerging Propulsion Technologies}

\section{Momentum-eXchange}

Electrodynamic-Reboost (MXER) Tethers

\section{Description}

A MXER tether is a long, multi-strand tether facility functioning as a reusable upper stage providing propellantless propulsion by utilizing momentum exchange and giving payloads a rapid increase in orbital energy and velocity. MXER can conceptually throw payloads from LEO to GTO and beyond.

\section{Potential Benefits}

- Little or no propellant required

- $>70 \%$ of Earth escape Delta-V provided

- Increases launch payload mass fraction

- Significantly lowers costs of commercial, Lunar, Mars, and other interplanetary missions.

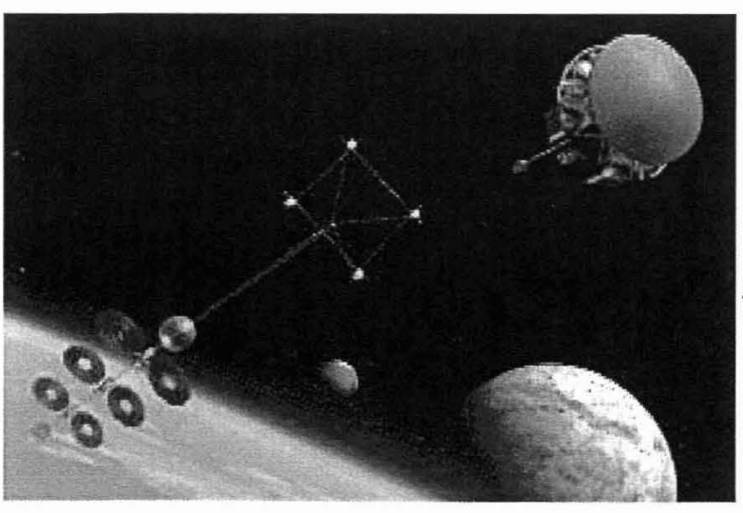

Artist's Concept of a MXER Tether
Emerging Propulsion Technologies

(EPT): Gateway into In-Space Propulsion Technology Project

\section{Description}

Newest area of In-Space that bridges technologies in the lower TRL range $(2 \& 3)$ to the mid TRL range (4 to 6 ). MXER tether has been the only successful top priority project with a rapidly advancing schedule. EPT is also home to secondary, candidate ISP technologies such as:

- Ultra-light solar sails

- Advanced electric concepts (FRC, PIT, etc.)

- ED or other tether applications

\section{Acquisition Process}

EPT has implemented a process for identifying, assessing, selecting/funding, reviewing, and infusing prospect technologies through the ISP Office. Prospect technologies are identified by:

- Request For Information (RFI)

- Conferences (NIAC, JPC, JANNAF, etc.)

- STTR/SBIR, unsolicited proposals
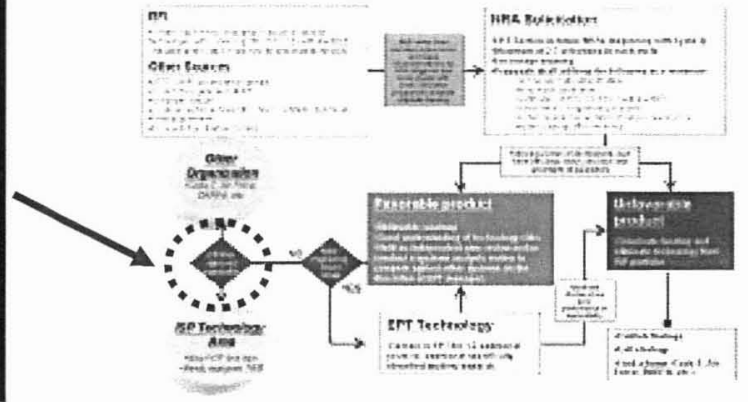

EPT Entrance Process
Related Technologies: Spin-off Technologies from sub-system investigations

Because of the unique mission of EPT, a novel technology development can lead to innovative solutions to non-propulsion problems. The investment made reaps further benefits for the science community as well as other users.

Examples of subsystem benefits from the MXER programs include:

> Canfield Joint - Instrument / solar panel / communications tracking without slip rings

> Science data on space micrometeoroid debris from the MAST experiment

$>$ SOLEX- A new plasma contactor with significant benefits over a traditional hollow cathode

$>$ High strength cables for space structures and terrestrial applications

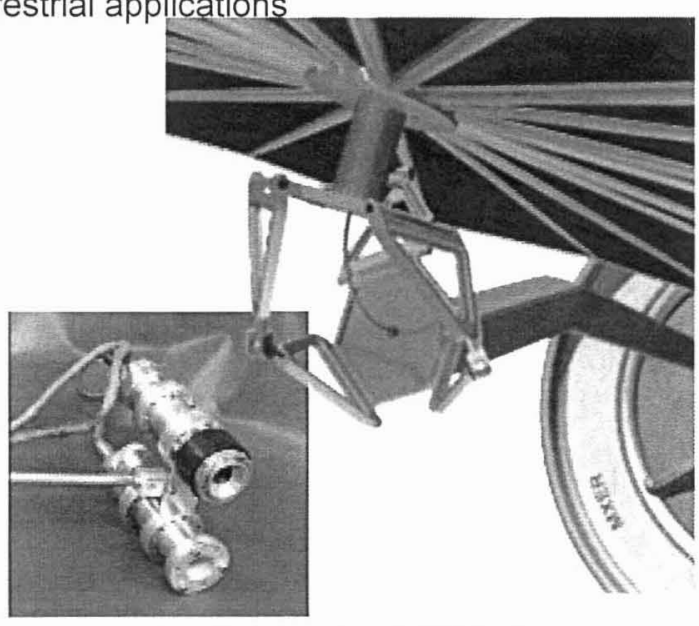

Canfield Joint \& SOLEX 


\section{MX and ED Tether Evolution}

\section{Masua contactoh}

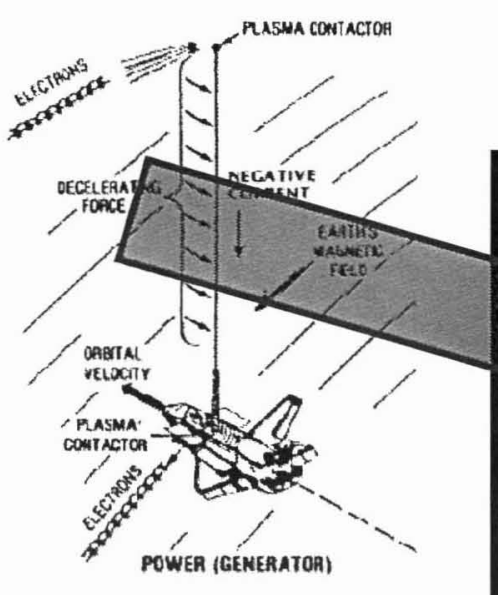

Colombo, Grossi (1976)

TSS-1 (1992)

TSS-1R (1996)

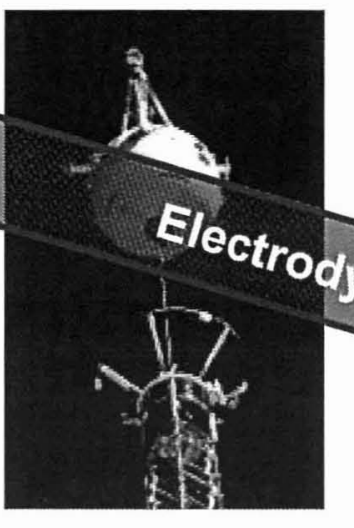

"Rotovator"

GEO Space Elevator

Artsutanov (1960) Isaacs, Vine (1966) Pearson (1975) Moravec (1978)
Artsutanov (1969)

Hypersonic Skyhook

$$
\text { ProSEDS (2003?) }
$$

PMG (1993)
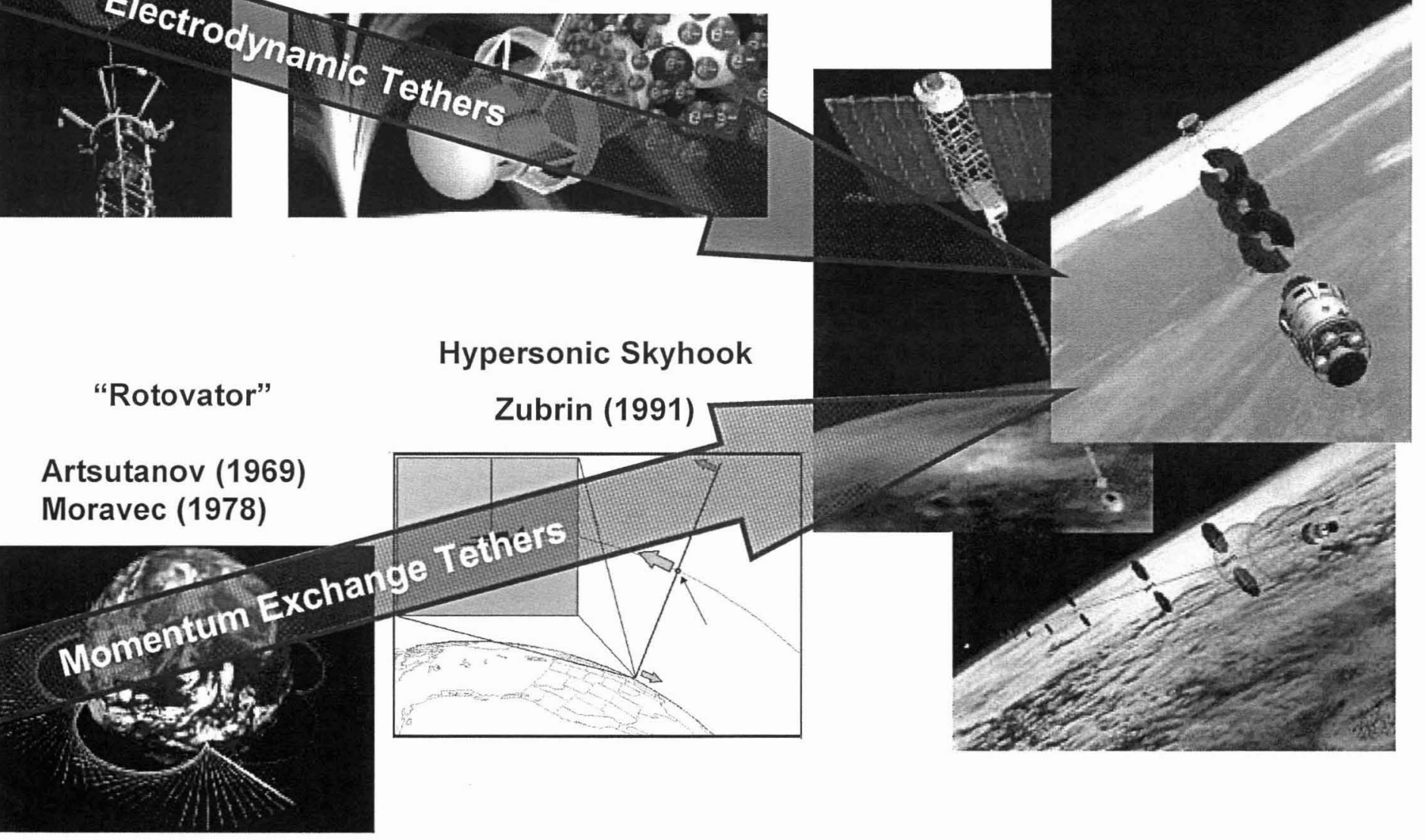

The MXER technology has had the time to mature, along with our understanding of the critical factors in implementation. 


\section{Momentum eXchange Electrodynamic Reboost (MXER) Tether}

- The MXER Tether is a reusable, propellantless, in-space "upper stage" for sending payloads from LEO to GTO and beyond.

- Long, rotating cable operating in an elliptical orbit.

- Restores orbital energy by using electrodynamic tether

- Development time: 10-15 years

- Potential benefits to payloads using MXER

- Increase in Isp while maintaining reasonable trip time

- Lowers overall mission cost and/or enables larger payloads

- Capable of 75\% Earth escape Delta-V

- Interplanetary mission enhancer

- Useable by essentially all missions beyond LEO

- A spiral development for future generations

- Tremendous future growth

- Readily scales up or down

- Future transportation to and from Lunar surface

- MXER generates launch vehicles savings

- Increase in payload size and capability

- Savings after only a few uses and significantly accumulate with more missions

- High equivalent Isp without long durations in radiation belts
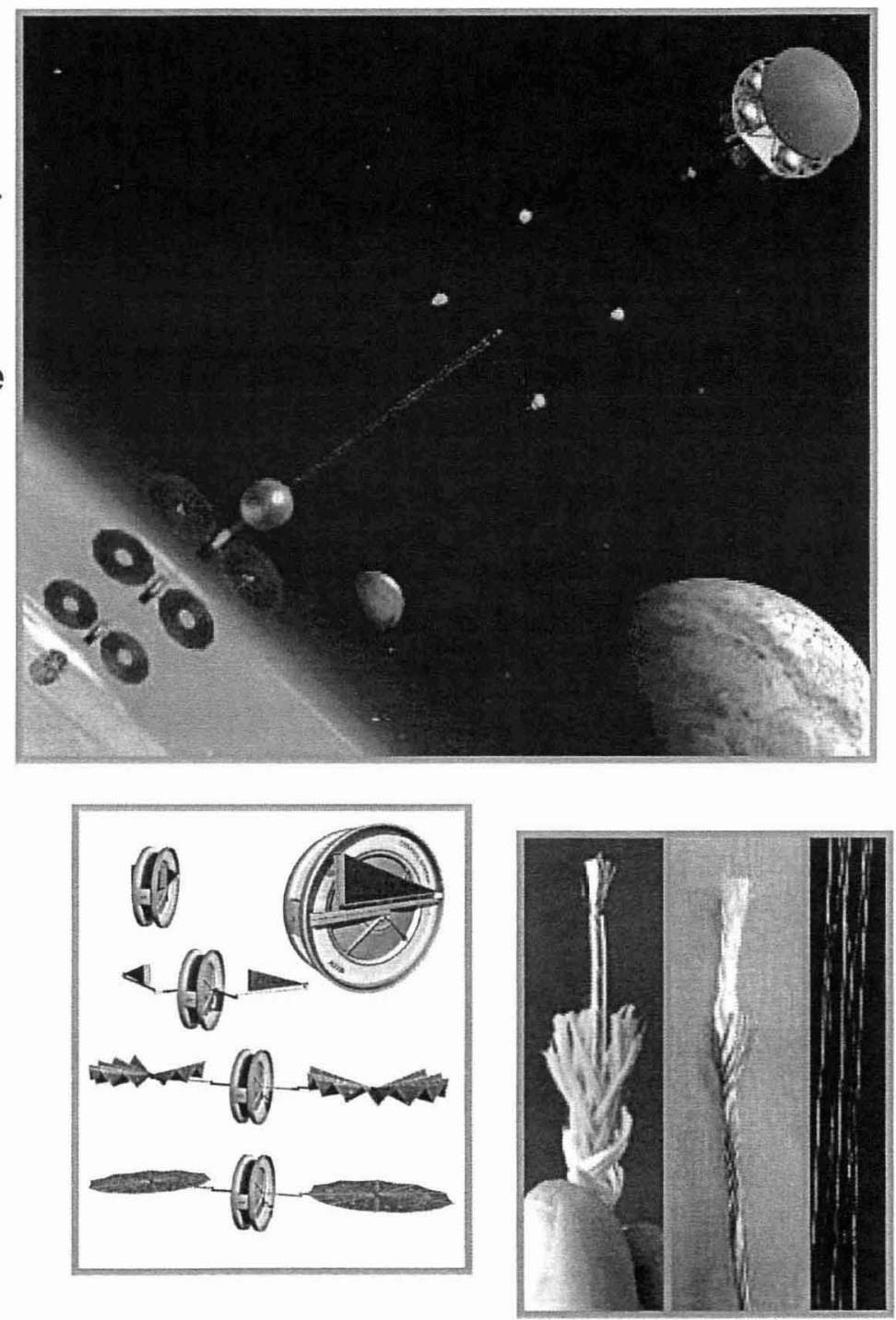


\section{Energy Needed for Interplanetary Missions}

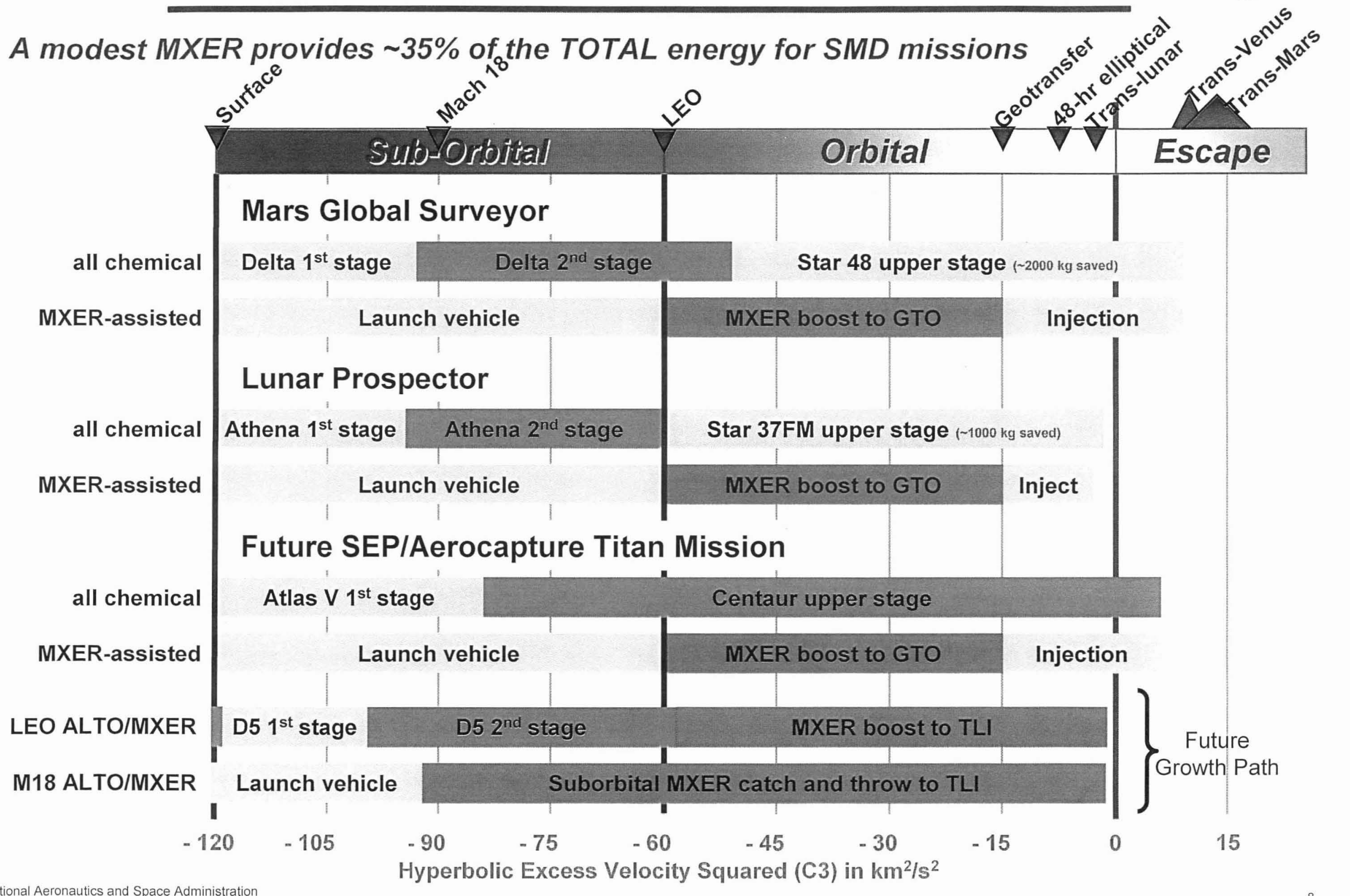




\section{Single Launch MXER Tether Concept}
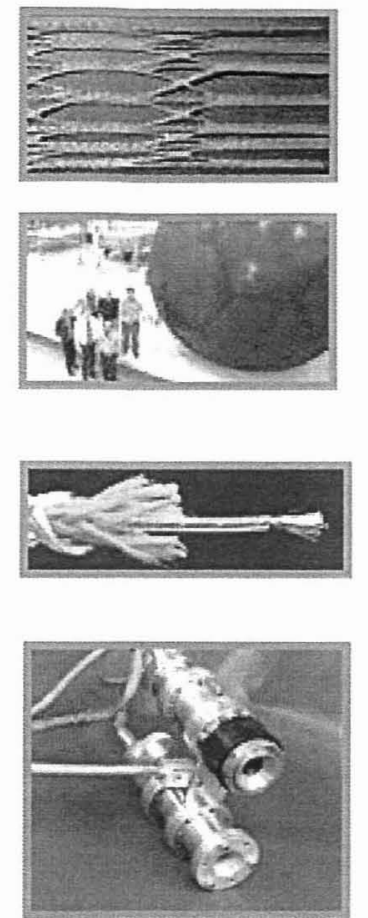

- Passive anode on each end collecting electrons

- Insulated aluminum fibers integrated into strength tether

- Active cathodes on each end emitting electrons

"QuadTrap" Catch Mechanism

\section{multi-strand}

configuration

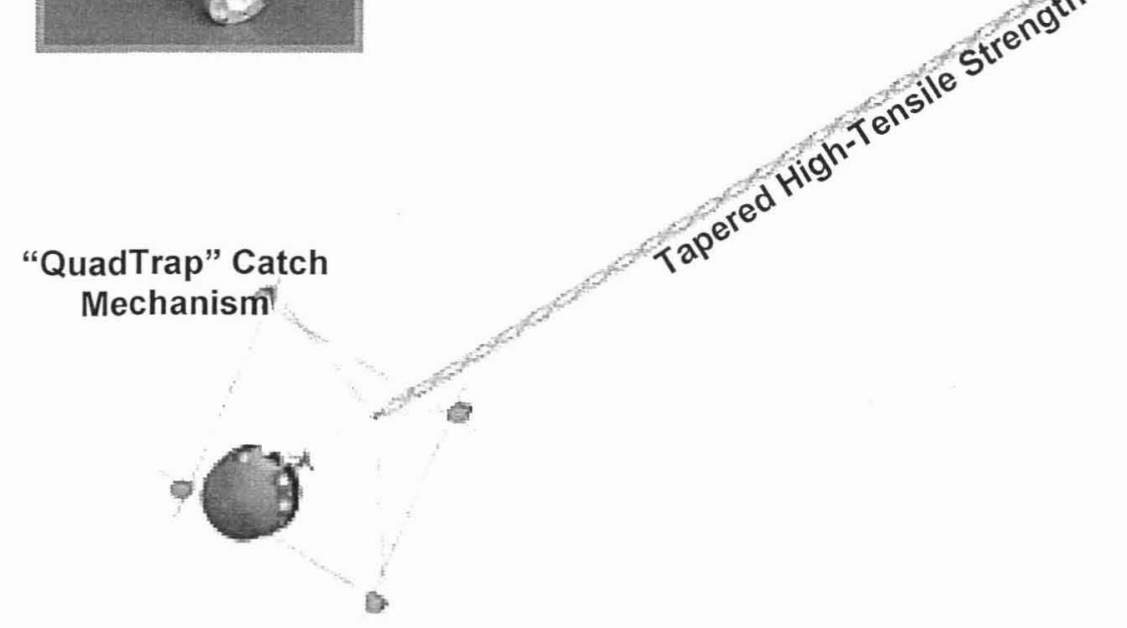

Power Nodes to store energy for electrodynamic

reboost

rrays to recharge flywheels

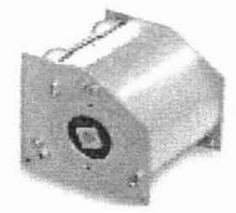

- Payload accommodation assembly

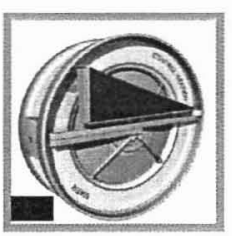

- Multiple control stations driving current through the tether

- Catch mechanism on one end capturing \& releasing prospective payloads 


\section{MXER Tether Propulsion}

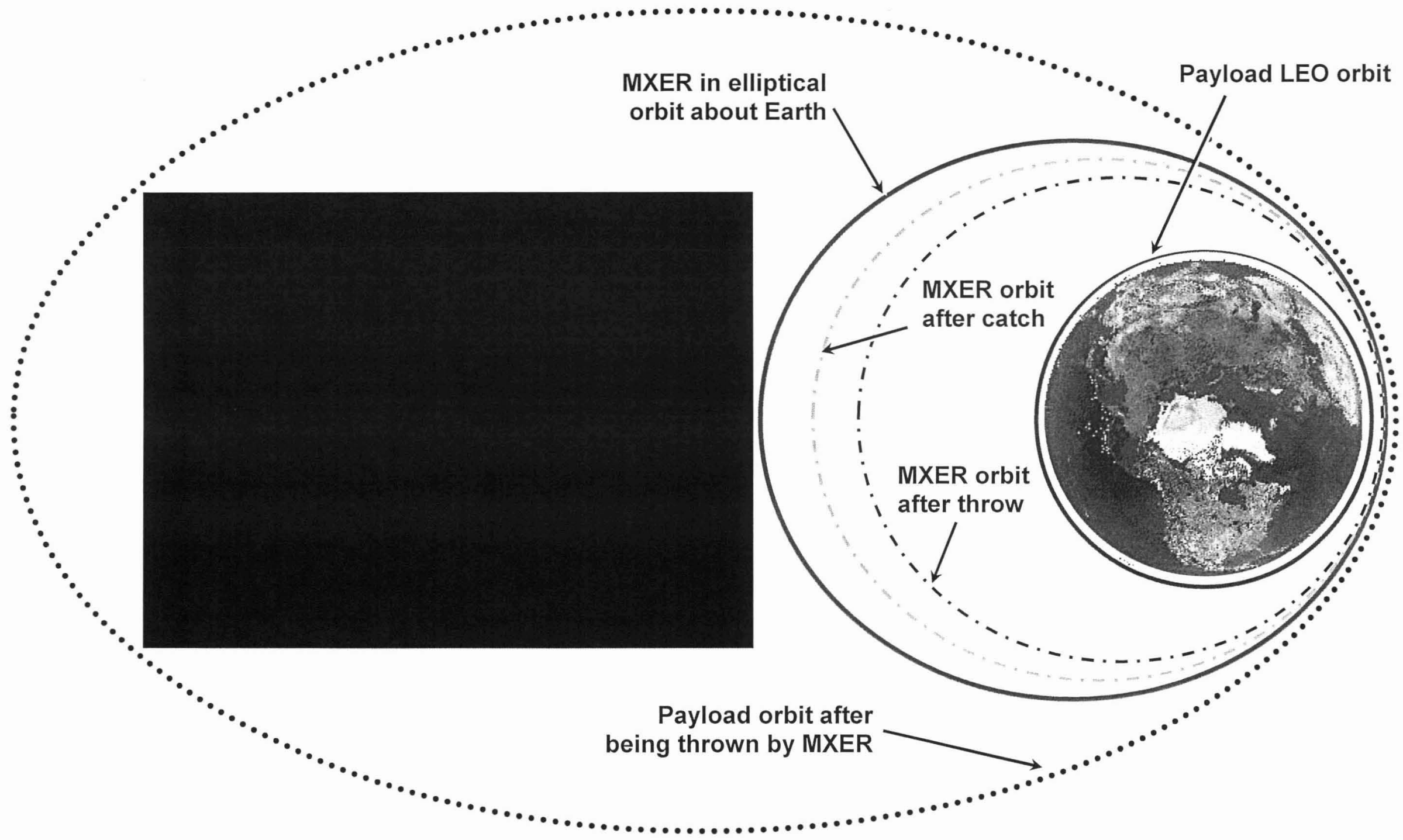


Electrodynamic Reboost

$\checkmark$ Over a period of 4-6 weeks, the tether reboosts from its post-throw orbit to its pre-catch orbit.

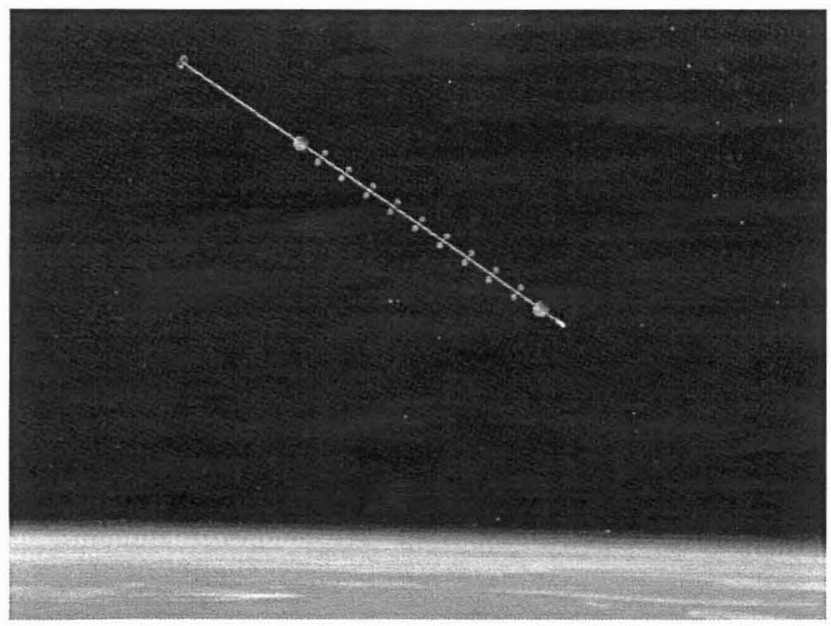

\section{s}

Illustration of electron

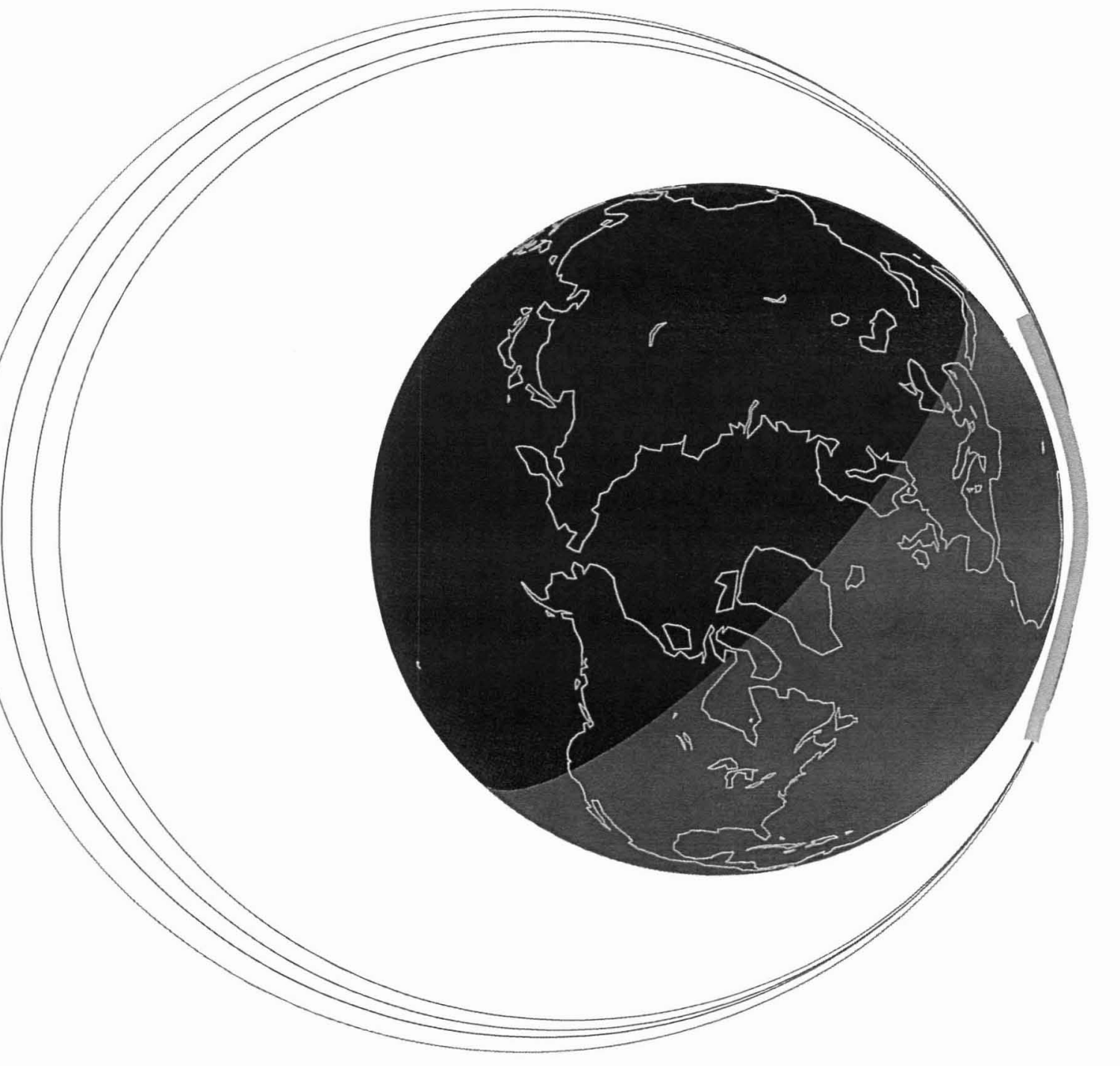
collection and emission 


\section{MXER Tether Strategic Technology Approach}

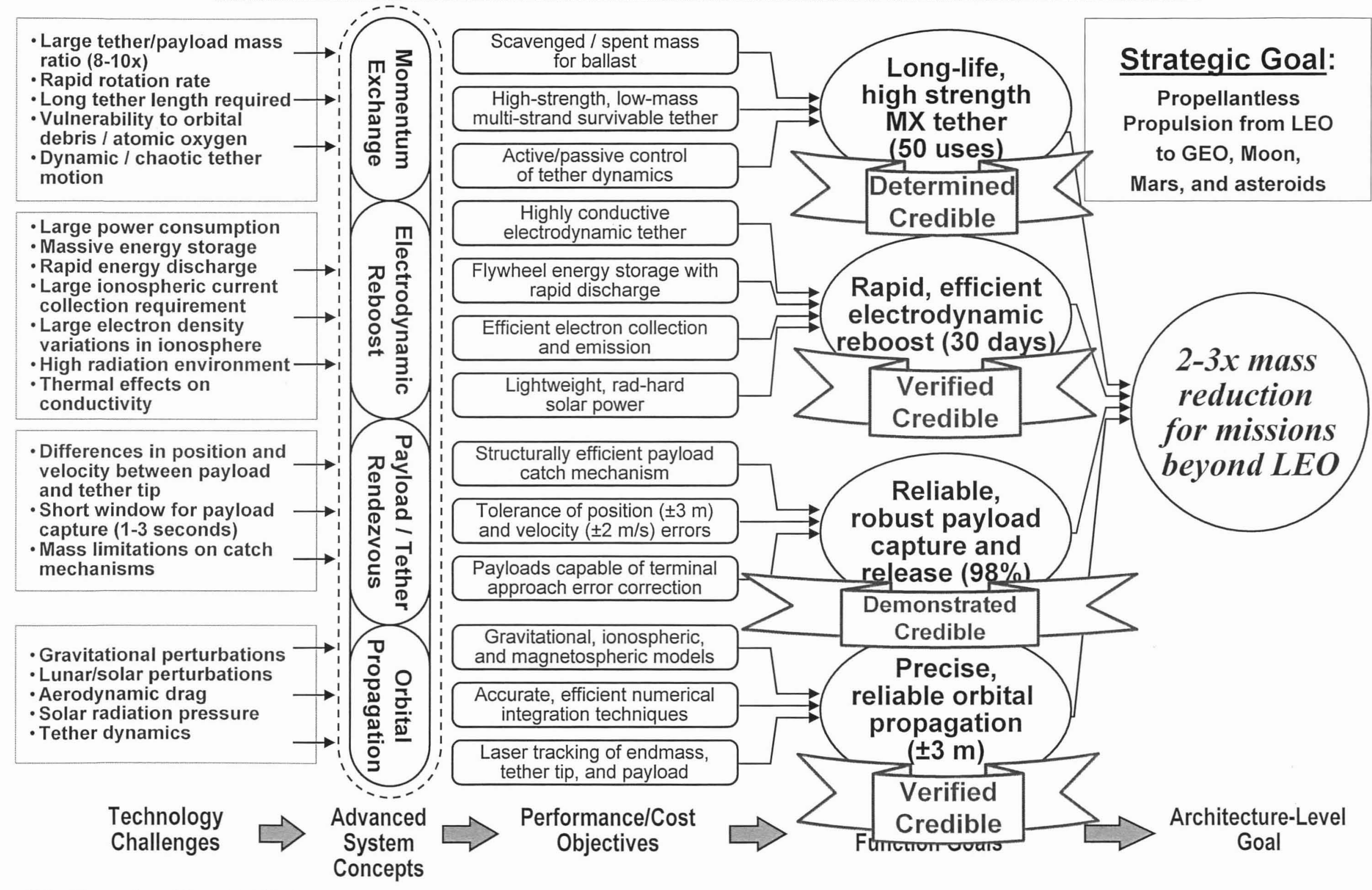




\section{MXER Tether Technology Tasks}

\section{Cycle 2 NRA contracts}

- Lockheed Martin Space Systems (LMSS)

- Catch Mechanism

- Strength tether

- Star Technology and Research, Inc. (STAR)

- Propagator/predictor code

- Tennessee Tech University (TTU)

- Catch Mechanism

- Propagator/predictor code

- Tethers Unlimited, Inc. (TUI)

- Propagator/predictor code

- Strength tether

\section{Directed Tasks}

- Marshall Space Flight Center (MSFC)

- Electrodynamic tether (FY03)

- Jet Propulsion Laboratory (JPL)

- Plasma contactor (FY04)

- Glenn Research Center (GRC)

- Power system (FY05 \& FY06)

- Phase II STTR contracts

- Orbital Technologies, Inc. (Orbitec)

- Spinning tether demo mission concept

- Tethers Unlimited, Inc.

- Strength tether demo mission concept
- Phase II SBIR contracts

- SRS Technologies, Inc. (SRS)

- Plasma contactor (anode)

- Plasma contactor (cathode)

- Tethers Unlimited, Inc.

- MXER technologies

- Triton Systems, Inc. (TSI)

- Electrodynamic tether

- Other Supporting Tasks

- GSRPS

- Georgia Tech

- University of Michigan

- Auburn Materials Research Contract

- MSFC in-house studies

- MSFC Material Testing

- Summer Facility Program

- U.S. Army Braiding Machine

- SBIR/STTR Phase I

- Industry R\&D 


\section{Strength Tether Development}

\section{LMSS and TUI}

- Tether must be coated or treated

- Atomic oxygen (AO) erosion

- Ultraviolet (UV) radiation embrittlement

- Consideration of coating's properties

- Absorptivity and emissivity

- Wear and handling characteristics

- Conductivity (both thermal and electrical)

- Mass impact

- Micrometeoroid and orbital debris (MM/OD) environment

- Multi-strand

- Cross-linked configuration

- Providing redundant load paths

\section{- Cycle II NRA Strength Tether Focus}

- Multi-strand tether cost-effective manufacturability

- Yarn plying/twisting

- Bobbin winding

- Braiding

- Spooling

- Coating and/or treatment process for protection from AO and UV

- Development of the hybrid portion of the tether (secondary goal)

- Contains both conductive and strength members

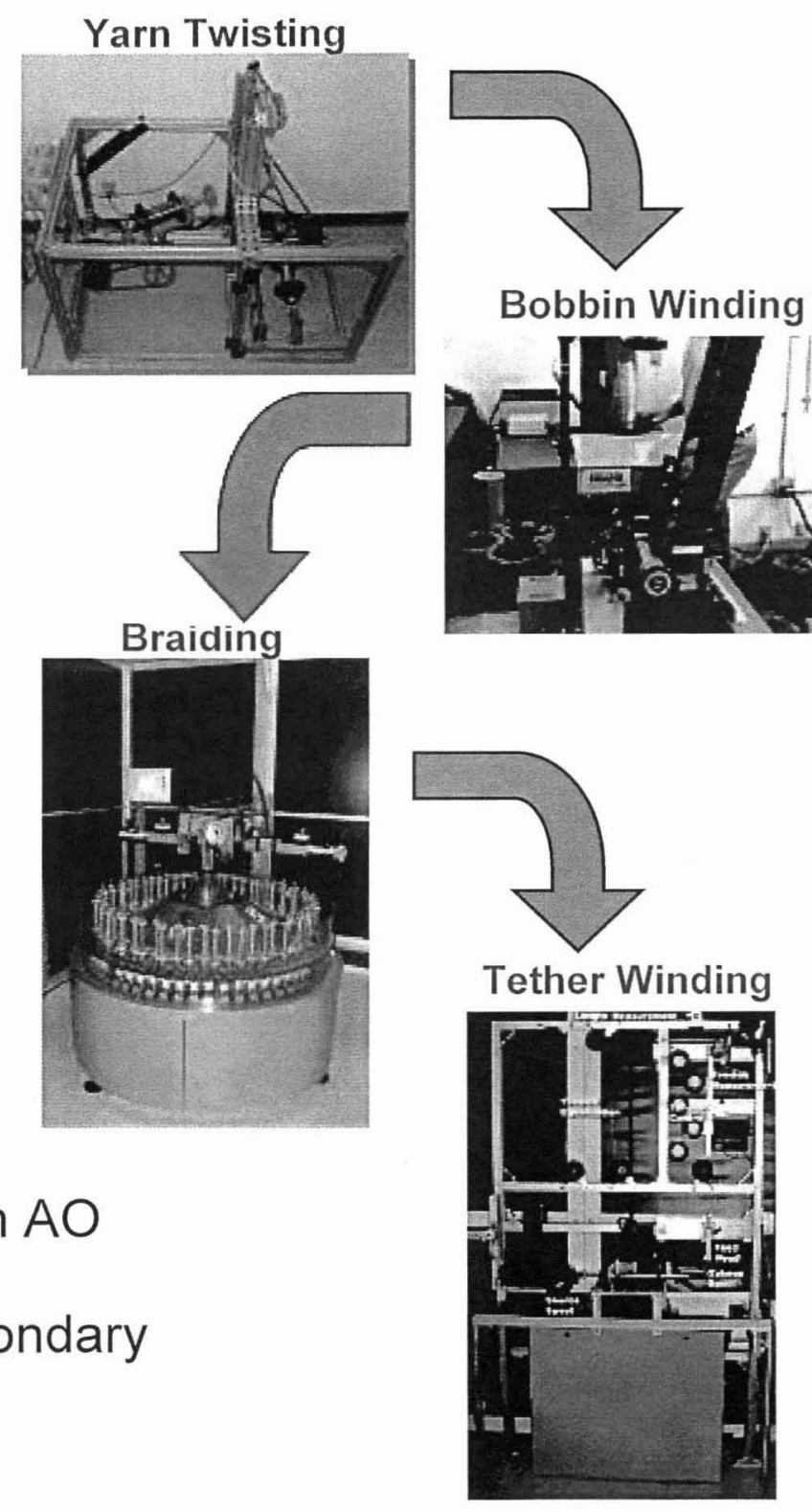




\section{Progress on Strength Tether Development}

- Developed multi-strand tether manufacturing processes

- Yarn plying/twisting

- Bobbin winding

- Braiding and splicing

- Spooling

- Quality control

- Developed three new braiding methods for fabrication of "integrated" tethers

- Cylindrical high-tenacity Hoytether ${ }^{\mathrm{TM}}$ is loosely interwoven with a cylindrical mesh conducting structure

- Proper interweaving binds the two structures together while enabling the tensile materials to carry the mechanical loads and preventing pinching/bending of yarns

- Developed commercial software tool for tether fabrication cost \& time estimations

- Produced numerous tether material samples \& full-scale

High-Strength Hoytether

Tri-axial conducting structure

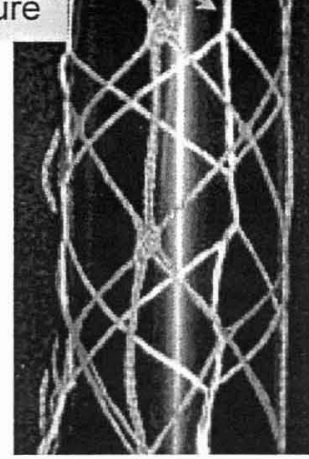

Integrated Hoytether ${ }^{\mathrm{TM}}$

\section{structures}

- Delivered five samples of yarn and braided materials treated with AOresistive coatings (Photosil ${ }^{\mathrm{TM}}$ and nickel) for testing in the NASA/MSFC AO facility.

- Tape and ribbon multi-strand tethers (Zylon® \& Spectra)

- Single-strand tether with predicted break-strength rating for MXER

- Three 140-meter continuous lengths of Hoytether ${ }^{\mathrm{TM}}$ prototypes:

- Zylon®, $1000 \mathrm{~kg}$ break strength

- Hybrid Tether, Zylon® $\mathrm{w} /$ twisted copper wire, $1000 \mathrm{~kg}$ strength \& $30 \Omega / \mathrm{km}$

- Spectra, $1000 \mathrm{~kg}$ break strength

- Manufactured a breadboard MXER Hoytether ${ }^{\mathrm{TM}}$ segment that is capable of

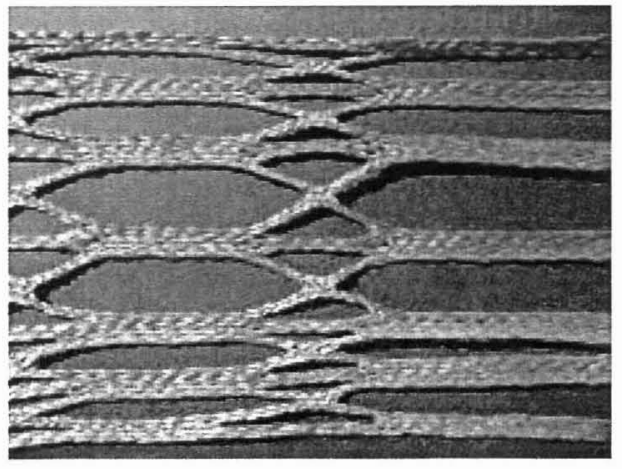

Hoytether ${ }^{\mathrm{TM}}$ Close-up carrying the required tensile load. 


\section{Progress on Strength Tether Coatings}

- Evaluated numerous tether coatings and/or process \{Goal of nominal 10-year orbital lifetime\}

- Triton atomic Oxygen Resistance (TOR) coating

- Polyhedral oligomeric silsesquioxane (POSS) process

- Photosil ${ }^{\mathrm{TM}}$ coating

- Metallized coatings

- Nickel

- Aluminum/alumina

- Investigated NextGen self-healing wire insulation technologies

- Investigated wrapping AO-resistant yarns on top of Zylon® yarn to provide an AO/UV resistant high-tenacity yarn with low mass impact

- Conducted initial mandrel bend tests on coated tethers

- Most material samples did not have bending issues

- Most tether samples experienced some coating removal

- Developed a cost database for coating tethers

- DRLI - PVD deposition coating

- SwRI - PVD deposition coating

- Triton - TOR coating

- Lockheed - Modified C-MAG PVD coating

- Numerous samples of tethers delivered to MSFC for testing in the MSFC Atomic Oxygen Facility (details on next slide).

- Now suggest a double costing process (before and after final structurally assembly) should be embraced with M5 rather than Zylon ${ }^{\circledR}$ as the strength polymer fiber.
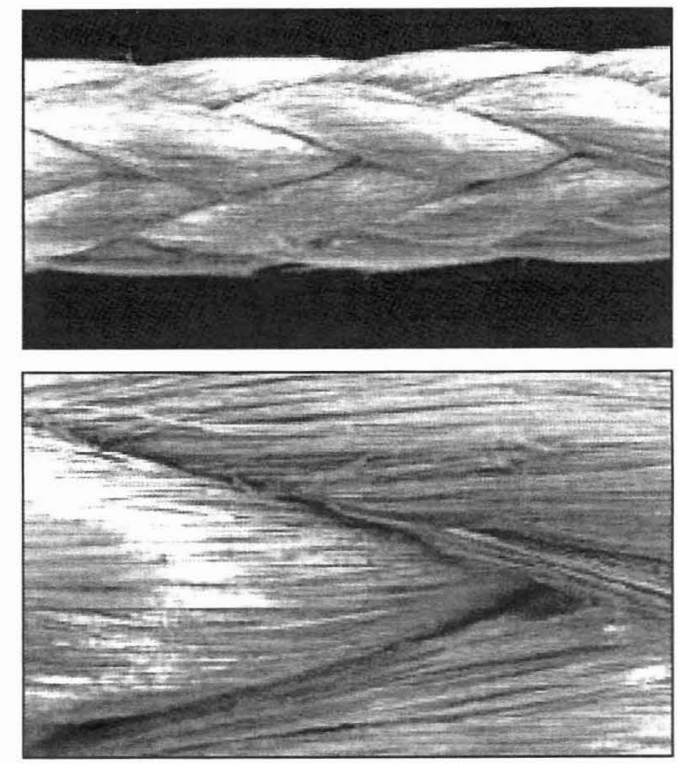

Lockheed Aluminum/alumina Coated Tether

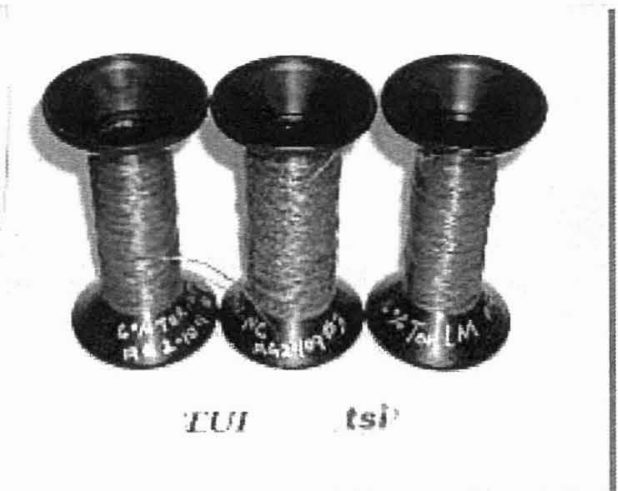

TOR coated Zylon ${ }^{\circledR}$ 


\section{Tether Testing and Analysis}

- Performed tensile strength testing of Hoytether ${ }^{\mathrm{TM}}$ structures at TUI

- Zylon $\circledast$ in a Hoytether ${ }^{\mathrm{TM}}$ configuration is consistently exceeding the theoretical tensile strength

- Initial tensile tests of Hoytether ${ }^{\mathrm{TM}}$ structures with cut lines

Structure is maintaining strength following several cuts

- Performed atomic oxygen (AO) and ultraviolet radiation (UV) testing on numerous samples provided by TUI and LMSS

- Photographed samples before and after exposure to simulated space environment, $\sim 3 \times 10^{21}$ atoms $/ \mathrm{cm}^{2}$.

- After simulated space exposure, the samples were weighed for mass loss, and suitable samples were mechanically tested to determine the tensile strength.

- Tethers samples supplied by TUI ranging from fifteen to twenty centimeters in length

- Spectra ${ }^{\circledR}$ and Zylon® coated with Photosil ${ }^{\mathrm{TM}}$

- Zylon® strand

- Metallized with nickel

- TSI'S TOR-LM $^{\mathrm{TM}}$

- Uncoated M5 fiber

- Mass loss and visual results of TUI samples

- Spectra and Zylon ${ }^{\circledR}$ tether materials coated with Photosil ${ }^{\mathrm{TM}}$ lost tensile strength without exposure to $A O$ and UV

- Nickel coating appeared to improve the strand's resistance to AO erosion

- Two nickel coated samples experienced mass gain suggesting that a nickel oxide layer had formed

Visually, the TOR-LM ${ }^{T M}$ coated Zylon® appeared to provide some protection from $A O$ and M5 with POSS was able to survive the testing regime without any apparent fraying or other damage

- The tensile test results were inconclusive, due to the handling difficulty with the strands.

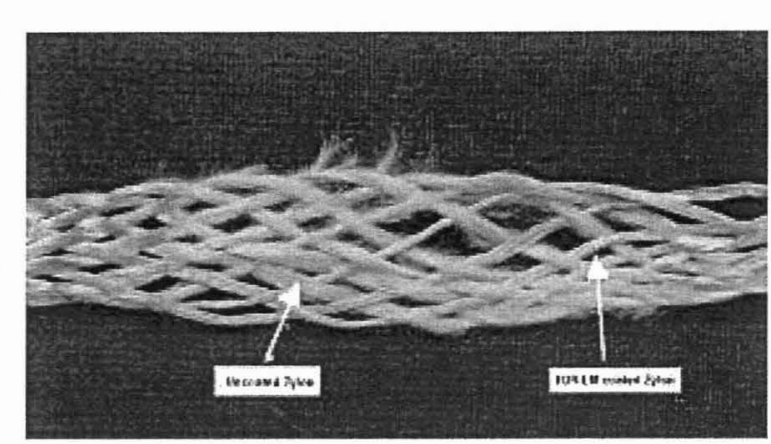

Zylon®-TOR-LM ${ }^{\mathrm{TM}}$ Hoytether after exposure

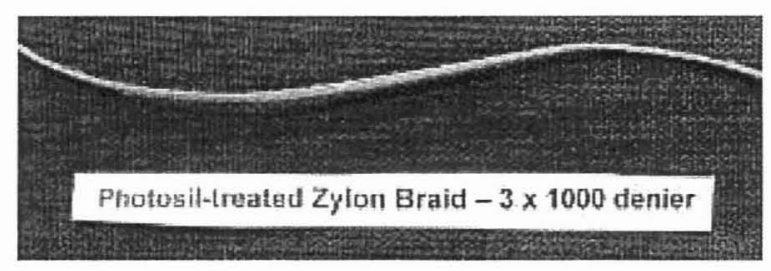

Zylon®-Photosil ${ }^{\mathrm{TM}}$ braid after exposure

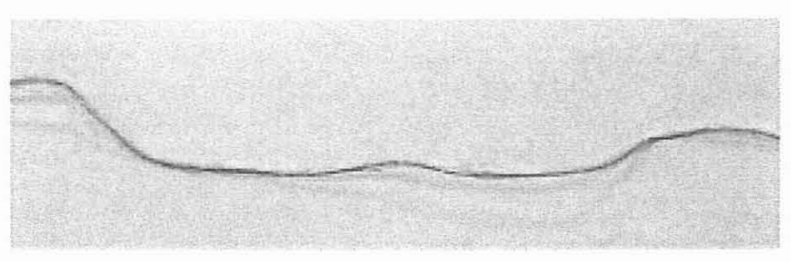

Zylon-POSS braid after exposure 


\section{Tether Testing and Analysis, con't.}

- Tether samples supplied by Lockheed Martin for AO/UV testing

- Uncoated Zylon®

- Zylon® braid

- Al PVD coating

- Zylon® braid with $\mathrm{Al} / \mathrm{Al}_{2} \mathrm{O}_{3}$ PVD coating

- Triton Systems TOR-LMTM coating

- Fiber coated POSSTM

- Zylon ${ }^{\circ}$ braid encapsulated within biocomponent Mylar/polyethylene facesheet

- with AI PVD coating

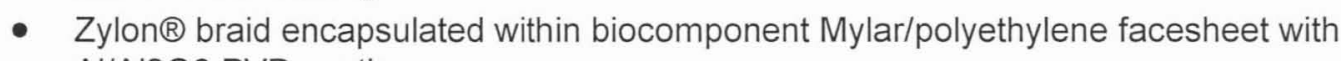
Al/Al2O3 PVD coating

- Samples were characterized by photography and mass change.

- No tensile testing was performed on the LMSS samples due to unavailability of reasonably sized, coated samples.

- Visual observations include

- Uncoated PBO braid with sizing showed very poor resistance to AO degradation

- Zylon braid

- Aluminum PVD coating showed poor resistance to AO erosion

- Aluminum and alumina PVD coating, had similar results to the previous sample

- TOR-LMTM coating was slightly bleached by $A O$ whereas the fibers did not pull free

- TOR-NCTM coating, was very similar to the previous sample, though not as much bleaching.

- Both encapsulated (facesheets) PBO braids were basically unchanged by AO exposure

- The mass change for these samples was not severe

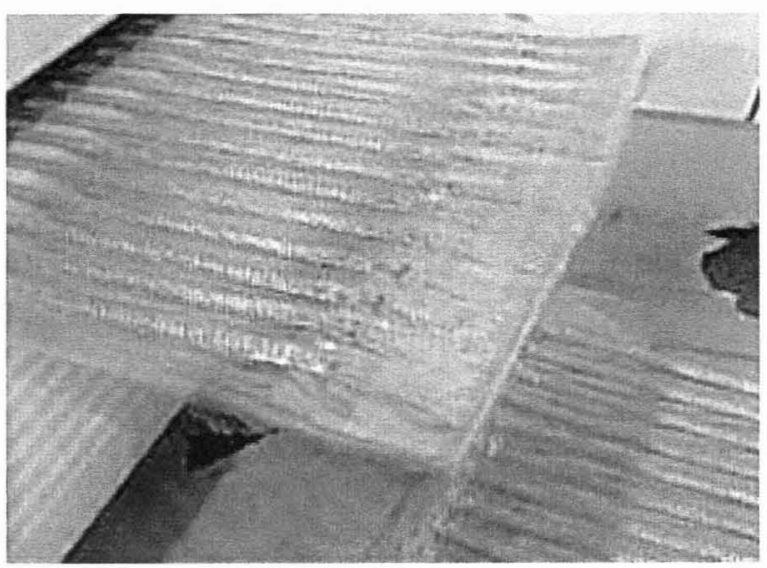

Manufacturing of Zylon braid encapsulated within Mylar/polyethylene facesheet

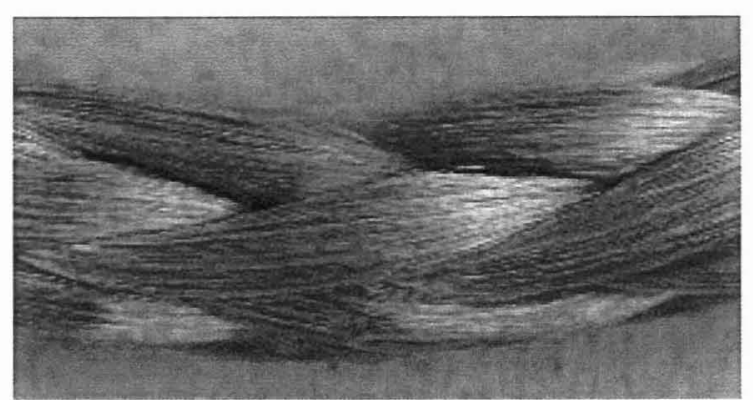

Zylon-PVD $\mathrm{Al} / \mathrm{Al}_{2} \mathrm{O}_{3}$-coated at strand then braid

1 um Nickel an 250 denier Zylon

Zylon-Nickel Strand after exposure 


\section{Propagator/Predictor Code Development}

STAR, TTU, and TUI

- MXER Tether's configuration makes rendezvous extremely difficult

- $\sim 100 \mathrm{~km}$ long tether

- Spinning dynamic structure

- Tether tip and payload position accuracy

- Must be known with extreme accuracy (within meters)

- Forward position prediction

- Must know approximate tether tip position one orbit prior

- Must pre-position payload in precise orbit to match timing

- Range-sensing system provides final position/timing corrections

- Cycle II NRA Propagator/Predictor Code Focus

- Determine if accurate propagation/prediction of a spinning dynamic structure is feasible

- Develop a tether propagation algorithm with precise accuracy

- Develop a design code to conduct trades on different tether configurations 


\section{Progress on Propagator/Predictor Code Development}

- Two different approaches taken:

- STAR focused on developing a tether propagation algorithm that could achieve the desired accuracy yet run extremely fast on a computational platform.

- Applied traditional, analytic mathematical techniques to the tether model that allowed orders-of-magnitude improvements in computational speed.

- Achieved the desired accuracy in a model that included the full spectrum of idealized tether dynamic effects and external gravitation.

- Later addressed additional forces

- Drag and solar radiation pressure

- Realistic tether materials effects

» Material non-uniformity

» Creep

"Mass loss due to erosion

- Simple addition of a laser ranging system between the deployer reel and the tether tip could allow the tether to adjust its length slightly and achieve the required accuracy

- TTU pursued modeling techniques that were more computationally intensive, yet led to extremely accurate solutions.

- Results were used to "benchmark" the accuracy of STAR's results

- In both cases, the remarkable results of these activities gave the program a dramatic increase in confidence that successful rendezvous could actually be achieved at realistic speeds and with straightforward operational techniques.

- Original estimate was $\$ 2 \mathrm{M}+$ for code version 1.0 and most likely it would take 3 full code projects to get to an accuracy of $\sim 10$ to 100 meters

- Accomplished under $\$ 1 \mathrm{M}$ and produced an accuracy meters with less computational power

- Substantially less time \& cost when compared a trajectory code development program 


\section{Catch Mechanism Development}

LMSS and TTU

- Prediction of the error window is ellipse-like with defined major and minor axes.

- Nearly one-hundred concepts were generated and categorized

- Following an AHP (analytic hierarchy process), only a small number of concepts remained for further evaluation

- TTU chose and manufactured a single concept, called the "QuadTrap"

- LMSS fabricated two concepts: the "umbrella" and the "PatTrap"

- QuadTrap Description

- Consists of four slender beams and four corner nodes connected with eight prismatic joints, all arranged symmetrically.

- The catch mechanism begins passive closure when the payload boom penetrates the horizontal aperture of the quad-trap.

- For release, the payload's boom simply reverses the mechanical lever which allows the boom to slide out of the catch mechanism.

- Three significant features of the QuadTrap are as follows

- Matches estimated error ellipse in an efficient fashion

- Allows for passive actuation during closure

- Passes all significant loads in tension through cable members.

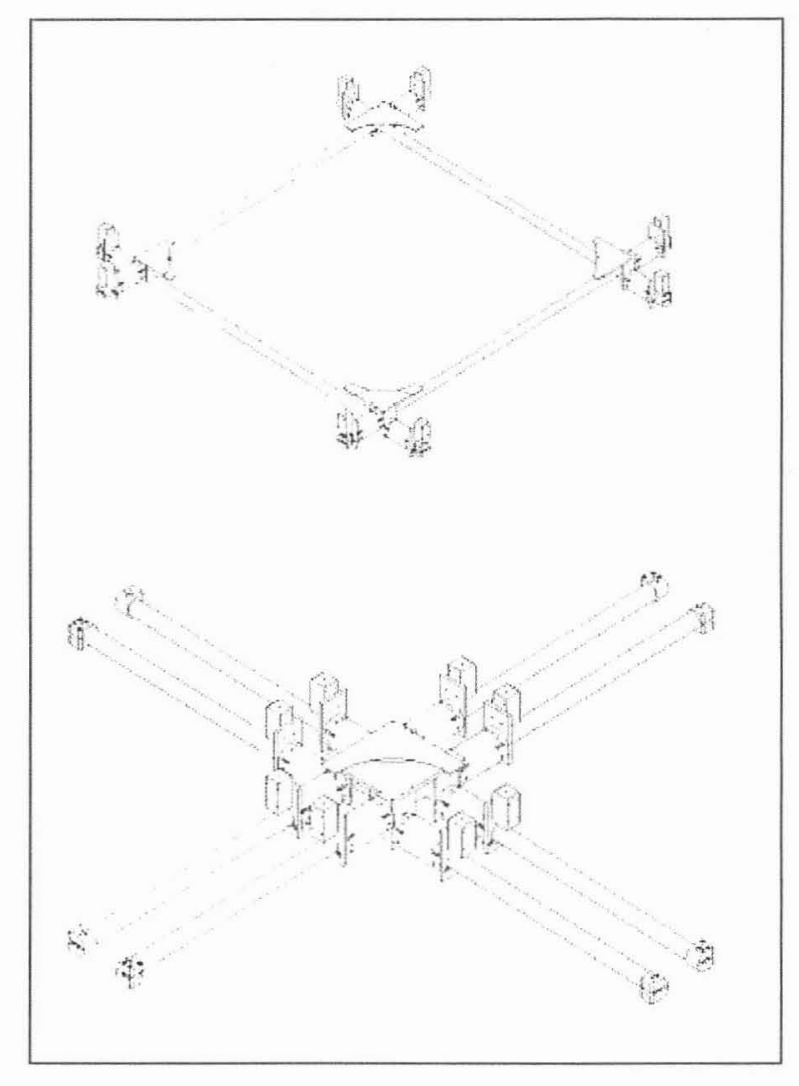

Schematic of QuadTrap

A) Open configuration

B) Closed configuration 


\section{Catch Mechanism Development}

\section{LMSS and TTU}

- PatTrap Description

- Consists of a circular ring structure with four semi-circular bands (two sets)

- Each set contains two bands - connected to a single motor mount at each endpoint.

- The catch mechanism begins closure when the payload boom penetrates the horizontal aperture of the PatTrap and the two motors close each band set around the payload boom - forcing the payload to the center of the aperture

- The release is the same as the QuadTrap

\section{- Umbrella Description}

- Consists of square structure with "grab lines" strung from each side (similar to the strings on a tennis racket).

- The prototype was tested and the concept has three unique advantages

1. No moving parts on the MXER side of the system, where repair would be extremely difficult

2. No active sensing of payload/boom 'presence'

3. The ability to accommodate the full 100 meter overtravel without colliding with the payload or having an impact load when the "slack" is taken up.

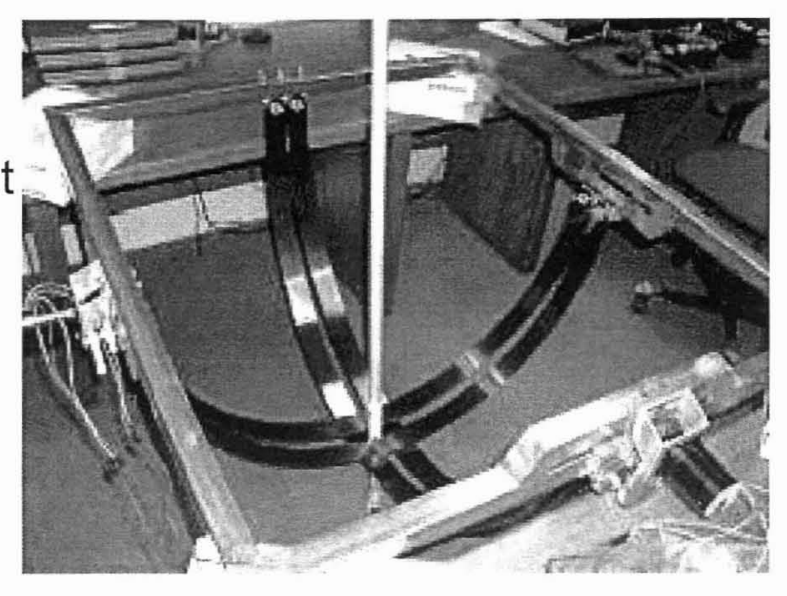

Mock-up of PatTrap

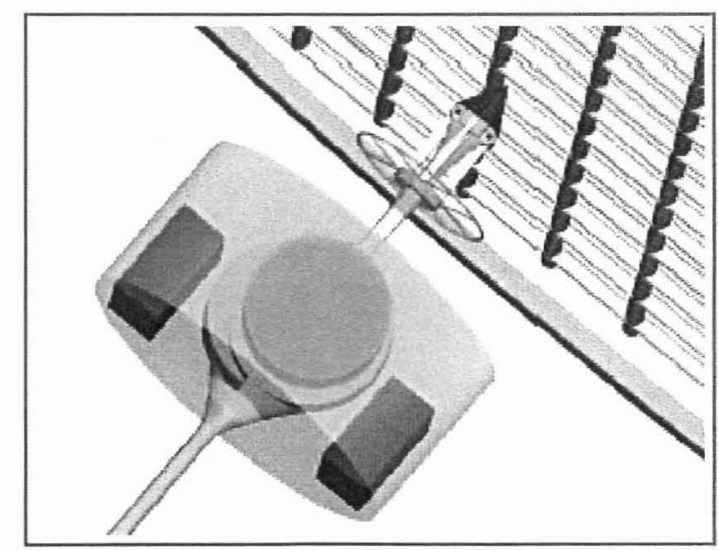

CAD Drawing of Umbrella and Probe 


\section{Catch Mechanism Testing}

- All three catch-mechanism engineering-models were evaluated in the TN Tech High Bay Facility in Cookeville, TN.

- The setup included a payload launch system using hydraulics to vertically launch a platform that would throw a "dummy" payload towards the catch mechanism.

- The PatTrap capture system was designed and tested at LMSS and shipped to TN Tech for further evaluation.

- Consequently, this design did not have the option for a test, evaluation, and alteration loop to optimize the design.

- As a result, the PatTrap system did not perform well due to high costs prohibiting the purchase of appropriately-sized motors to drive the circular rings.

- The QuadTrap performed on the order of several hundred simulated catches while capturing approximately $>90 \%$ of the time.

- TN Tech also incorporated accelerometers and automated the capture sequence with ranging devices.

- After the incorporation of the automated catch actuation, the QuadTrap laboratory hardware successfully captured the payload in $100 \%$ of the attempts

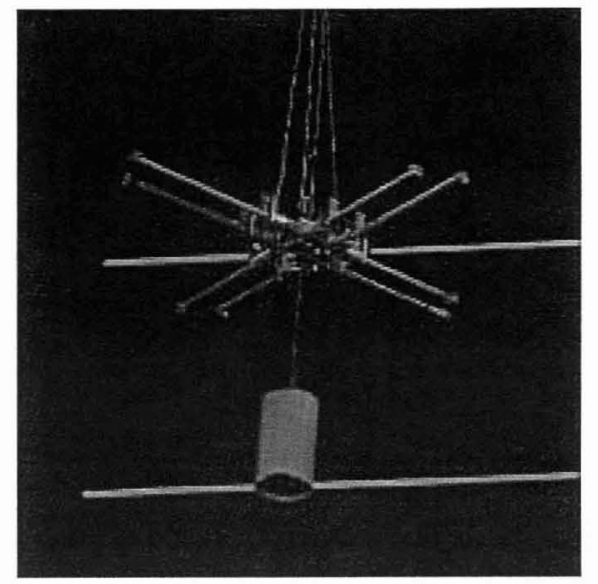

Successful Catch with QuadTrap

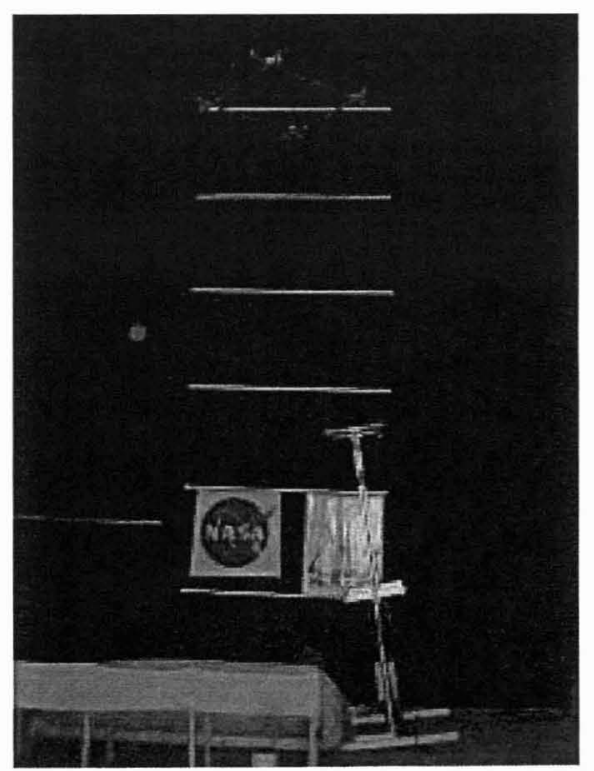

Test Setup 


\section{Catch Mechanism Testing}

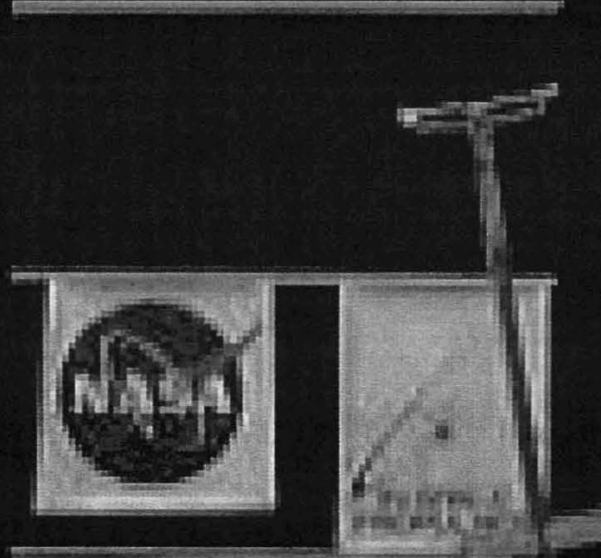




\section{Plasma Contactor Development}

MSFC, SRS and JPL

- A Phase II SBIR (SRS Technologies) task focused on the feasibility and development of a Gridsphere Anode for electrodynamic tethers.

- The grid-sphere concept includes a spherical, metallic grid that incorporates an outer layer of photolyzable material.

- Once deployed in space by gas pressure, the outer "balloon" material will photolyze, or melt away, leaving only the metallic grid structure remaining.

- The grid would then, with voltage applied, act as a spherical anode collecting electrons.

- SRS designed and built a 5-m prototypical grid-sphere.

- The plans included performing an inflation test and repackaging this $5-\mathrm{m}$ prototype into a canister for future inflation test inside vacuum.

- However, during the manufacturing of the grid-sphere, the lights in the assembly area photolyzed the outer material enough that the material became embrittled with tiny holes.

- The inflation test worked well, but the grid-sphere was rolled into a container instead of the pre-planned folding process and stowed with further testing cancelled.

- In-house MSFC activities computationally investigated the following electron collectors:

- The bare wire tether

- The tape wire configuration

- The end-body (solid and grid-sphere)

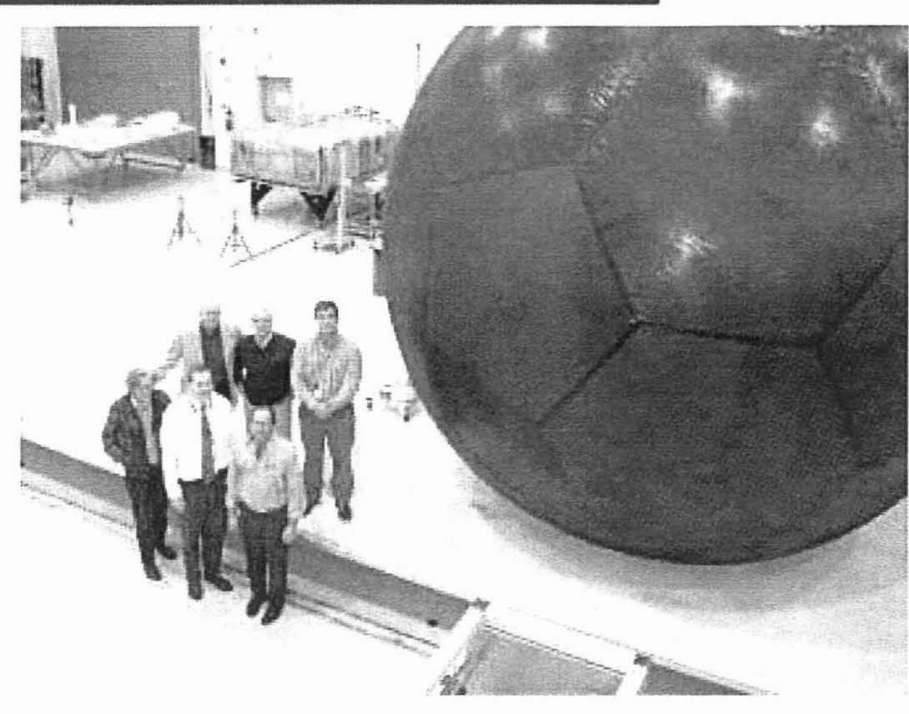

Inflated 5-m Grid-Sphere

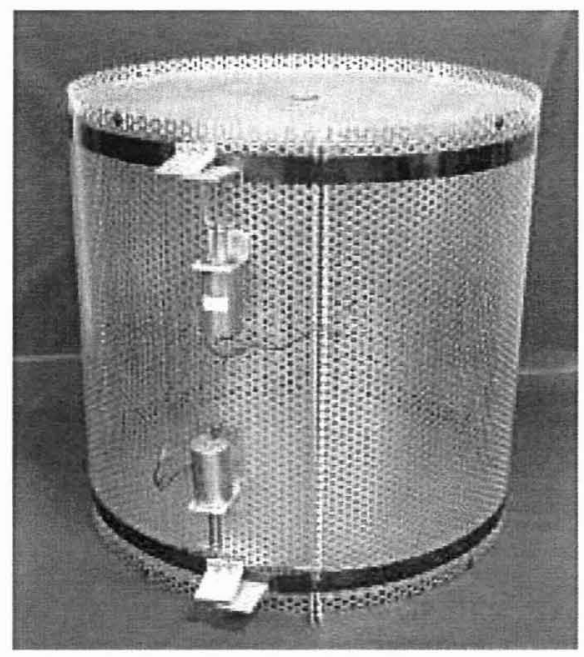

Grid-Sphere Stowage Canister 


\section{Plasma Contactor Development}

MSFC, SRS and JPL

- A second Phase II SBIR (SRS Technologies) focused on the development of the Solid Expellant Plasma Source/Contactor (SOLEX)

- The SOLEX will provide an efficient, robust and low-mass plasma generator for several applications including, spacecraft charge control, electrodynamic tethers and electric propulsion thrusters.

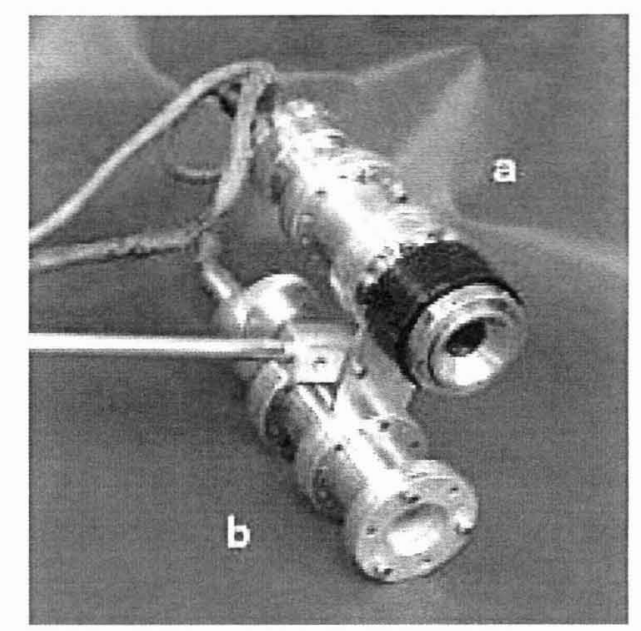

- During this time, an independent physics review was held to consider plasma collection options and its implication on MXER.

- Their findings substantiated the SOLEX-like approach and practicality of MXER operations from a fundamental physics viewpoint.

- An early Directed Task was given to JPL to complete FEAC testing of advanced solid-state plasma contactors

Prototype SOLEX Assembly

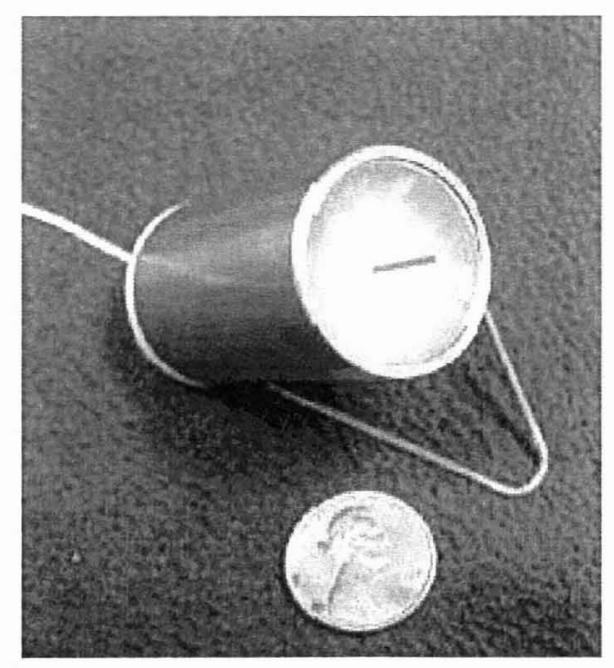

SOLEX Preliminary Test Unit 


\section{Electrodynamic Tether Development}

Orbitec and TSI

- A Phase II STTR centered on the development of technologies and analysis of a tethered flight experiment called the Tether Electrodynamic Spinup and Survivability Experiment (TESSX).

- TESSX is designed for space spin-up in order to generate centripetal forces and create an artificial gravity environment.

- TESSX consists of two end-masses connected by a tether

- Spin was originally to be created by electrodynamic forces acting on the tether.

- Trade studies indicated that modifying the spin rate through variable tether lengths is most attractive

- Flight experiment designs and several potential target customers were developed

- A Phase II SBIR task was awarded to Triton Systems for the advancement of coatings onto polymer fibers.

- Triton Systems has been developing the process for applying and evaluating lightweight metallic layers to the surface of high performance synthetic fibers for use as conductive tethers.

- Auburn University is continuing hybrid tether development and producing prototype conducing tethers

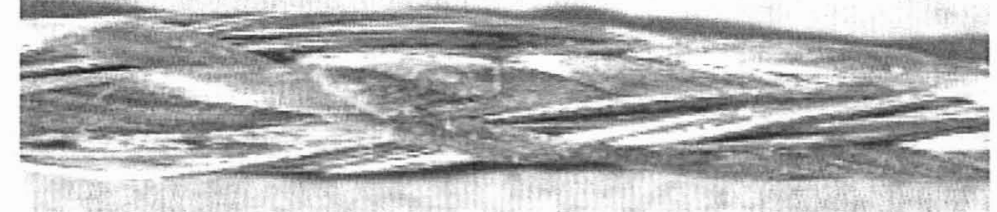

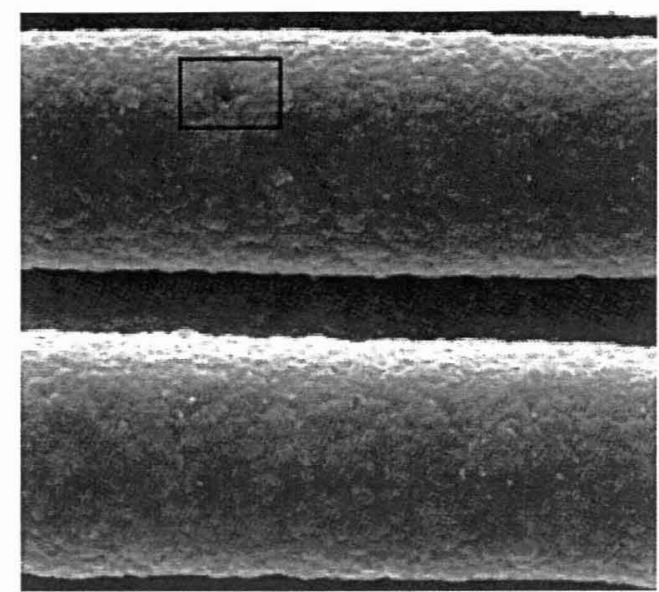

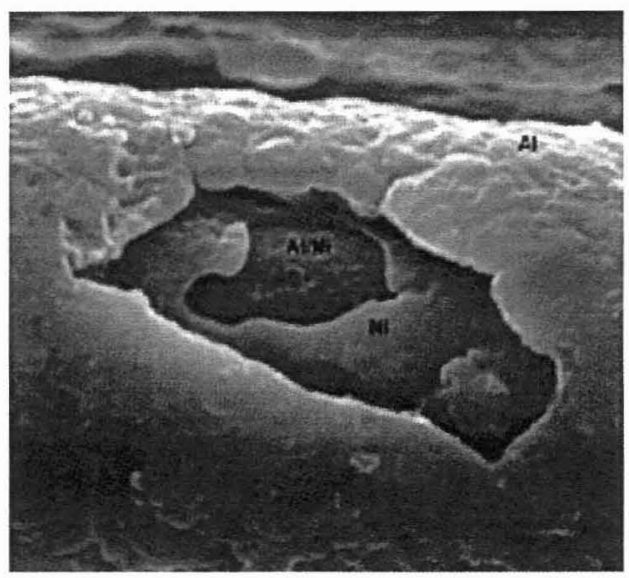

Scanning Electron Microscope pictures of Aluminum Plated onto Electroless Nickel Zylon Fiber 


\section{Deployer \& Mechanism(s) Development}

- Under a Phase II SBIR, TUI researched and analyzed the overall MXER design while focusing on developing a MXER-like deployer mechanism.

- Another area of interest in this SBIR was the analysis of a tether systems interaction with the catalogue of existing space debris/objects.

- Additional development has taken place under the TTU Cycle II task that included mechanism development for the MXER catch mechanism.

- Initial investigation into a spatial joint, gimballing mechanism, for the complementary component to the catch mechanism, the payload boom.

- This mechanism, conceptually, could incorporate this gimbal to point the boom into the catch aperture for capture.

- The "Canfield Joint" is also key to solar panel tracking on a rotating platform such as MXER (this problem is found in a host of space operations for almost every spacecraft missions)

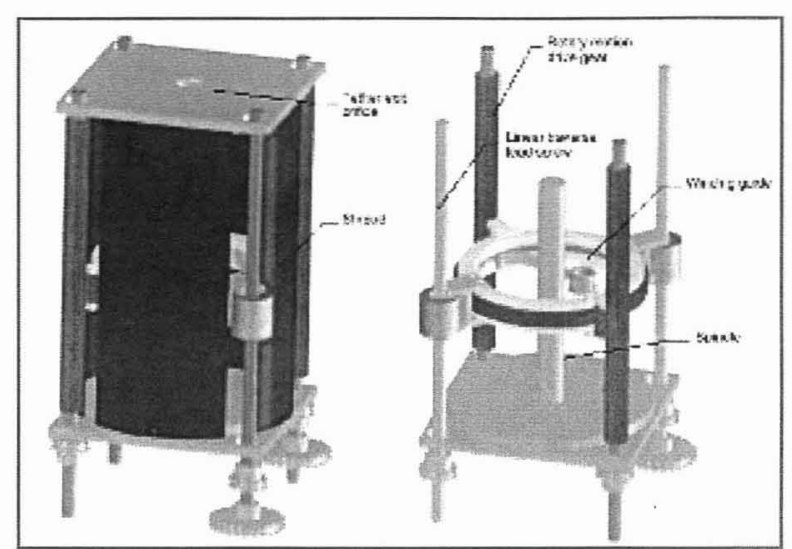

MXER Deployer Concept
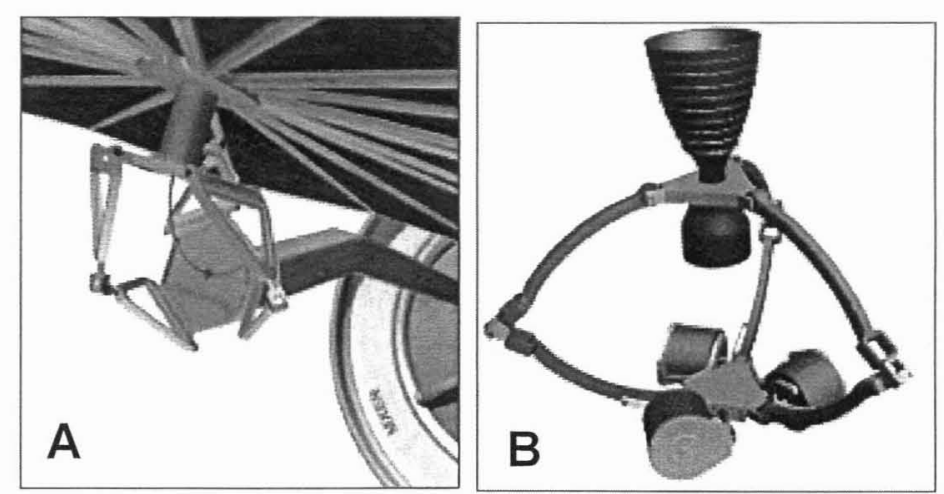

"Canfield Joint"

A) Mounted with solar array

B) Mounted with RCS thruster 


\section{Other MXER Activities}

- MSFC Independence Peer Review

- Lockheed Martin MXER System Cost Driver Study

- Independent Demonstration Mission Recommendation Study (Lockheed Martin)

- SAIC MXER Economic Study

- Three yearly MXER Technology Assessment Group (TAG) meetings

- Graduate student research on dynamic input-shaping (GA Tech)

- MAST Flight Experiment

- Vacuum-field Electrodynamic Propulsion Independent Review (VEPIR) 


\section{MXER Project Conclusions}

Maintained focus on MXER's economic excellence

- No technology for technology sake

- In $\sim 3$ years a total of $\sim \$ 5$ million (including funding outside ISPT) was invested in MXER and its related technologies

- Retired most major technology objection or "tall pole"

- Did not finish survivable tether design but objective of cost-effective manufacturability was established (note: all contract work is not complete).

- Exceeded all expectation in catch mechanism and computational propagators

- The recent development raised the Technology Readiness Level (TRL) of subsystems as much as 3 or 4 levels.

- There are now established resources that can address any MXER tether type application from continued systems development, to small flight demonstrations, up to a modest-sized operational system

- Few programs have achieved the high level of technical success, within the given budgetary constraints, as this MXER tether project 


\section{Questions}

\section{Famous Military General Quotes... I thought you said Jokes!}

\section{"You've seen a general inspecting troops before haven't you? Just walk slow, act dumb and look stupid!"




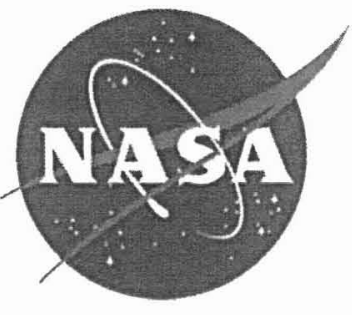

www.nasa.gov 


\section{Backup Slides}




\section{MXER Tether Design Genealogy}
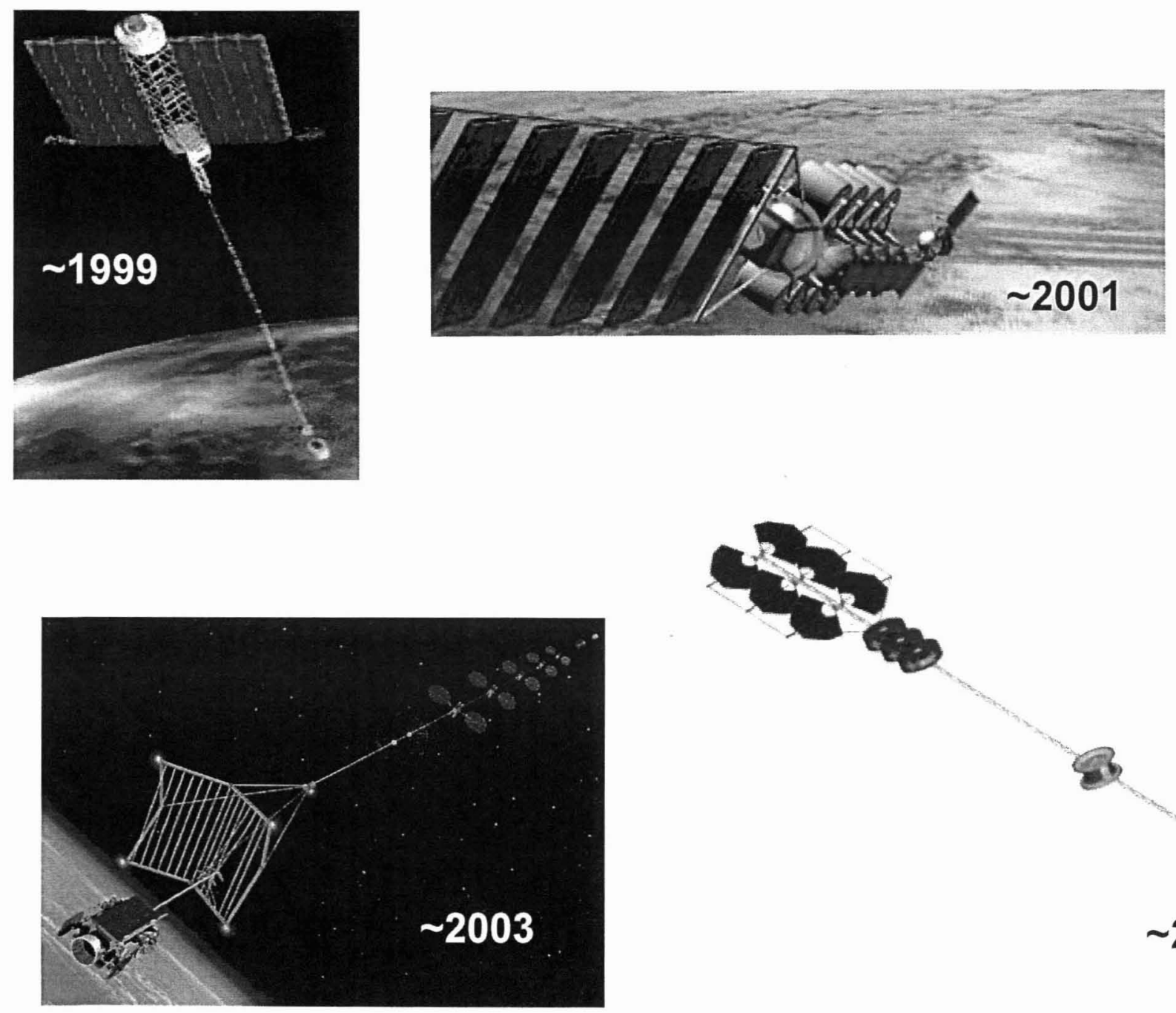

Launch Options

- Single launch

- Multiple launches

$\checkmark 100 \mathrm{~kg}$ to $5000 \mathrm{~kg}$ payloads

- Catch opportunities

- Multiple catches (equatorial orbit)

- Single catch (nonequatorial orbit)

- Ballast mast options

- Efficient use of upper stage

- Discard upper stage

- Power nodes

- Distributed

- Confined

$\sim 2005$ 


\section{MXER's Solar Array Genealogy}
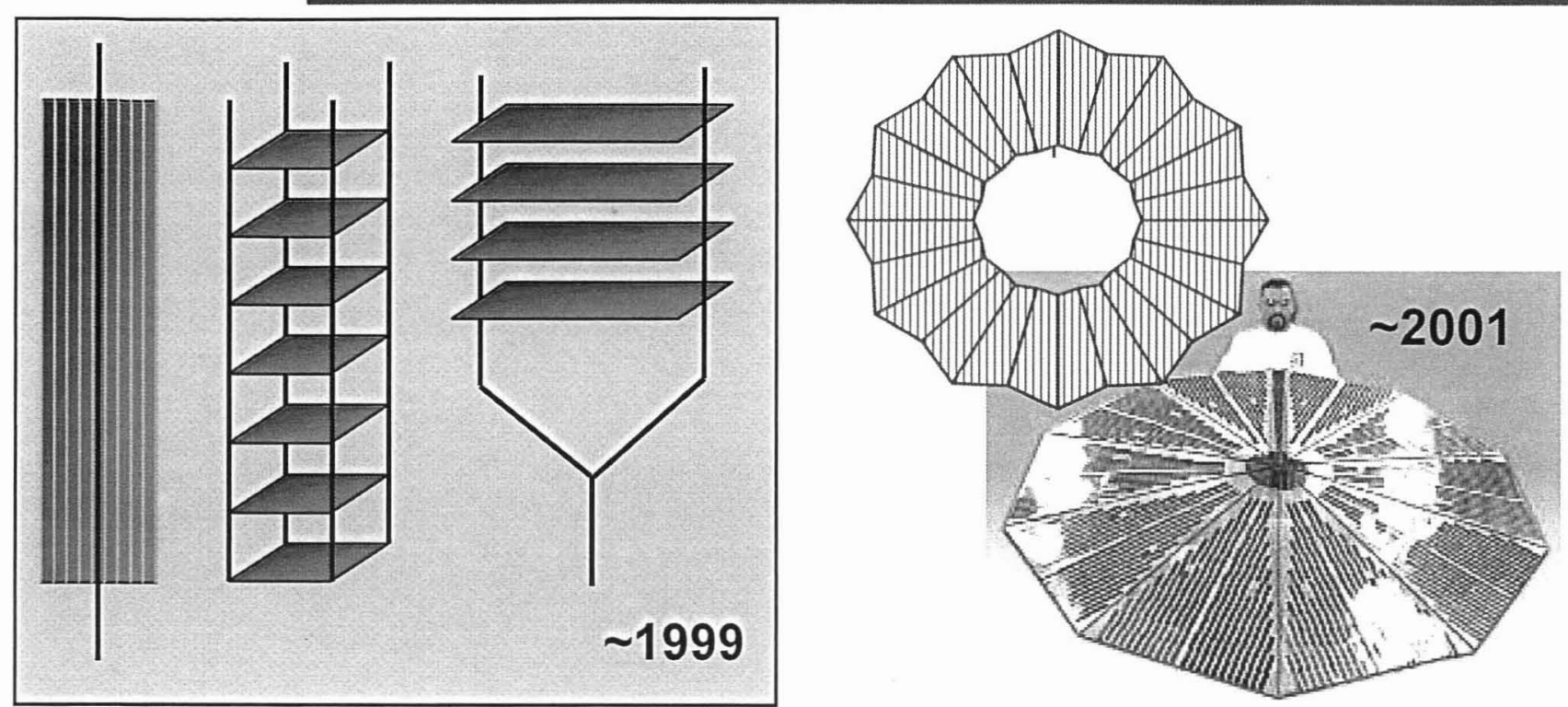

- Solar array shape options for optimal stowage

- Body-mounted

- Venetian blinds

- Square shaped

- Round shaped

- Fan-dance

- Folding petals

$\checkmark 9 \%-35 \%$ efficient solar cells

$\checkmark$ Canfield Joint
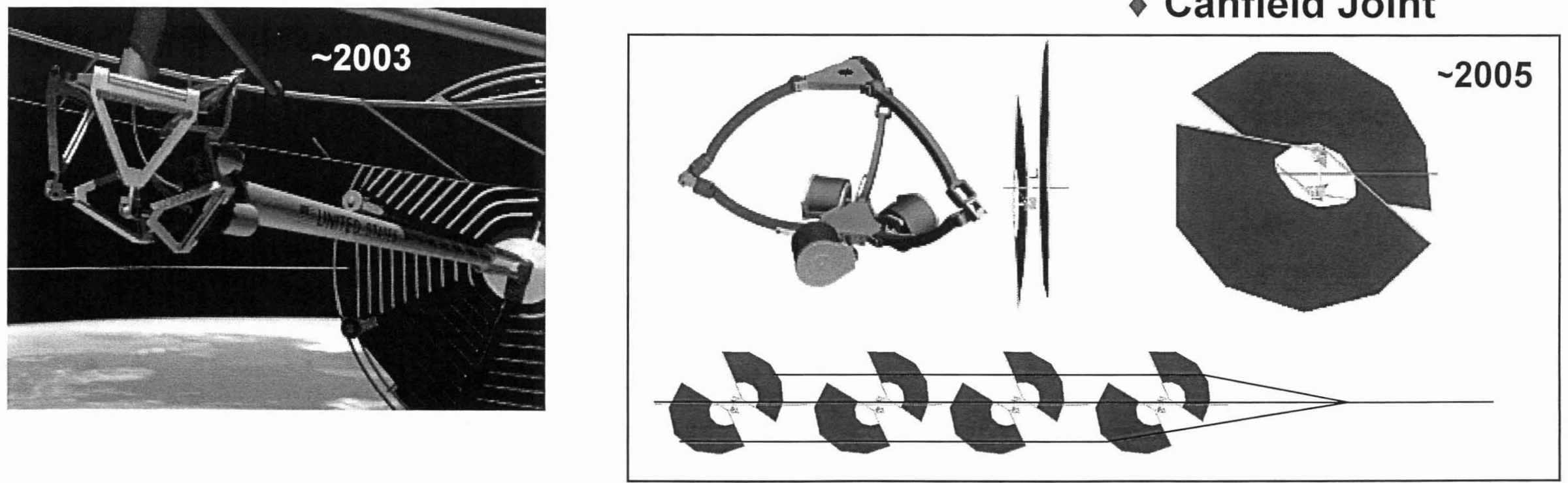


\section{MXER Power System Genealogy}
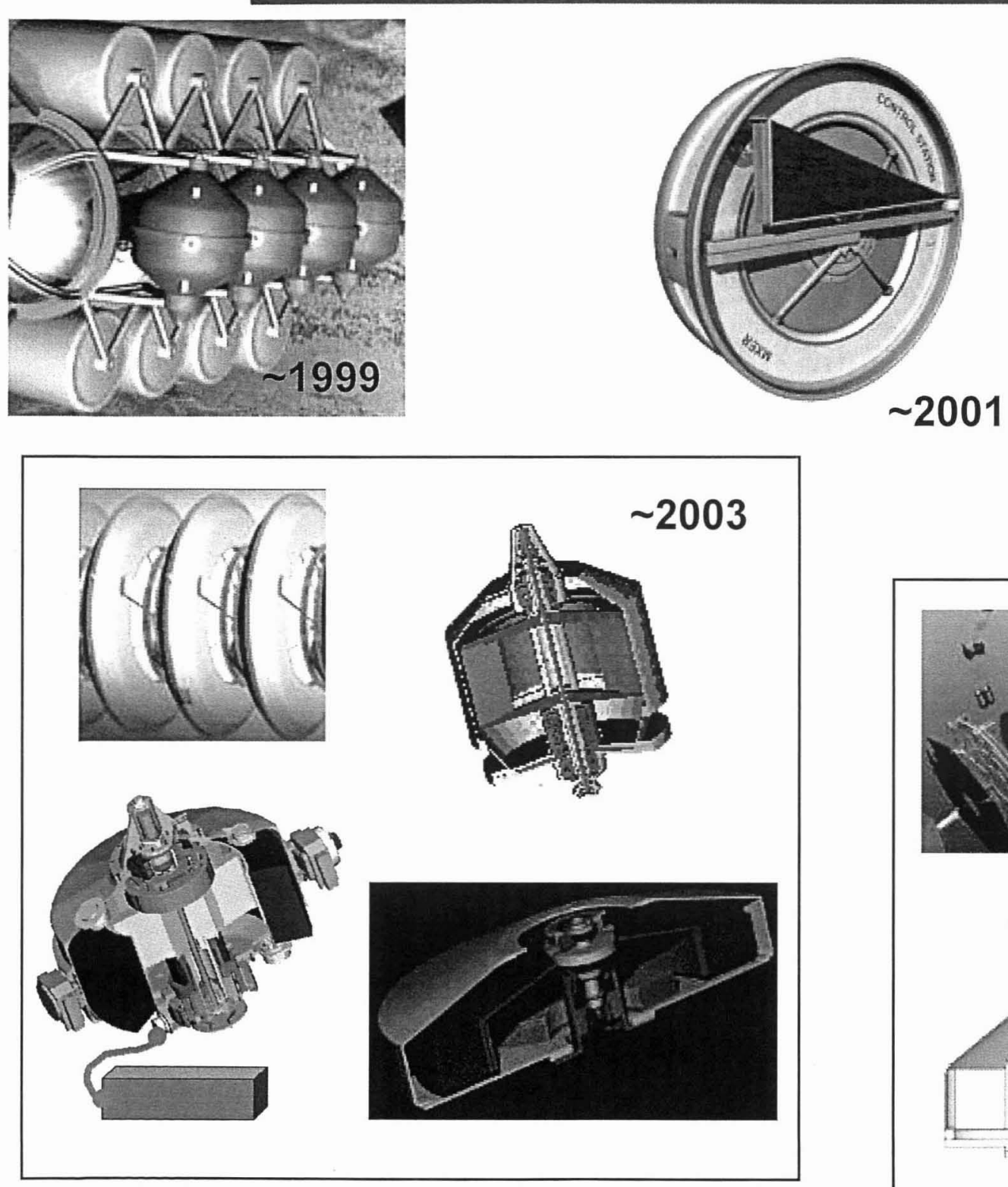

- Direct Drive

- Batteries vs. flywheels vs. capacitors

- Single Power Station

- Multi-node 'Satellites'

- Large to small diameter wheels
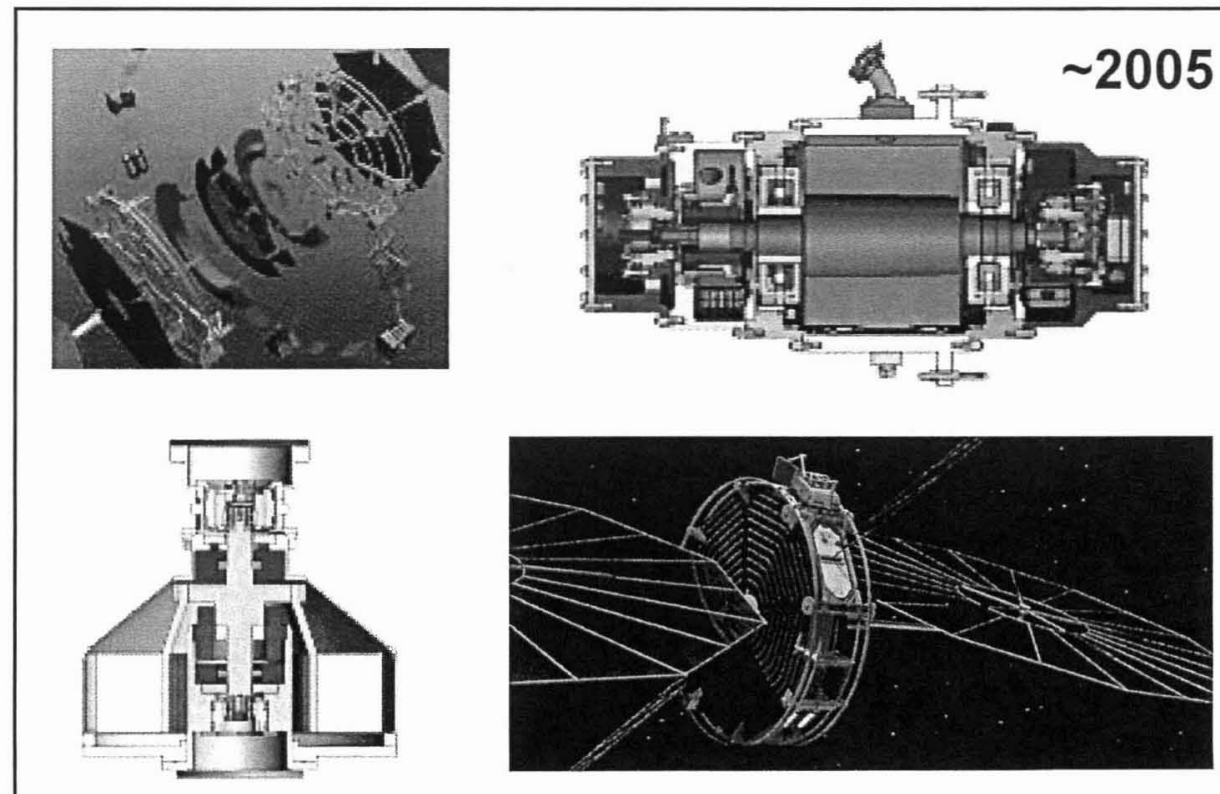


\section{MXER Plasma Contactor Genealogy}

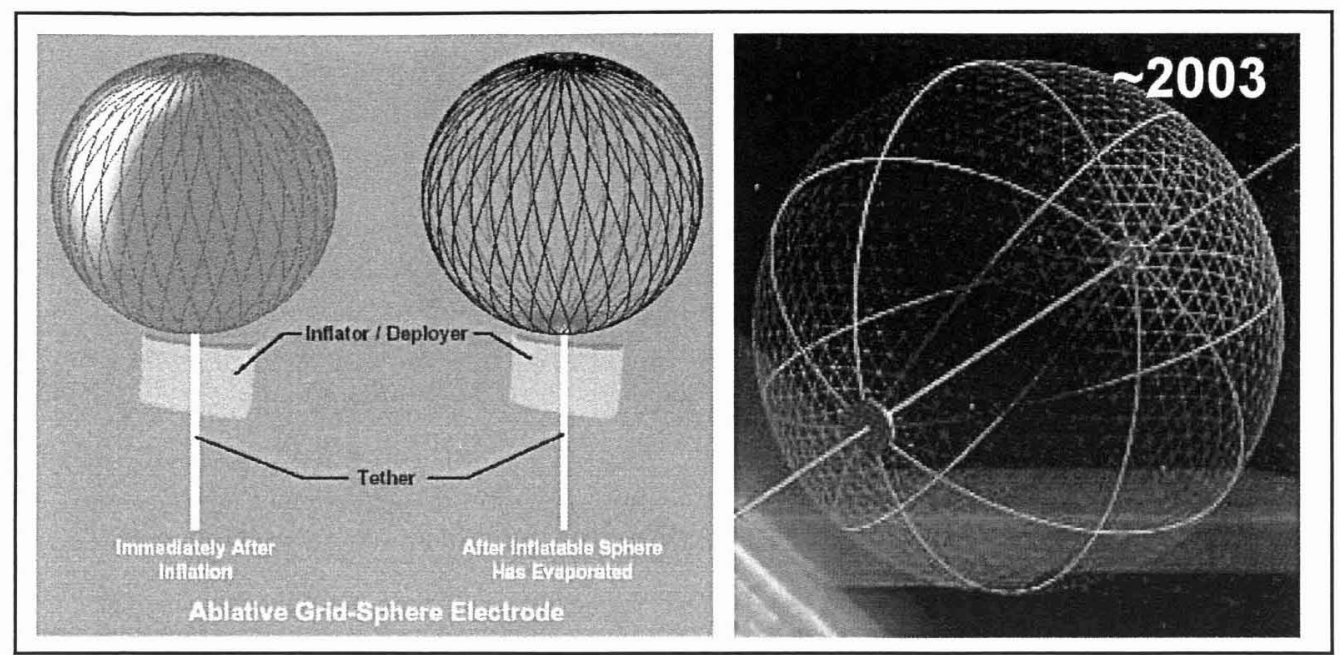

- Bare wire tether

- Grid Sphere

- Ribbons

- Field Emission Array Cathode (FEAC)

- SOLEX
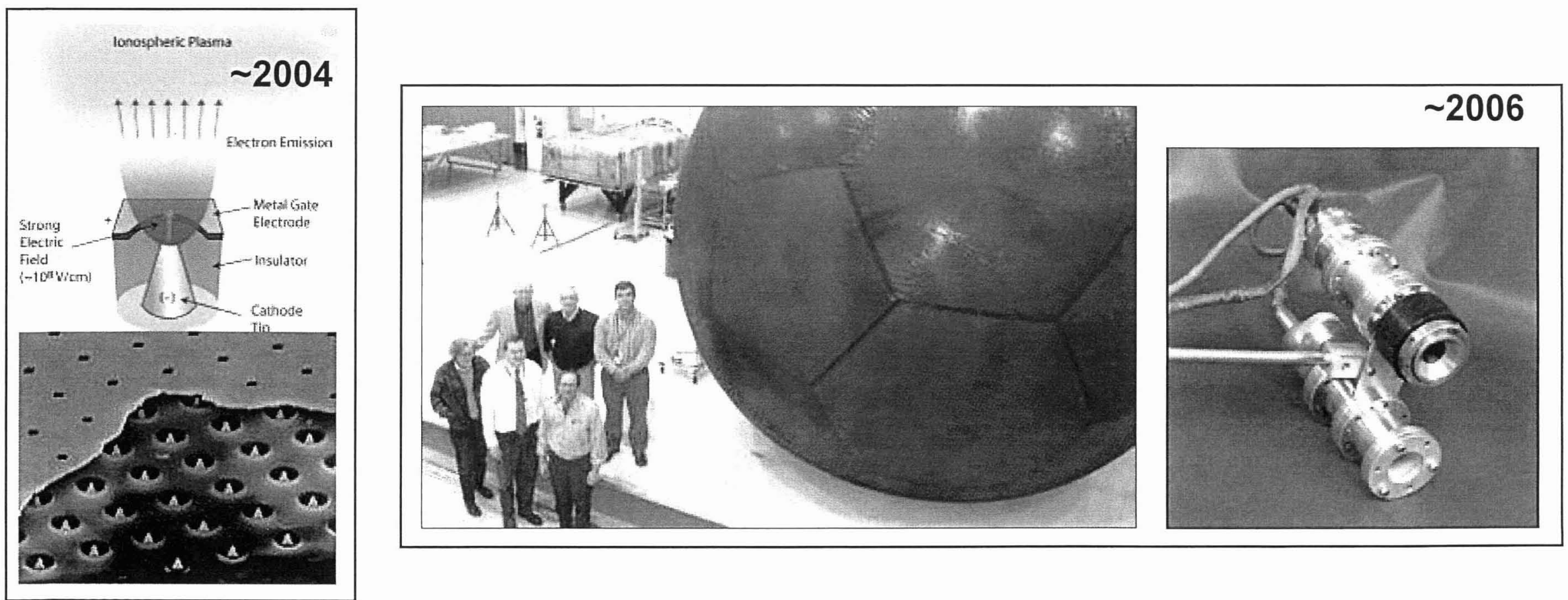


\section{MXER Catch \& Release Mechanism Genealogy}
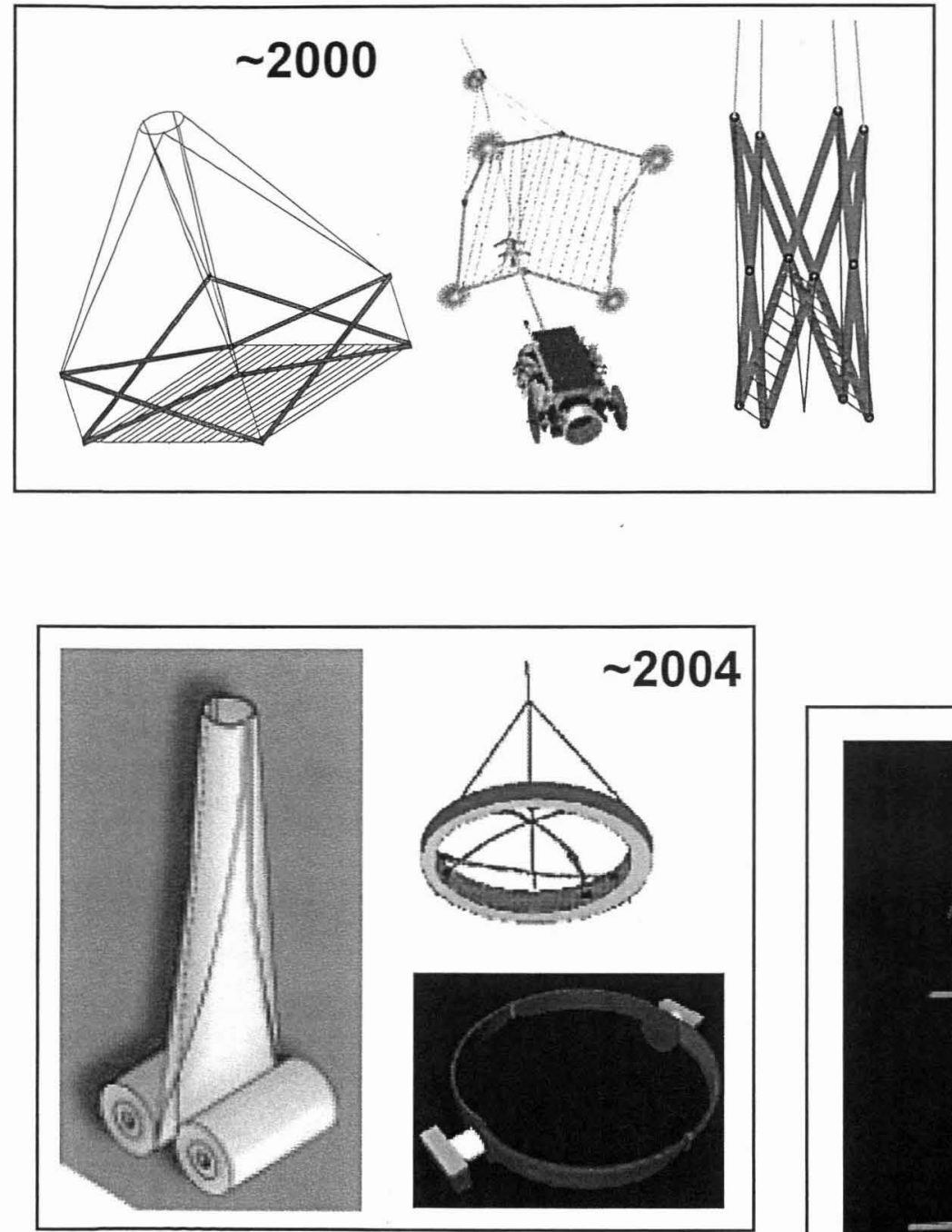

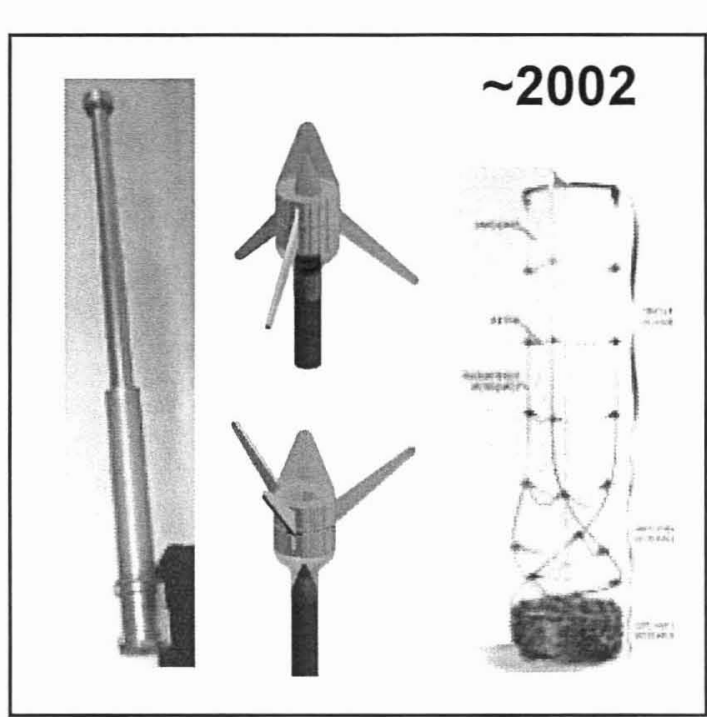

$\checkmark$ Unknown

$\checkmark$ Rope mesh

$\checkmark$ Collapsing mesh

- Tenn. Tech \& Lockheed's ideas

- Bear-Trap

Quad-Trap

Bi-stem boom

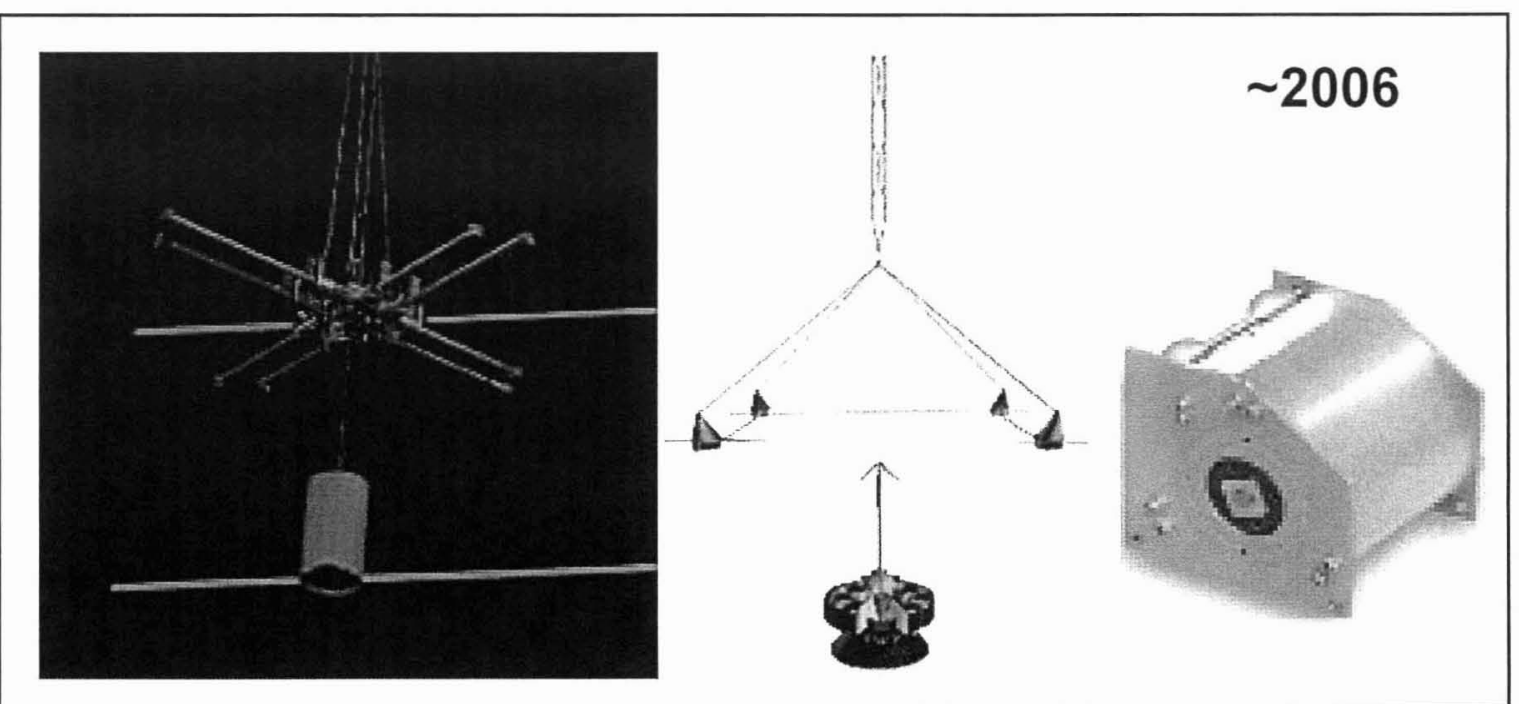




\section{MXER Launch \& Operations Genealogy}

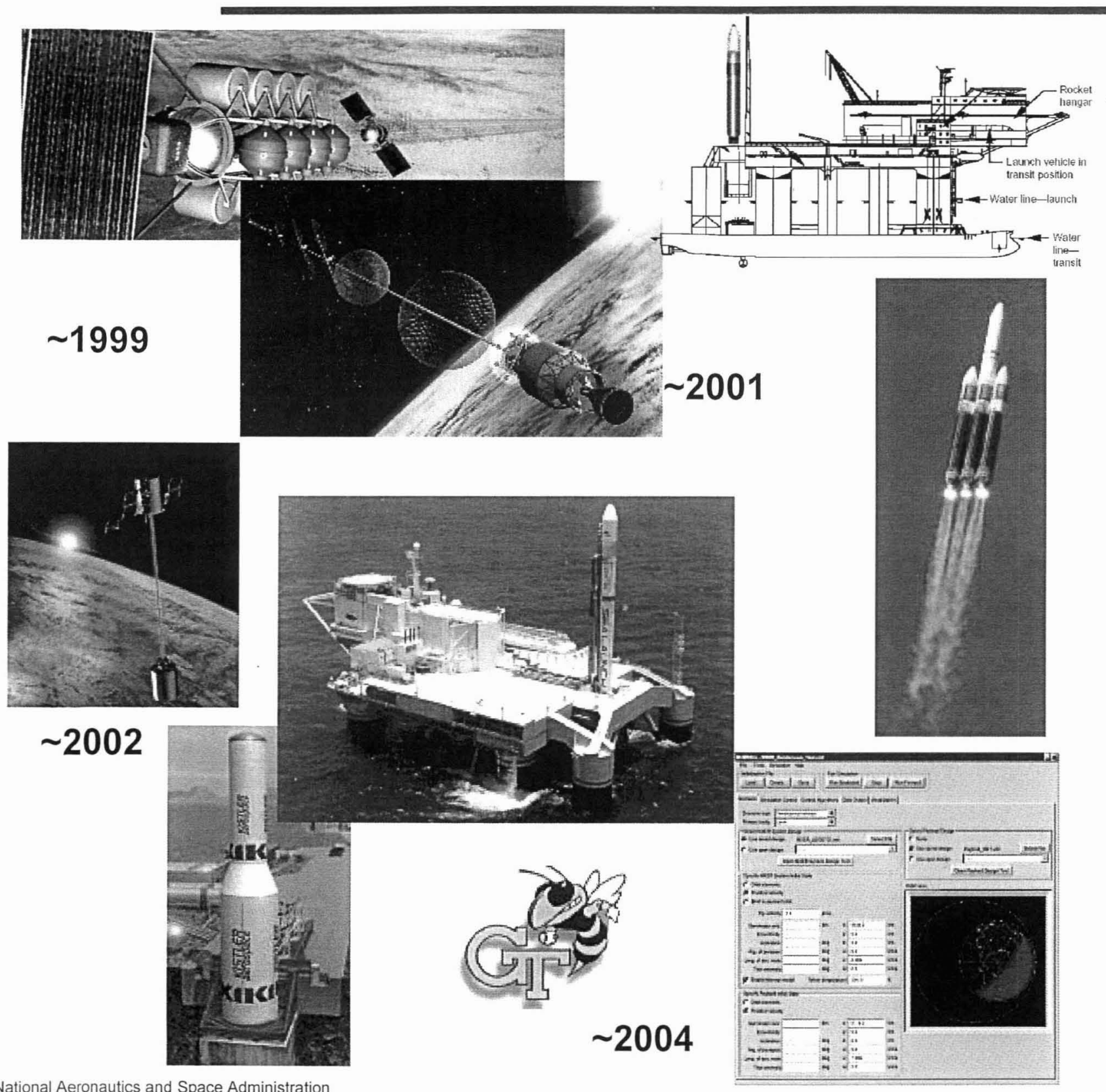

- Equatorial launch

- Non-equatorial launch

- Sea-Launch

- Kistler

$\checkmark$ Space X

- Delta IV

- Supercomputer predictors

- CM accuracy with active control

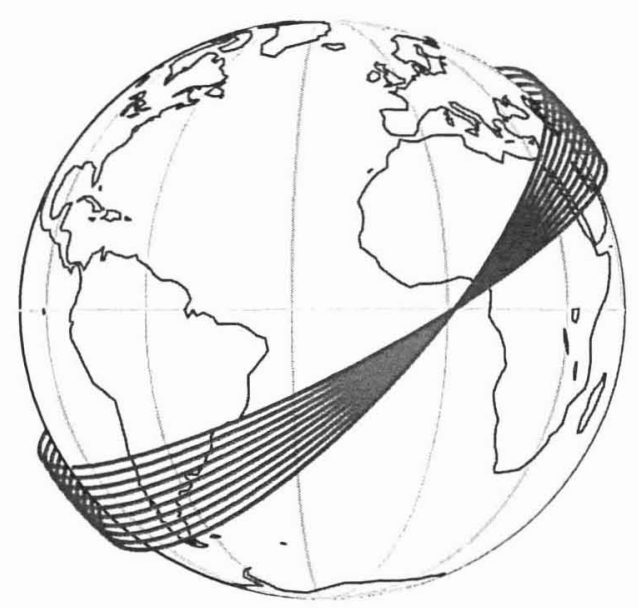




\section{MXER Tether Materials \& Fabrication Genealogy}

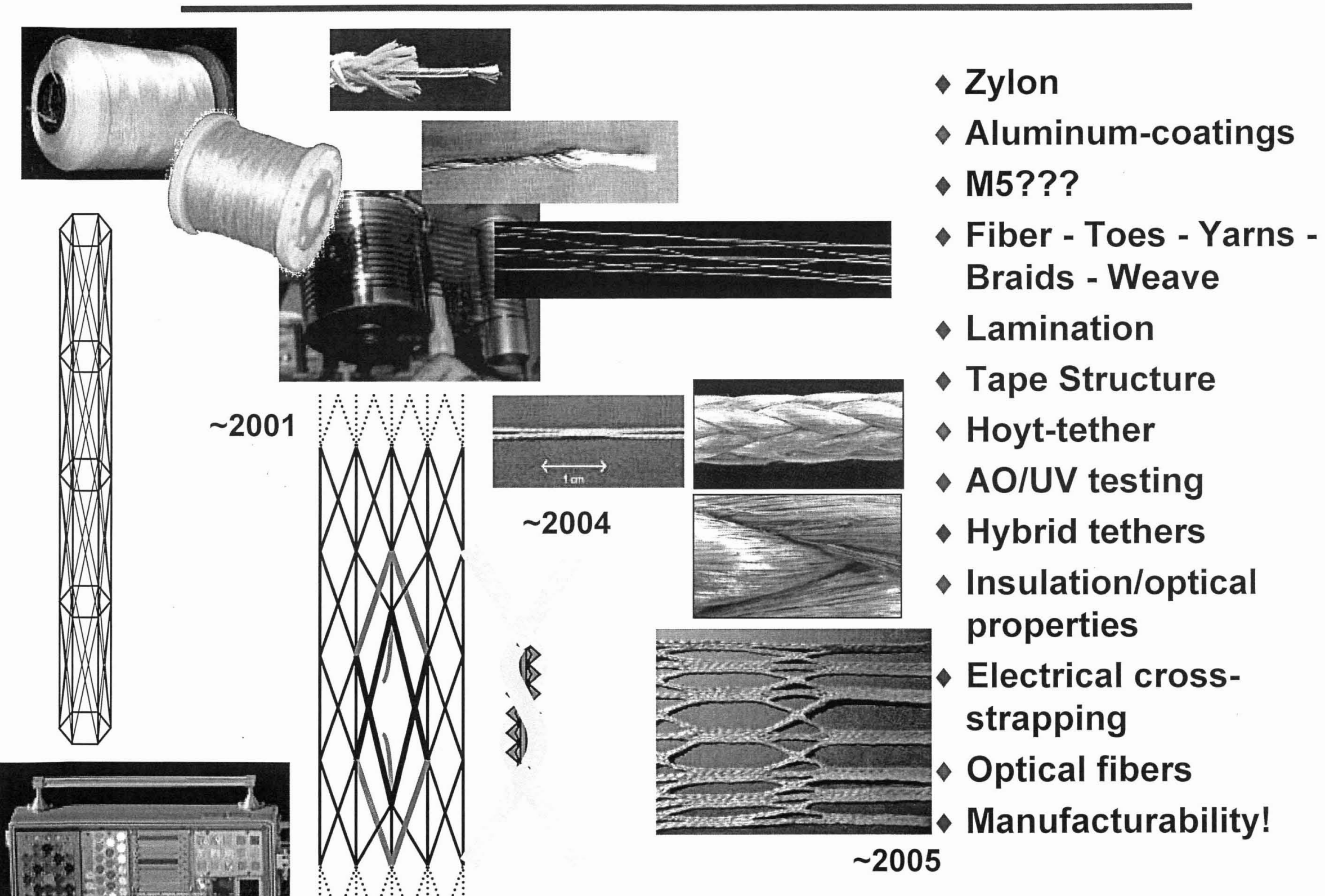

